Pacific Journal of Mathematics

NONSOLVABLE FINITE GROUPS ALL OF WHOSE LOCAL 


\title{
NONSOLVABLE FINITE GROUPS ALL OF WHOSE LOCAL SUBGROUPS ARE SOLVABLE, III
}

\author{
JoHN G. THOMPSON
}

In this paper, the simple $N$-groups for which $2 \notin \pi_{4}$ are classified. The proofs rely heavily on the fact that many subgroups of odd order are contained in just one maximal subgroup. The numbering of the sections is a continuation of II. ${ }^{1}$ The bibliographical references are to be found at the end of $I^{2}{ }^{2}$

10. Some uniqueness theorems. Throughout the remainder of this paper, \&5 denotes a simple $N$-group.

We set $\pi_{i}=\pi_{i}$ (S), $i=1,2,3,4$.

Lemmas 10.1 through 10.5 are proved on the hypothesis that $3 \in \pi_{3}$.

Let $\mathfrak{P}$ be a $S_{3}$-subgroup of $\mathbb{B S}$ and let $\mathbb{R}$ be a solvable subgroup of $\mathbb{S}$ which contains $\mathfrak{P}$. Let $\mathfrak{P}_{0}=\mathfrak{P} \cap \boldsymbol{O}_{3^{\prime}, 3}(\mathbb{R})$, and let $\mathfrak{X}$ be a normal elementary 3-subgroup of $\mathbb{R}$. Let

$\mathscr{A}(\mathfrak{R}, \mathfrak{P}, \mathfrak{X})=\{\mathfrak{A} \mid(1) \mathfrak{A}$ is a normal abelian subgroup of $\mathfrak{P}$.

(2) $\mathfrak{X} \subseteq \mathfrak{A} \subseteq \mathfrak{P}_{0}$.

(3) $m(\mathfrak{U})$ is maximal subject to (1) and (2).

(4) $|\mathfrak{A}|$ is maximal subject to (1), (2), (3).\}

An important property of $\mathscr{A}(\mathbb{S}, \mathfrak{P}, \mathfrak{X})$ is given by

LEMmA 10.1. If $\mathfrak{U} \in \mathscr{A}(\mathbb{R}, \mathfrak{P}, \mathfrak{X})$, then $\mathfrak{U} \in \mathscr{S} \mathscr{C} \mathscr{N}\left(\mathfrak{P}_{0}\right)$, where $\mathfrak{P}_{0}=\mathfrak{P} \cap \boldsymbol{O}_{3^{\prime}, 3}(\mathfrak{R})$.

Proof. Let $\mathfrak{A}^{*}=\boldsymbol{C}(\mathfrak{U}) \cap \mathfrak{P}_{0}$. Suppose $\mathfrak{A} \subset \mathfrak{A}^{*}$. Then there is a subgroup $\mathfrak{X}_{1}$ of $\mathfrak{U}^{*}$ such that $\mathfrak{A}_{1} \triangleleft \mathfrak{P},\left|\mathfrak{A}_{1}: \mathfrak{U}\right|=p$. Then $\mathfrak{N}_{1}$ is abelian and so (4) is violated.

Lemma 10.2. If $\mathfrak{A} \in \mathscr{A}(\mathfrak{Q}, \mathfrak{P}, \mathfrak{X})$, then $\boldsymbol{C}_{\mathbb{\Theta}}(\mathfrak{A})$ has a normal 3 complement.

Proof. Let $\mathfrak{D}$ be a $S_{3^{\prime}}$-subgroup of $\boldsymbol{C}_{\mathfrak{H}}(\mathfrak{U})$. Since $\mathfrak{A} \in \mathscr{S} \mathscr{C} \mathscr{N}\left(\mathfrak{P}_{0}\right)$, it follows that $\boldsymbol{Z}\left(\mathfrak{P}_{0}\right) \subseteq \mathfrak{A}$. Hence, $\boldsymbol{C}_{\Theta}(\mathfrak{U}) \subseteq \boldsymbol{N}_{\circledast}\left(\boldsymbol{Z}\left(\mathfrak{P}_{0}\right)\right)=\mathfrak{R}$, say. The

${ }^{1}$ Non solvable finite groups all of whose local subgroups are solvable, II, Pacific. J. Math., 33 (1970), 451-536.

2 Non solvable finite groups all of whose local subgroups are solvable, I, Bull. Amer. Math. Soc., 74 (1968), 383-437. 


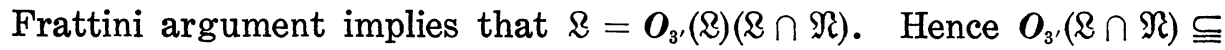
$\boldsymbol{O}_{3^{\prime}}(\mathbb{R})$, so that $\boldsymbol{O}_{3^{\prime}}(\mathbb{R} \cap \mathfrak{R})=\mathfrak{R} \cap \boldsymbol{O}_{3^{\prime}}(\mathbb{R})$, and $\mathbb{R} / \boldsymbol{O}_{3^{\prime}}(\mathbb{R})$ is incident with

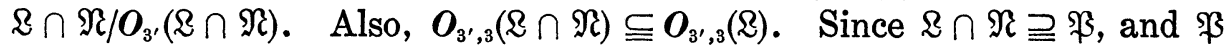
is a $S_{3}$-subgroup of $\mathfrak{R}$, it follows that $\boldsymbol{O}_{3^{\prime}, 3}(\mathfrak{R}) \cap \mathfrak{P} \subseteq \boldsymbol{O}_{3^{\prime}, 3}(\mathfrak{R} \cap \mathfrak{R})$. Hence, if we set $\mathfrak{P}_{1}=\boldsymbol{O}_{3^{\prime}, 3}(\mathfrak{N}) \cap \mathfrak{P}$, we get $\mathfrak{P}_{1} \subseteq \mathfrak{P}_{0}$. Since $\mathfrak{D}$ is a $3^{\prime}$-group contained in $C_{\oplus}(\mathfrak{R})$, we get $\mathfrak{A D}=\mathfrak{A} \times \mathfrak{D}$. Since $\mathfrak{P}_{1}$ and $\boldsymbol{O}_{3^{\prime}, 3}(\mathfrak{N}) / \boldsymbol{O}_{3^{\prime}}(\mathfrak{N})$ are incident, it follows that $C_{\Re_{1}}(\mathfrak{U}) \boldsymbol{O}_{3^{\prime}}(\mathfrak{R}) / \boldsymbol{O}_{3^{\prime}}(\mathfrak{R})$ is the set of fixed points of $\mathfrak{A}$ on $\boldsymbol{O}_{3^{\prime}, 3}(\mathfrak{R}) / O_{3^{\prime}}(\mathfrak{R})$. Since $\mathfrak{P}_{1} \subseteq \mathfrak{P}_{0}$, Lemma 10.1 implies that $C_{\mathfrak{P}_{1}}(\mathfrak{Q}) \subseteq$ भ. Hence, $\mathfrak{D}$ centralizes $\boldsymbol{O}_{3^{\prime}, 3}(\mathfrak{R}) / O_{3^{\prime}}(\mathfrak{N})$, by Lemma 3.7 of [20], so $\mathfrak{D} \subseteq O_{3^{\prime}}(\mathfrak{R})$. Since $\mathfrak{D}$ is a $S_{3^{\prime}}$-subgroup of $C_{\circledast}(\mathfrak{R})$, the lemma follows.

LemmA 10.3. Suppose $\mathfrak{A} \in \mathscr{A}(\mathbb{R}, \mathfrak{P}, \mathfrak{X})$, and $q$ is a prime $\neq 3$.

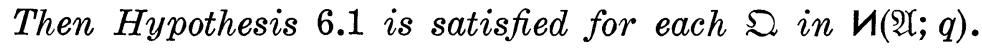

Proof. Since (8) is an $N$-group, the only condition which requires verification is (d). Suppose (d) does not hold. Let

$$
\begin{aligned}
& Q=\left\{\mathfrak{Q}_{0} \mid(1) \mathfrak{\Omega}_{0} \in \boldsymbol{h}(\mathfrak{H} ; q) .\right. \\
& \text { (2) There is a 3-solvable subgroup } \subseteq \text { of } \text { \& } \text { which } \\
& \text { contains } \left.\mathfrak{2} \mathfrak{\Omega}_{0} \text { such that } \mathfrak{\Omega}_{0} \nsubseteq \boldsymbol{O}_{3^{\prime}}(\mathfrak{S}) \text {. }\right\}
\end{aligned}
$$

Thus, $\mathscr{Q} \neq \varnothing$. Let $\mathfrak{Q}$ be a minimal element of $\mathscr{Q}$. Let

$$
\begin{aligned}
& \mathscr{S}=\left\{\mathfrak{S}_{0} \mid(1) \mathfrak{H} \cong \mathfrak{S}_{0} .\right. \\
& \text { (2) } \mathfrak{S}_{0} \text { is } 3 \text {-solvable. } \\
& \text { (3) } \cong \nsubseteq \boldsymbol{O}_{3^{\prime}}\left(\mathfrak{S}_{0}\right) \text {.\} }
\end{aligned}
$$

Thus, $\mathscr{S} \neq \varnothing$. Choose $\mathfrak{S}$ in $\mathscr{S}$ so that $\mathfrak{S} \cap \mathfrak{P}$ is maximal.

Let $\mathfrak{U}_{0}=C_{\mathfrak{r}}(\mathfrak{\Omega})$. By a basic property of $q$-groups, $\mathfrak{A}_{0}=C_{\mathfrak{U}}(\mathfrak{\Omega} / \boldsymbol{D}(\mathfrak{Q}))$. By the minimality of $\mathfrak{Q}, \mathfrak{X}$ acts irreducibly on $\mathfrak{Q} / \boldsymbol{D}(\mathfrak{Z})$. As $\mathfrak{U}$ is abelian, it follows that $\mathfrak{A} / \mathfrak{A}_{0}$ is cyclic.

Let $\mathfrak{S}_{3}$ be a $S_{3}$-subgroup of $\mathfrak{S}$ which contains $\mathfrak{A}$, and set $\widetilde{\mathfrak{S}}_{3}=$ $\mathfrak{S}_{3} \cap \boldsymbol{O}_{3^{\prime}, 3}(\mathfrak{S})$. Let $\mathfrak{B}=C\left(\mathfrak{H}_{0}\right) \cap \widetilde{\mathfrak{S}}_{3^{\prime}}$. Thus, $\mathfrak{B} \boldsymbol{O}_{3^{\prime}}(\mathfrak{S}) / \boldsymbol{O}_{3^{\prime}}(\mathfrak{S})=C_{V}\left(\mathfrak{H}_{0}\right)$, where $V=\boldsymbol{O}_{3^{\prime}, 3}(\mathfrak{S}) / O_{3^{\prime}}(\mathfrak{S})$. By Lemma 3.7 of [20], $\mathfrak{Q}$ does not centralize $\boldsymbol{C}_{V}\left(\mathfrak{\Re}_{0}\right)$,

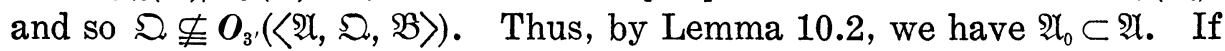
$\mathfrak{A}_{1}$ is any subgroup of $\mathfrak{A}$ which is not contained in $\mathfrak{A}_{0}$, the irreducibility of $\mathfrak{A}$ on $\mathfrak{Q} / \boldsymbol{D}(\mathfrak{Q})$ forces $\mathfrak{Q}=\left[\mathfrak{\Omega}, \mathfrak{A}_{1}\right]$.

Suppose $\mathfrak{A}_{0}$ contains an element $Z$ of $Z(\mathfrak{P})^{\sharp}$. Then $\langle\mathfrak{A}, \mathfrak{\Omega}, \mathfrak{B}\rangle \leqq C(Z)$, so that $C(Z) \in \mathscr{S}$. By our choice of $\mathfrak{S}$, we get $\mathfrak{P} \subseteq \mathfrak{S}$. Hence, [V, $\mathfrak{R}]$ is covered by $\mathfrak{A}$, since $\mathfrak{A} \triangleleft \mathfrak{P}$ and $V$ is covered by $\mathfrak{P}$. Hence $[V, \mathfrak{A}, \mathfrak{Q}]$ is covered by $[\mathfrak{A}, \mathfrak{Q}]=\mathfrak{Q}$, so that $[V, \mathfrak{A}, \mathfrak{Q}]=1$. This violates Lemma 5.16 so no such $Z$ is available.

Since $\mathfrak{A}$ is noncyclic, and since $\mathfrak{A} \supseteqq \boldsymbol{Z}\left(\mathfrak{P}_{0}\right) \supseteqq Z(\mathfrak{P})$, it follows that $\mathfrak{A}$ contains an element $\mathfrak{u}$ of $\mathscr{U}(\mathfrak{P})$. Let $\mathfrak{u}_{0}=\mathfrak{u} \cap \mathfrak{A}_{0}$, so that $\left|\mathfrak{U}_{0}\right|=3$, $\mathfrak{U}_{0} \nsubseteq \boldsymbol{Z}(\mathfrak{P})$. Then $\langle\mathfrak{A}, \mathfrak{Q}, \mathfrak{B}\rangle \subseteq \boldsymbol{C}_{\oplus}\left(\mathfrak{U}_{0}\right)$, so that 


$$
\boldsymbol{C}_{\circledast}\left(\mathfrak{U}_{0}\right) \in \mathscr{S} .
$$

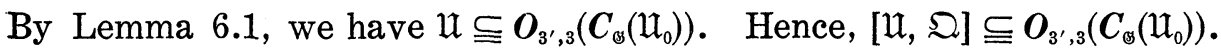
But $\mathfrak{u} \nsubseteq \mathfrak{U}_{0}$, so $[\mathfrak{u}, \mathfrak{Q}]=\Omega$. Hence, $\mathfrak{\varrho} \subseteq \boldsymbol{O}_{3^{\prime}, 3}\left(C_{\mathfrak{\Theta}}\left(\mathfrak{U}_{0}\right)\right)$, which gives $\mathfrak{\varrho}$ $\boldsymbol{O}_{3^{\prime}}\left(\boldsymbol{C}_{\Theta}\left(\mathfrak{U}_{0}\right)\right)$, against $(10.2)$. This contradiction completes the proof.

Lemma 10.4. Suppose $\mathfrak{A} \in \mathscr{A}(\mathfrak{R}, \mathfrak{P}, \mathfrak{X})$ and $m(\mathfrak{A}) \leqq 2$. Then $\mathfrak{P}_{0}=$ $\mathfrak{P} \cap \boldsymbol{O}_{3^{\prime}, 3}(\mathfrak{Q})$ is the central product of a cyclic group and a nonabelian group of order $3^{3}$ and exponent 3 . Furthermore, $\mathfrak{P}=\mathfrak{B}_{0}\langle P\rangle$, where $P$ is an element of $\mathfrak{B}$ of order 3 which lies in an abelian normal subgroup of $\mathfrak{P}$.

Proof. Since $m(\mathfrak{U}) \leqq 2$, so also $m(\mathfrak{X}) \leqq 2$. Let $\mathfrak{P}_{1}=\boldsymbol{C}_{\mathfrak{P}_{0}}(\mathfrak{X})$ so that $\mathfrak{P}_{1} \triangleleft \mathfrak{P},\left|\mathfrak{P}_{0}: \mathfrak{P}_{1}\right| \leqq 3$. Choose $\mathfrak{B}_{0}$ in $\mathscr{B}\left(\mathfrak{P}_{1}\right)^{3}$ and let $\mathfrak{B}=\Omega_{1}\left(\mathfrak{B}_{0}\right)$. Let $\mathfrak{C}$ be a normal abelian subgroup of $\mathfrak{P}$ of largest order subject to $\mathfrak{E} \subseteq \mathfrak{B}$. Then $\mathfrak{E} \in \mathscr{S} \mathscr{C} \mathscr{N}(\mathfrak{B})$ and $\mathfrak{X} \subseteq \mathfrak{C}$, so that $m(\mathfrak{E}) \leqq 2$. Hence, $\mathfrak{B}$, being of exponent 3 and class $\leqq 2$, is either elementary of order 9 or nonabelian of order $3^{3}$.

Let $\mathfrak{R}=\boldsymbol{N}_{\mathfrak{\varepsilon}}\left(\mathfrak{P}_{0}\right)$. Thus, $\boldsymbol{O}_{\mathfrak{3}^{\prime}, 3}(\mathfrak{N})=\mathfrak{P}_{0} \times\left(\mathfrak{R} \cap \boldsymbol{O}_{3^{\prime}}(\mathfrak{R})\right)$. Since $\mathfrak{B}_{0} \in$ $\mathscr{B}\left(\mathfrak{P}_{1}\right)$ it follows that a $S_{3^{\prime}}$-subgroup of $\mathfrak{R} / \mathfrak{R} \cap O_{3^{\prime}}(\mathbb{R})$ is faithfully represented on $\mathfrak{B}_{0}$, so, by 0.3 .6 , is faithfully represented on $\mathfrak{B}$. Hence $\boldsymbol{C}_{\mathfrak{R}}(\mathfrak{B}) \subseteq\left(\mathfrak{N} \cap \boldsymbol{O}_{3^{\prime}}(\mathfrak{R})\right) \cdot \mathfrak{P}$. Since $\boldsymbol{C}_{\mathfrak{R}}(\mathfrak{B}) \triangleleft \mathfrak{N}$, we have $\boldsymbol{C}_{\mathfrak{R}}(\mathfrak{B}) \cong \mathfrak{P}_{0} \times(\mathfrak{R} \cap$ $\boldsymbol{O}_{3^{\prime}}(\mathfrak{R})$ ). In particular, $C_{\mathfrak{\beta}}(\mathfrak{B}) \subseteq \mathfrak{P}_{0}$.

If $\mathfrak{B}$ is elementary of order 9 , then by Lemma $0.8 .9, \mathfrak{B}$ is contained in an elementary normal subgroup $\mathfrak{B}^{*}$ of $\mathfrak{P}$ of order $3^{3}$. Hence $\mathfrak{B}^{*} \subseteq$ $\mathfrak{F}_{0}$. But this violates $(3)$ in the definition of $\mathscr{A}(\mathfrak{R}, \mathfrak{P}, \mathfrak{X})$. Hence, $\mathfrak{B}$ is nonabelian of order $3^{3}$ and exponent 3 , and $(5$ is of order 9.

Since $\mathfrak{B}_{0} \in \mathscr{B}\left(\mathfrak{P}_{1}\right)$, it follows that $\mathfrak{P}_{1}=\mathfrak{B} \cdot C_{\mathfrak{p}_{1}}(\mathfrak{B})$. Since $\mathfrak{X}$ is contained in $Z(\mathfrak{B})$, we have $|\mathfrak{X}| \leqq 3$, and so $\mathfrak{P}_{0}=\mathfrak{P}_{1}$. Since $\mathfrak{B}$ and $\mathfrak{F}_{1}$ are normal in $\mathfrak{P}$, so also $\boldsymbol{C}_{\mathfrak{\Re}_{1}}(\mathfrak{B}) \triangleleft \mathfrak{P}$. If $\boldsymbol{C}_{\mathfrak{P}_{0}}(\mathfrak{B})$ is noncyclic, then there is an elementary subgroup $\mathbb{F}$ of $C_{\mathfrak{q}_{0}}(\mathfrak{B})$ of order 9 with $₫ \triangleleft \mathfrak{F}$. But then $\left\langle\mathfrak{F}\right.$, (ㄷ) $\triangleleft \mathfrak{P}$, is elementary of order $3^{3}$, against $m(\mathfrak{A}) \leqq 2$. We conclude that $\boldsymbol{C}_{\mathfrak{\Re}_{0}}(\mathfrak{B})$ is cyclic, so that the first assertion of the lemma holds.

Since $3 \in \pi_{3}$, $\mathfrak{P}$ contains a normal elementary subgroup $\mathfrak{Y}$ of order $3^{3}$. Thus $\mathfrak{P}_{0} \cap \mathfrak{Y}$ is of order 9 , so we can choose $P \in \mathfrak{Y}-\mathfrak{B}_{0}$. Since $\boldsymbol{N}_{\varepsilon}\left(\mathfrak{P}_{0}\right) / \mathfrak{P}_{0} \boldsymbol{C}_{\varepsilon}\left(\mathfrak{P}_{0}\right)$ is faithfully represented on $\mathfrak{B} / D(\mathfrak{B})$, we have $\mathfrak{P}=\mathfrak{P}_{0}\langle P\rangle$. The proof is complete.

LemmA 10.5. Suppose $\mathfrak{A} \in \mathscr{A}(\mathfrak{S}, \mathfrak{P}, \mathfrak{X})$ and $m(\mathfrak{X}) \leqq 2$. Let $q$ be any prime $\neq 3$, and let $\mathfrak{Q}$ be a maximal element of $И(\mathfrak{P} ; q)$. Then $\mathfrak{P} \subseteq N_{\circledast}(\mathfrak{D})^{\prime}$.

Proof. Let $\&$ be any element of $\mathscr{S} \mathscr{C} \mathscr{N}_{3}(\mathfrak{P})$. By the transitivity

${ }^{3}$ See Definition 26 and Lemma 5.18. 
theorem, $N(\mathbb{F})=(N(\mathbb{F}) \cap N(\mathfrak{Q})) O_{3^{\prime}}(N(\mathbb{F}))$. Suppose we have shown that $\mathfrak{P} \subseteq N(\mathfrak{F})^{\prime}$. Then $\mathfrak{P} \subseteq(N(\mathfrak{F}) \cap N(\mathfrak{Q}))^{\prime}$, so all the more so, $\mathfrak{P} \subseteq N(\mathfrak{Q})^{\prime}$.

Since $m(\mathfrak{X}) \leqq 2$ for some $\mathfrak{A} \in \mathscr{A}(\mathfrak{R}, \mathfrak{P}, \mathfrak{X})$, the structure of $\mathfrak{P}$ is given by Lemma 10.4. It is straightforward to verify that $\&$ is the only subgroup of $\mathfrak{P}$ of its isomorphism class; in particular, $\mathbb{F}$ is weakly closed in $\mathfrak{P}$. By a standard transfer theorem [20, p. 212], we get $\mathfrak{P} \subseteq N(\mathfrak{F})^{\prime}$, and we are done.

Theorem 10.1. If $p \in \pi_{3}-\{2\}, q \in \pi(\mathbb{S})-\{p\}$, $\mathfrak{P}$ is a $S_{p}$-subgroup of $\mathbb{B S}$ and $\mathfrak{Q}$ is a maximal element of $И(\mathfrak{B} ; q)$, then $\mathfrak{P} \subseteq N_{\Theta}(\mathfrak{Q})^{\prime}$.

Proof. First, suppose $p \geqq 5$. Let $\mathfrak{S}$ be a $p$-solvable subgroup of $\&$ with $S_{p}$-subgroup $\mathfrak{K}_{p}$. Choose $\Re \in \mathscr{S} \mathscr{C} \mathscr{N}\left(\mathfrak{K}_{p}\right)$. Since $\left[\mathfrak{K}_{p}, \Re, \Re\right]=1$ it follows from $(B)$ that $\Re \subseteq O_{p^{\prime}, p}(\mathfrak{F})$. Choose $\mathfrak{A} \in \mathscr{S} \mathscr{C} \mathscr{N}_{3}(\mathfrak{P})$, and let $\mathscr{F}=\{\mathfrak{A}\}$. Then Hypothesis 6.2 is satisfied with this choice of $\mathfrak{A}, \mathscr{F}$. Conditions (a), (b), (c) of Hypothesis 6.1 are also satisfied, since $\mathbb{B}$ is an $N$-group. Hence, Hypothesis 6.1 is satisfied, by Lemma 6.3. We have already shown that condition (f) of Hypothesis 6.3 holds, so Hypothesis 6.3 holds. Thus, the theorem follows from Corollary 6.2.

We may therefore assume that $p=3$. If 15 contains a 3 -solvable subgroup $\mathbb{R}$ such that $\mathbb{Q} \supseteqq \mathfrak{P}$, and such that for some normal elementary 3 -subgroup $\mathfrak{X}$ of $\mathbb{R}$, there is $\mathfrak{A}$ in $\mathscr{A}(\mathbb{R}, \mathfrak{P}, \mathfrak{X})$ with $m(\mathfrak{H}) \leqq 2$, we are done by Lemma 10.5. Hence we may assume that

$$
m(\mathfrak{U}) \geqq 3 \text { for all } \mathfrak{A} \in \mathscr{A}(\mathfrak{R}, \mathfrak{P}, \mathfrak{X}) \text { and all relevant } \mathfrak{R}, \mathfrak{X} \text {. }
$$

Set $\mathfrak{L}=N_{\mathscr{\Theta}}(\mathfrak{Q})$. If $\mathfrak{Q}=1$, we are done, so suppose $\mathfrak{Q} \neq 1$. Then $\mathfrak{Z}$ is solvable and $\mathfrak{P} \subseteq \mathbb{R}$. Choose $\mathfrak{A} \in \mathscr{A}(\mathbb{R}, \mathfrak{P}, \mathfrak{X})$. By Lemma 6.2, the application of which is guaranteed by Lemma 10.3, we get

$$
\left\{\mathfrak{Q}^{G} \mid G \in \mathbb{S}, \mathfrak{Q}^{G} \subseteq \mathfrak{P}\right\}=\left\{\mathfrak{U}^{L} \mid L \in \mathfrak{R}, \mathfrak{Q}^{L} \subseteq \mathfrak{P}\right\} \text {. }
$$

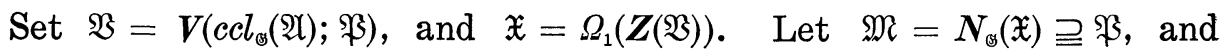
choose $\mathfrak{B} \in \mathscr{A}(\mathfrak{M}, \mathfrak{P}, \mathfrak{X})$. By Lemma 6.2, with $\mathfrak{B}$ in the role of $\mathfrak{A}$, we get $\mathfrak{M}=\boldsymbol{O}_{3^{\prime}}(\mathfrak{M})(\mathfrak{M} \cap \mathbb{R})$.

Suppose $G \in \mathbb{S}$ and $\mathfrak{A}^{G} \subseteq \mathfrak{M}$. We will show that $G \in \mathfrak{M}$. We may choose $M_{1}$ in $\mathfrak{M}$ such that $\mathfrak{A}^{G M_{1}} \subseteq \mathfrak{P}$. Let $G_{1}=G M_{1}$. Since $\mathfrak{A}^{G_{1}} \subseteq \mathfrak{P}, \mathfrak{A}$ normalizes $\mathfrak{\Omega}_{1}^{G_{1}^{-1}}$, so $\mathfrak{\Omega}_{1}^{G_{1}^{-}}=\mathfrak{Q}^{C}$ for some $C \in \boldsymbol{C}(\mathfrak{A})$. Since $\mathfrak{X} \subseteq \mathfrak{A}$, we get $C \in \mathfrak{M}$. Let $G_{2}=C G_{1}$, so that $\mathfrak{\Omega}=\mathfrak{\Omega}^{G_{2}}$.

Since $\mathfrak{B} \subseteq O_{3^{\prime}, 3}(\mathbb{R})$, we have $\mathbb{R}=\boldsymbol{O}_{3^{\prime}}(\mathbb{R})(\mathbb{R} \cap \mathfrak{M})=\boldsymbol{O}_{3^{\prime}}(\mathbb{R})(\mathbb{R} \cap N(\mathfrak{B}))$. Hence, $G_{2}=L_{2} L_{1}$ where $L_{1} \in \mathbb{R} \cap N(\mathfrak{B}), L_{2} \in \boldsymbol{O}_{3^{\prime}}(\mathbb{R})$. Since $\mathfrak{X}^{G_{1}} \subseteq \mathfrak{P}$, so also $\mathfrak{A}^{G_{2}} \subseteq \mathfrak{P}$, since $\mathfrak{A}^{G_{2}}=\mathfrak{A}^{G_{1}}$. Since $L_{1} \in N(\mathfrak{B})$, and $\mathfrak{A} \subseteq \mathfrak{B}$, we have $\mathfrak{P}^{L_{1}} \subseteq \mathfrak{P}$. Thus, for each $A$ in $\mathfrak{A}, A^{-L_{1}} \cdot A^{L_{2} L_{1}} \in \mathfrak{P}$. Since $A^{-L_{1}} \cdot A^{L_{2} L_{1}}=$ $\left[A, L_{2}\right]^{L_{1}}$, we get that $\left[A, L_{2}\right]$ is a 3 -element. Since $L_{2} \in O_{3^{\prime}}(\mathbb{R})$, we get $\left[A, L_{2}\right]=1$. Hence, $L_{2} \in C_{\mathfrak{\Theta}}(\mathfrak{R}) \subseteq \mathfrak{M}$. Since $L_{1} \in N(\mathfrak{B}) \subseteq \mathfrak{M}$, we get 
$G_{2} \in \mathfrak{M}$. Since $C \in C(\mathfrak{U}) \subseteq \mathfrak{M}$, we get $G_{1} \in \mathfrak{M}$. Hence, $G \in \mathfrak{M}$.

We may now apply the argument of Lemma 0.17.2 to complete a proof that $\mathfrak{M}=\boldsymbol{O}^{3}(\mathfrak{M})$. Since $\mathfrak{M}=\boldsymbol{O}_{3^{\prime}}(\mathfrak{M})(\mathbb{R} \cap \mathfrak{M})$, we also have $\mathfrak{L}=\boldsymbol{O}^{3}(\mathfrak{R})$. The proof is complete.

We next turn briefly to $\pi_{4}$.

TheOREM 10.2. If $p \in \pi_{4}$ and $p \geqq 5$, then $\mathscr{A}_{4}(p) \subseteq \mathscr{C}^{*}(G)$.

Proof. Let $\mathfrak{B}$ be a $S_{p}$-subgroup of $\mathbb{S}$ and let $\mathfrak{X}=Z(J(\mathfrak{P}))$, $\mathfrak{M}=$ $N(\mathfrak{X})$. By a result of Glauberman [15], if $\subseteq$ is any solvable subgroup of $\mathbb{S}$ which contains $\mathfrak{P}$, then $\mathfrak{S} \subseteq \mathfrak{M}$. Hence, $\mathfrak{P} \in \mathscr{A}^{*}(\mathfrak{S})$.

Let $\mathscr{A}_{1}^{*}(\mathfrak{P})=\{\mathfrak{A} \mid \mathfrak{A}$ is a subgroup of $\mathfrak{P}, \mathfrak{A}$ contains some element of $\mathscr{S C C N}_{3}\left(\mathfrak{P}^{M}\right)$ for some $M$ in $\left.\mathfrak{M}.\right\}$

Suppose $\mathscr{A}_{1}^{*}(\mathfrak{P}) \nsubseteq \mathscr{C}^{*}(\mathbb{S})$. Let $\mathfrak{A}$ be an element of largest order in $\mathscr{A}_{1}^{*}(\mathfrak{B})-\mathscr{C}^{*}(G)$, and let $\subseteq$ be a solvable subgroup of $\mathbb{S}$ which contains $\mathfrak{A}$ and satisfies $\subseteq \nsubseteq \mathfrak{M}$. Choose $M$ in $\mathfrak{M}$ and $\left(\mathfrak{F}\right.$ in $\mathscr{S P C}_{\mathfrak{N}}\left(\mathfrak{P}^{M}\right)$, such that $\mathfrak{F} \subseteq \mathfrak{A}$.

The maximality of $\mathfrak{A}$ guarantees that $\mathfrak{A}$ is a $S_{p}$-subgroup of $\mathfrak{S}$. Since $\left(5 \subseteq \mathfrak{S}\right.$, it follows that $\boldsymbol{O}_{p^{\prime}}(\mathfrak{S})=1$, by Theorem 6.1. Hence $\mathfrak{S} \subseteq$ $N(Z(J(\mathfrak{A})))$, by a result of Glauberman. Since $N_{\mathfrak{\beta}}(Z(J(\mathfrak{A}))) \supset \mathfrak{A}$, we get $\subseteq \subseteq \mathfrak{M}$, against our choice of $\subseteq$. Hence, $\mathscr{A}_{1}^{*}(\mathfrak{P}) \subseteq \mathscr{L}^{*}(\mathbb{S})$.

We may now complete the proof by using the argument of Theorem 0.24.4.

Theorem 10.3. Suppose $p \in \pi_{4}, p \geqq 5$, $\mathfrak{S}$ is a $S_{p}$-subgroup of (S), and $\mathfrak{M}=\boldsymbol{M}(\mathfrak{P})$. Let $\mathfrak{I}$ be a $S_{2}$-subgroup of $\mathfrak{M}$ permutable with $\mathfrak{P}$. Then one of the following holds:

(a) $2 \in \pi_{2}$.

(b) For each $T$ in $\mathfrak{I}^{\sharp}, C_{\mathfrak{m}}(T)$ has cyclic $S_{p}$-subgroups and $S_{2, p^{-}}$ subgroups of $\mathfrak{M}$ are p-closed.

(c) $\mathfrak{I}$ is a $S_{2}$-subgroup of (S).

Proof. Let $\mathfrak{P}_{0}=\boldsymbol{O}_{p}(\mathfrak{P} \mathfrak{T})$. Since $p \in \pi_{4}, \mathfrak{I}$ is faithfully represented on $\mathfrak{P}_{0}$. Suppose (a), (b), (c) fail. Let $\mathfrak{I}^{*}$ be a $S_{2}$-subgroup of $\mathbb{B}$ which contains $\mathfrak{I}$. Suppose $\boldsymbol{C}_{\mathfrak{\beta}_{0}}(T)$ is noncyclic for some involution $T$ of $\mathfrak{I}$. Then $\boldsymbol{C}_{\mathfrak{F}_{0}}(T) \in \mathscr{M}_{4}(\mathfrak{P})$, so $\boldsymbol{C}_{\mathfrak{G}}(T) \subseteq \mathfrak{M}$. Since $\mathscr{S}^{-} \mathscr{N}_{3}\left(\mathfrak{T}^{*}\right) \neq \varnothing$, it follows that $\boldsymbol{C}_{\mathbb{\Theta}}(T)$ contains an elementary subgroup of order 8, so $\mathfrak{I}$ contains an elementary subgroup $\mathfrak{X}$ of order 8 . Let

$$
\widehat{\mathfrak{A}}=\left\{A \mid A \in \mathfrak{U}^{\sharp}, C_{\mathfrak{F}_{0}}(A) \text { is noncyclic }\right\} \text {. }
$$

Suppose $\mathfrak{I}$ contains an elementary subgroup of order 16 . Then there is a four-subgroup $\mathfrak{B}$ of $\mathfrak{I}$ such that $\boldsymbol{C}_{\mathfrak{B}}(\mathfrak{B})$ is noncyclic. Hence $\boldsymbol{C}_{\mathfrak{B}}(B) \subseteq$ 
$\mathfrak{M}$ for all $B$ in $\mathfrak{B}^{\sharp}$. Thus, if $N \in N_{\circledast}(\mathfrak{T})$, then $\mathfrak{P}_{0}^{N}$ is normalized by $\mathfrak{B}$,

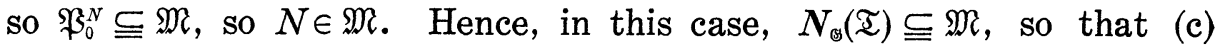
holds. We may assume that $\mathfrak{I}$ contains no elementary subgroup of order 16. Let $₹$ be a normal elementary subgroup of $\mathfrak{I}^{*}$ of order 8 , and let $\mathfrak{F} \in \mathscr{S} \mathscr{C} \mathscr{N}\left(\mathfrak{T}^{*}\right)$, $₹ \subseteq \mathfrak{F}$. Since $C_{\circledast}(T) \subseteq \mathfrak{M}$, so also $C_{\mathscr{\uplus}}(T) \subseteq \mathfrak{M}$. If $\mathfrak{F} \subseteq \mathfrak{M}$, then we may take $T \in \mathfrak{F}$, so that $\mathfrak{F} \subseteq \mathfrak{M}$. But then $\mathfrak{P}_{0} \in$ $\boldsymbol{U}_{\mathscr{\Theta}}(\mathfrak{F} ; p)$, so $N_{\mathscr{\Theta}}\left(\mathfrak{B}_{0}\right)$ contains a $S_{2}$-subgroup of (S), by the transitivity theorem. This is not the case, so $C_{\mathfrak{G}}(T)=\mathfrak{F}_{0}$ is of order 4. We may now take $\mathfrak{A}=\langle T\rangle \times \mathfrak{F}_{0}$. Then $\hat{\mathfrak{A}} \cap \mathfrak{F}_{0}=\varnothing$. Choose $N$ in $N_{\mathfrak{x}^{*}}(\mathfrak{T})-\mathfrak{T}$. Then $\mathfrak{I}$ normalizes $\mathfrak{P}_{0}^{N}$, so $\hat{\mathfrak{A}}^{N} \cap \mathfrak{F}_{0}=\varnothing$. But we also have $\hat{\mathfrak{U}}^{N} \cap \hat{\mathfrak{A}}=\varnothing$, since $N \notin \mathfrak{M}$. Thus, $|\hat{\mathfrak{A}}| \leqq 2$. Since $O_{2}(\mathfrak{P T})=1$, Lemma 5.3 yields an $\mathfrak{A}$-subgroup $\mathfrak{P}_{1}$ of $\mathfrak{P}_{0}$ which is the direct product of $3 \mathfrak{A}$-subgroups of order $p, \mathfrak{P}_{1}=\mathfrak{P}_{11} \times \mathfrak{P}_{12} \times \mathfrak{P}_{13}$, such that $\boldsymbol{C}_{\mathfrak{4}}\left(\mathfrak{P}_{1}\right)=1$. Let $\mathfrak{A}_{i}=C_{21}\left(\mathfrak{P}_{1 i}\right)$, $\mathfrak{A}_{i j}=\mathfrak{A}_{i} \cap \mathfrak{A}_{\jmath}$. Since $|\hat{\mathfrak{A}}| \leqq 2$, we may assume that $\mathfrak{A}_{12}=\mathfrak{A}_{23}=\langle A\rangle$. But then $A$ centralizes $\mathfrak{P}_{1}$ contrary to construction. Thus, $\mathfrak{I}$ is a $S_{2}$-subgroup of (S) in this case.

We may now assume that $\boldsymbol{C}_{\mathfrak{\Re}_{0}}(T)$ is cyclic for all $T$ in $\mathfrak{I}^{*}$. Hence, $\mathfrak{I}$ contains no elementary subgroup of order 8 . If $\mathfrak{P}_{0}=\mathfrak{P}$, then $(b)$ holds. We may assume that $\mathfrak{P}_{0} \subset \mathfrak{P}$.

Let $\mathfrak{I}_{0}=\mathfrak{I} \cap \boldsymbol{O}_{p, 2}(\mathfrak{P T}), \mathfrak{P}_{1}^{*}=\boldsymbol{N}_{\mathfrak{B}}\left(\mathfrak{I}_{0}\right), \mathfrak{I}_{0}^{*}=\left[\mathfrak{I}_{0}, \mathfrak{P}_{1}^{*}\right]$. Since $p \geqq 5$, $\mathfrak{P}_{1}^{*}$ centralizes every characteristic abelian subgroup of $\mathfrak{I}_{0}^{*}$. Hence $\mathfrak{I}_{0}^{*}$ is special, by Lemma 5.17. Suppose $\left|\boldsymbol{Z}\left(\mathfrak{I}_{0}^{*}\right)\right| \geqq 4$. Let $J$ be any involution of $\boldsymbol{Z}\left(\mathfrak{T}_{0}^{*}\right)$. Since $\boldsymbol{C}_{\mathfrak{F}_{0}}(J)$ is cyclic, $\mathfrak{I}_{0}^{* \prime}$ centralizes $\boldsymbol{C}_{\mathfrak{F}_{0}}(J)$. Since $\left|\boldsymbol{Z}\left(\mathfrak{I}_{0}^{*}\right)\right| \geqq 4$, it follows that $\mathfrak{I}_{0}^{* \prime}=\boldsymbol{Z}\left(\mathfrak{I}_{0}^{*}\right)$ centralizes $\mathfrak{P}_{0}$. This is not the case, since $\mathfrak{B}_{0}$ is noncyclic. Hence, $\mathfrak{I}_{0}^{*}$ is extra special of width 2 and $p=5$. It follows that if $J$ is any noncentral involution of $\mathfrak{I}_{0}^{*}$, then $C_{\mathfrak{F}_{0}}(J)$ is noncyclic. This is not the case. The proof is complete.

Hypothesis 10.1. ALS (S) contains an element $\mathfrak{M}$ with the properties:

(i ) $|\mathfrak{M}|$ is even.

(ii) $C_{\leftrightarrow}(I) \subseteq \mathfrak{M}$ for every involution $I$ of $\mathfrak{M}$.

(iii) If $\mathfrak{I}$ is a $S_{2}$-subgroup of $\mathfrak{M}$, then $N_{\Theta}(\mathfrak{I}) \subseteq \mathfrak{M}$.

(iv) If $p$ is a prime such that some element of $\mathfrak{M}$ of order $p$ is a product of two involutions of $\& 3$ and is not a product of two involutions of $\mathfrak{M}$, then $S_{p}$-subgroups of $\mathfrak{M}$ are cyclic.

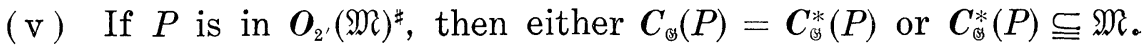

THEOREM 10.4. Under Hypothesis 10.1, (5) is isomorphic to $L_{2}\left(2^{n}\right)$ or $S z\left(2^{n}\right)$ for some $n .^{4}$

Proof. By Lemma 5.38, $i((\mathfrak{S})=i(\mathfrak{M})=1$.

${ }^{4}$ This theorem is an immediate consequenee of Bender's theorem on strongly embedded subgroups. 
We next show that if $\mathscr{S}$ is any element of $\mathscr{S O L} \mathscr{L}(\mathbb{S})$ such that $\mathfrak{S} \cap \mathfrak{M}$ contains a four-group, then $\mathfrak{S} \subseteq \mathfrak{M}$. Let $I_{0}$ be an involution of $\mathfrak{S} \cap \mathfrak{M}$ and let $\mathfrak{I}_{0}$ be a $S_{2}$-subgroup of $\mathfrak{F}$ containing $I_{0}$. Two applications of Hypothesis 10.1(ii) yield $\mathfrak{I}_{0} \subseteq \mathfrak{M}$. Let $\mathfrak{B}$ be a foursubgroup of $\mathfrak{I}_{0}$. Then $\boldsymbol{O}_{2^{\prime}}\left(\mathfrak{S}_{\mathcal{E}}\right)$ is generated by its subgroups $\boldsymbol{O}_{2^{\prime}}(\mathfrak{K}) \cap$ $\boldsymbol{C}_{\mathfrak{B}}(V), V \in \mathfrak{B}^{\sharp}$, so $\boldsymbol{O}_{2^{\prime}}(\mathfrak{S}) \subseteq \mathfrak{M}$. Let $\mathfrak{I}_{1}=\boldsymbol{O}_{2^{\prime}, 2}\left(\mathfrak{S}_{\mathcal{E}}\right) \cap \mathfrak{I}_{0}$, so that

$$
\mathfrak{S}=O_{2^{\prime}}(\mathfrak{K}) N_{\mathfrak{g}}\left(\mathfrak{I}_{1}\right) \text {. }
$$

If $N \in N_{\mathfrak{g}}\left(\mathfrak{I}_{1}\right)$ and $T$ is an involution of $\mathfrak{I}_{1}$, then $T^{N} \in \mathfrak{I}_{1}$, so that $T^{N}=T^{M}$ for some $M$ in $\mathfrak{M}$ and $N M^{-1} \in C_{\Theta}(T) \subseteq \mathfrak{M}$, so $N \in \mathfrak{M}$. Hence, $\mathfrak{S} \subseteq \mathfrak{M}$.

Let $I$ be an involution of $\mathfrak{M}$ and let $\mathbb{E}=C_{\mathfrak{G}}(I)=C_{\mathfrak{n}}(I)$. Let $m=|\mathfrak{M}: \mathfrak{C}|$, the number of involutions of $\mathfrak{M}$. Choose $G$ in $\mathbb{S}-\mathfrak{M}$. Then $\mathfrak{M G}$ contains exactly $m$ involutions $T_{1}, \cdots, T_{m}$, and

$$
\left\{1, T_{1} T_{2}, \cdots, T_{1} T_{m}\right\}
$$

is a set of representatives for the cosets of $\mathbb{S}$ in $\mathfrak{M}$. Holding $G$ fixed and letting $I$ range over the involutions of $\mathfrak{M}$, it follows that $T_{1} T_{i}$ commutes with no involution of $\mathfrak{M}, 2 \leqq i \leqq m$. By the results of [9], $m \geqq 3$.

Let $\mathfrak{S}=\left\{1, T_{1} T_{2}, \cdots, T_{1} T_{m}\right\}, \mathfrak{U}=\left\langle T_{1} T_{2}, \cdots, T_{1} T_{m}\right\rangle$. Then $\mathfrak{U}$ is a subgroup of $\mathfrak{M}$ normalized by $T_{1}$ and $\subseteq$ is a subset of $\mathfrak{M}$ closed under all power maps $X \mapsto X^{s}, s=1, \cdots$. Also, $|\mathfrak{u}|$ is odd since $T_{1}$ commutes with no involution of $\mathfrak{M}$. Let $p$ be a prime such that some element $S$ of $\subseteq$ has order $p$. If $S$ is a product of involutions $M, M^{\prime} \in \mathfrak{M}$, then since $M, M^{\prime} \in \boldsymbol{O}_{2^{\prime}, 2}(\mathfrak{M})$, we get $S \in \boldsymbol{O}_{2^{\prime}}(\mathfrak{M})$. But then Hypothesis 10.1(v) is violated. Hence, $S$ is not a product of two involutions of $\mathfrak{M}$, so by Hypothesis 10.1(iv), $S_{p}$-subgroups of $\mathfrak{M}$ are cyclic.

Let $\pi$ be the set of primes $p$ such that some element of $\subseteq$ has order $p$. By the preceding argument, if $p \in \pi$ and $\mathfrak{U}_{p}$ is a $T_{1}$-invariant $S_{p}$-subgroup of $\mathfrak{u}$, then $\mathfrak{U}_{p}$ is cyclic and $T_{1}$ inverts every element of $\mathfrak{U}_{p}$. Hence, $\mathfrak{B}=\Pi_{p} \mathfrak{U}_{p}$ is the direct product of its subgroups $\mathfrak{U}_{p}$, $p \in \pi$. It is easy to see that $\mathfrak{B} \subseteq \mathfrak{S}$, even though $\subseteq$ is not yet known to be a subgroup. If $V$ is any element of $\mathfrak{S}$, then $V$ is a $\pi$-element, so $T_{1}$ centralizes $\boldsymbol{O}_{\pi^{\prime}}(\mathfrak{l})$. As $T_{1}$ inverts every element of $\mathfrak{B}$, it follows that $\mathfrak{B}$ also centralizes $\boldsymbol{O}_{\pi^{\prime}}(\mathfrak{U})$. Hence, $\mathfrak{B} \triangleleft \mathfrak{U}$. Hence, $T_{1}$ centralizes $\mathfrak{U} / \mathfrak{B}$. Since $|\mathfrak{u}|$ is odd, and $\mathfrak{U}$ is generated by elements inverted by $T_{1}$, we have $\mathfrak{U}=\mathfrak{B}$. In particular, $|\mathfrak{B}|=m$. Since a $S_{\pi}$-subgroup of $\mathfrak{M}$ is a $Z$-group, and since no element of $\mathfrak{B}^{\sharp}$ centralizes any involution of $\mathfrak{M}$, it follows that $\mathfrak{M}=\mathfrak{C} \mathfrak{B}, \mathfrak{C} \cap \mathfrak{B}=1$, and this implies that $\mathfrak{C}$ is a $\pi^{\prime}$-group.

Let $\mathfrak{I}$ be a $S_{2}$-subgroup of $\mathfrak{M}$ permutable with $\mathfrak{B}$. Since $\mathfrak{I}$ has more than 1 involution, $\boldsymbol{O}_{2^{\prime}}(\mathfrak{I N})=1$. By Lemma 5.40, we conclude 
that $\mathfrak{T} \mathfrak{B}$ is a Frobenius group with Frobenius kernel $\mathfrak{T}$.

If $m_{0}$ is the number of involutions of $\mathfrak{T}$, then $m_{0} \leqq m$, clearly. On the other hand, $m_{0} \equiv 0(\bmod |\mathfrak{B}|)$, since $\mathfrak{T} \mathfrak{F}$ is a Frobenius group. Since $m=|\mathfrak{B}|$, it follows that $m=m_{0}$, so $\mathfrak{B}$ permutes transitively and regularly the involutions of $\mathfrak{M}$. Hence, $m=2^{k}-1$, where $2^{k}=$ $\left|\Omega_{1}(\boldsymbol{Z}(\mathfrak{T}))\right|$. Let $\mathfrak{S}_{0}=\Omega_{1}(\boldsymbol{Z}(\mathfrak{T}))$, so that $\mathfrak{S}_{0} \triangleleft \mathfrak{M}$, and let $\mathfrak{S}_{0}=\boldsymbol{C}_{\mathfrak{E}}\left(\mathfrak{S}_{0}\right)=$ $\boldsymbol{C}_{\mathfrak{m}}\left(\mathfrak{S}_{0}\right)$. Clearly, $\mathfrak{C}_{0}$ is a T.I. set in $\mathbb{S}$.

Since $\mathfrak{B}$ is transitive on $\mathfrak{S}_{0}^{*}$, any $\pi^{\prime}$-element of $\mathfrak{M}$ which centralizes $\mathfrak{B}$ lies in $\mathfrak{E}_{0}$. Let $\overline{\mathfrak{M}}=\mathfrak{M} / \mathfrak{S}_{0}$ and let $\overline{\mathfrak{B}}=\mathfrak{B} \mathfrak{E}_{0} / \mathfrak{E}_{0}$. As $\mathfrak{B}$ is a $S$-subgroup of $\mathfrak{M}$ and is transitive on $\mathfrak{S}_{0}^{*}$, it follows that $\boldsymbol{O}_{\pi^{\prime}}(\overline{\mathfrak{M}})=1$, which in turn implies that $\overline{\mathfrak{B}}=\boldsymbol{F}(\overline{\mathfrak{M}})$. Hence, the subgroups $C_{0}^{-1} \mathfrak{B} C_{0}, C_{0} \in \mathfrak{V}_{0}$, exhaust all conjugates of $\mathfrak{B}$ contained in $\mathfrak{M}$. Also, if $A, A_{1} \in \mathfrak{M}$, then $\left[\mathfrak{B}^{A}, \mathfrak{B}^{A_{1}}\right] \subseteq \mathfrak{S}_{0}$.

We next show that if $V \in \mathfrak{B}^{\sharp}$ and $G \in \mathbb{S}-\mathfrak{M}$, then $V^{G} \neq V$. As we have already shown, we have $G=C U, C \in \mathbb{E}, U$ an involution. Furthermore, there is an element $C_{0}$ of $\mathfrak{F}_{0}$ such that $U$ inverts every element of $C_{0}^{-1} \mathfrak{B} C_{0}$. Hence,

$$
\begin{gathered}
U C^{-1} V C U=V, \\
U C_{0}^{-1} V C_{0} U=C_{0}^{-1} V^{-1} C_{0} .
\end{gathered}
$$

Thus, $U\left[C^{-1} V C, C_{0}^{-1} V C_{0}\right] U=\left[V, C_{0}^{-1} V^{-1} C_{0}\right]$. Since $\mathbb{S}_{0}$ is a T.I. set in (8) and $\mathfrak{M}=N_{\leftrightarrow}\left(\mathfrak{S}_{0}\right)$, we have $\left[V, C_{0}^{-1} V^{-1} C_{0}\right]=1$. Hence, we necessarily have $C_{0}^{-1} V C_{0}=V$, since $\langle V\rangle$ is a $S$-subgroup of $\left\langle V, C_{0}^{-1} V C_{0}\right\rangle$. Hence (10.1) and (10.2) become $C^{-1} V C=U V U=V^{-1}$. This is not the case, since $\mathfrak{B}$ normalizes $\mathfrak{I}$, so that no element of $\mathfrak{B}^{\sharp}$ is $\mathfrak{M}$-real. Hence $\boldsymbol{C}_{\mathfrak{\Theta}}(V) \subseteq \mathfrak{M}$ for all $V$ in $\mathfrak{B}^{\sharp}$.

We next show that $C \mathfrak{C}_{0}(V)=1$ for all $V$ in $\mathfrak{B}^{\sharp}$. Since $C_{\mathfrak{G}}(V)=$ $\boldsymbol{C}_{\mathfrak{n}}(V)$, we see that $T_{1}$ normalizes $\boldsymbol{C}_{\mathfrak{n}}(V)$, so normalizes $\boldsymbol{O}_{\pi^{\prime}}\left(\boldsymbol{C}_{\mathfrak{n}}(V)\right) \supseteqq$ $C_{\mathfrak{n}}(V) \cap \mathfrak{S}_{0}$. Since $T_{1}$ normalizes $\mathfrak{B}, T_{1}$ also normalizes

$$
\left[\mathfrak{B}, \boldsymbol{O}_{\pi^{\prime}}\left(\boldsymbol{C}_{\mathfrak{n}}(V)\right)\right] \subseteq \mathfrak{\Im}_{0} .
$$

Since $\mathfrak{F}_{0}$ is a T.I. set in $\mathbb{B}$, we have $\left[\mathfrak{B}, \boldsymbol{O}_{\pi^{\prime}}\left(\boldsymbol{C}_{\mathfrak{m}}(V)\right)\right]=1$. Hence, $\mathfrak{B} \triangleleft C_{\mathfrak{n}}(V)$. Since $\mathfrak{B}$ is the largest subset of $\mathfrak{M}$ which is inverted by $T_{1}$, it follows that $T_{1}$ centralizes some $S_{\pi^{\prime}}$-subgroup of $C_{\mathfrak{m}}(V)$, and in particular, centralizes $C_{\oplus_{0}}(V)$. Hence $C_{\oplus_{0}}(V)=1$, and so $\mathfrak{C}_{0} \mathfrak{B}$ is a Frobenius group with Frobenius kernel $\mathfrak{C}_{0}$. Since $\mathfrak{I} \subseteq \mathfrak{C}_{0}$, $\mathfrak{I}$ is a T.I. set in 85 . We may apply [36] and conclude the proof of the theorem, since if $q=2^{n}>2, U_{3}(q)$ is not an $N$-group.

HYPOTHESIS 10.2. (a) $p, q \in \pi(\$)-\{2\}$.

(b) $\mathfrak{B}$ is a $S_{p}$-subgroup of $\mathbb{B S}$ and $\mathfrak{\Omega}_{0}$ is a maximal element of И(ホ⿱ $; q)$.

(c) $\mathfrak{P}$ does not centralize $\mathfrak{\Omega}_{0}$. 
(d) $\mathfrak{\beta} \subseteq N_{\circledast}\left(\Omega_{0}\right)^{\prime}$.

(e) $\mathfrak{\Omega}_{0}$ contains an element $Q$ of order $q$ such that $C_{\Theta}(Q) \cap N_{\Theta}\left(\Omega_{0}\right)$ does not contain an elementary subgroup of order $q^{3}$.

(f) $\mathfrak{Q}$ is a $S_{q}$-subgroup of $N_{\Theta}\left(\mathfrak{\Omega}_{0}\right)$ permutable with $\mathfrak{P}$.

Lemma 10.6. Under Hypothesis 10.2,

(i) $q \in \pi_{2}$.

(ii) $\mathfrak{Q}$ is a $S_{q}$-subgroup of (S).

(iii) Either $\mathfrak{Q}$ is abelian or $\mathfrak{Q}$ is a central product of a cyclic group and a nonabelian group of order $q^{3}$ and exponent $q$.

(iv) $\Omega_{1}(\mathfrak{Q}) \subseteq \mathfrak{\Omega}_{0}$.

Proof. Hypothesis $10.2(\mathrm{c})$ and (d) imply that $\boldsymbol{A}_{\mathscr{G}}\left(\mathfrak{\Omega}_{0}\right)$ is nonabelian. In particular, $\mathfrak{\Omega}_{0}$ is noncyclic. We first treat the case $m\left(\boldsymbol{Z}\left(\Omega_{0}\right)\right) \geqq 2$. By Hypothesis $10.2(\mathrm{e})$, it follows that $\Omega_{1}\left(Z\left(\Omega_{0}\right)\right)=\Omega_{1}\left(\Omega_{0}\right)$ is of type $(q, q)$. Let $\mathfrak{\Omega}^{*}$ be a $S_{q}$-subgroup of $\&$ which contains $\Omega$. Clearly,

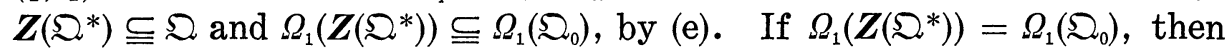
$\mathfrak{Q}=\mathfrak{\Omega}^{*}$, since $\mathfrak{Q}$ is $S_{q}$-subgroup of the normalizer of every nonidentity characteristic subgroup of $\Omega_{0}$. In this case, we have $\Omega_{1}\left(\Omega_{0}\right)=\Omega_{1}(\mathfrak{\Omega})$. In particular, $\mathscr{S C C}_{\mathfrak{3}}(\mathfrak{Q})$ is empty. Since $q>3$, an appeal to 0.3.4 shows that $\mathfrak{Q}$ is metacyclic, so by $0.3 .8, \mathfrak{Q}$ is abelian and we are done.

Suppose $\Omega_{1}\left(\boldsymbol{Z}\left(\mathfrak{\Omega}^{*}\right)\right) \subset \Omega_{1}\left(\mathfrak{\Omega}_{0}\right)$. In this case, we conclude that

$$
\Omega_{1}\left(Z_{2}\left(\Omega^{*}\right)\right) \subseteq \Omega
$$

If $\Omega_{1}\left(\boldsymbol{Z}_{2}\left(\Omega^{*}\right)\right)$ does not centralize $\Omega_{1}\left(\Omega_{0}\right)$, then the image of $\Omega_{1}\left(\boldsymbol{Z}_{2}\left(\Omega^{*}\right)\right)$ in $\boldsymbol{A}_{\oplus}\left(\Omega_{1}\left(\Omega_{0}\right)\right)$ is a normal subgroup of order $q$, so that $S_{q^{\prime}}$-subgroups of $\boldsymbol{A}_{\mathscr{\Theta}}\left(\Omega_{0}\right)$ are abelian and (d) is violated. Thus $\Omega_{1}\left(\boldsymbol{Z}_{2}\left(\Omega^{*}\right)\right)$ centralizes $\Omega_{1}\left(\Omega_{0}\right)$. Hence, $\Omega_{1}\left(Z_{2}\left(\Omega^{*}\right)\right)=\Omega_{1}\left(\Omega_{0}\right)$ by $(\mathrm{e})$. This in turn implies that $\mathfrak{\Omega}^{*}=\mathfrak{Q}$ and that $\mathscr{S C C}_{\mathfrak{S}}(\mathfrak{Q})$ is empty. Since $\mathfrak{Q}$ is nonabelian, $\mathfrak{Q}$ is not metacyclic. Hence, $\Omega_{1}(\Omega)$ is of order $q^{3}$, by 0.3 .4 . Since $\Omega_{1}\left(\Omega_{0}\right)$ is of index $q$ in $\Omega_{1}(\Omega)$, it follows that $\boldsymbol{A}_{\oplus}\left(\Omega_{0}\right)$ has a normal subgroup of order $q$ and (d) is violated. Thus, if $\boldsymbol{Z}\left(\mathfrak{\Omega}_{0}\right)$ is noncyclic, we are done.

The rest of the proof assumes that $Z\left(\mathfrak{\Omega}_{0}\right)$ is cyclic. By (d), $\mathfrak{\beta}$ centralizes $\boldsymbol{Z}\left(\mathfrak{\Omega}_{0}\right)$. Let $\Omega_{1}$ be an element of $\mathscr{B}\left(\Omega_{0}\right)$, and let $\Omega_{2}=\Omega_{1}\left(\Omega_{1}\right)$. Suppose $Q \notin \mathfrak{\Omega}_{2}$. Then $\boldsymbol{C}_{\mathfrak{Q}_{2}}(Q)$ is of order $q$, and $\left\langle Q, \mathfrak{\Omega}_{2}\right\rangle$ is of maximal class. This implies that $\gamma^{i} \mathfrak{\Omega}_{2} \mathfrak{N}_{0}^{i}$ is of index $q^{i}$ in $\mathfrak{\Omega}_{2}, i=1, \cdots, n$, $q^{n}=\left|\mathfrak{\Omega}_{2}\right|$. Now (d) implies that $\mathfrak{P}$ centralizes $\mathfrak{Q}_{2}$, so centralizes $\mathfrak{\Omega}_{1}$, so centralizes $\mathfrak{\Omega}_{0}$. This is not the case, so $Q \in \mathfrak{\Omega}_{2}$. As $\mathfrak{\Omega}_{2}$ is of class at most 2 and is of exponent $q$, this implies that $\left|\mathfrak{N}_{2}\right| \leqq q^{3}$. Suppose $\left|\mathfrak{\Omega}_{2}\right|=q^{2}$. We see that $\mathfrak{P}$ centralizes $\mathfrak{\Omega}_{2}$ and so centralizes $\mathfrak{\Omega}_{0}$. Hence, $\mathfrak{Q}_{2}$ is the nonabelian group of order $q^{3}$ and exponent $q$. Now (d) 
implies that $\boldsymbol{A}_{\mathfrak{\leftrightarrow}}\left(\mathfrak{\Omega}_{2} / \mathfrak{\Omega}_{2}^{\prime}\right)$ is a $q^{\prime}$-group. Hence, by a well-known property of extra special groups, we have $\mathfrak{Q}=\mathfrak{\Omega}_{2} C_{\mathfrak{Q}}\left(\mathfrak{\Omega}_{2}\right)$. By (e), $C_{\mathfrak{Q}}\left(\mathfrak{\Omega}_{2}\right)$ is cyclic. The proof is complete.

Hypothesis 10.3. (a) $p \in \pi_{3}-\{2\}$ and $\mathfrak{P}$ is a $S_{p}$-subgroup of $\mathbb{B}$.

(b) $q$ is an odd prime, $q \neq p$ and $\mathfrak{\Omega}_{0}$ is a maximal element of И(ঙ; $q)$.

(c) $\mathfrak{P}$ does not centralize $\mathfrak{\Omega}_{0}$.

(d) $\mathfrak{Q}$ is a $S_{q}$-subgroup of $N_{\mathscr{\Theta}}\left(\mathfrak{Q}_{0}\right)$ permutable with $\mathfrak{B}$.

(e) Either $q \neq 3$ or $3 \in \pi_{3}$.

Lemma 10.7. Under Hypothesis 10.3, $\mathfrak{D}$ is a $S_{q}$-subgroup of (3).

Proof. By Theorem 10.1, $\mathfrak{B} \subseteq N_{\circledast}\left(\mathfrak{\Omega}_{0}\right)^{\prime}$.

We may assume that Hypothesis 10.2 is not satisfied for $\left(\mathfrak{P}, \mathfrak{\Omega}_{0}, \mathfrak{\Omega}\right)$. Hence, $q \in \pi_{3} \cup \pi_{4}$. If $q \in \pi_{4}$, then since $q \geqq 5$, the lemma follows from Theorem 10.2. Suppose $q \in \pi_{3}$. In this case, the lemma follows from $\S 0.20$.

LEMmA 10.8. If $p, q \in \pi_{3}-\{2\}$ and $\langle\mathfrak{A}, \mathfrak{B}\rangle \in \mathscr{S O} \mathscr{L}(\mathbb{S})$ where $\mathfrak{A} \in$ $\mathscr{A}(p)$ and $\mathfrak{B} \in \mathscr{A}(q)$, then $p \sim q$.

Proof. Assume that $p \propto q$. Choose the notation so that $p>q$. The proofs of Lemma 0.10 .12 and 0.22 .1 show that if $\Omega$ is a $p, q$-subgroup of (S) and $\Omega$ contains an element of $\mathscr{A}(p)$, then $\Omega$ is $p$-closed. On the other hand, by sections 0.17 and 0.19 , it follows that if $\Re$ contains an elementary subgroup of order $q^{3}$, then $\Omega$ is $q$-closed. The details are straightforward, so are omitted.

We may assume that $\langle\mathfrak{A}, \mathfrak{B}\rangle$ is a $p, q$-group. Let $\Omega$ be a maximal $p, q$-subgroup of $\mathbb{S}$ containing $\langle\mathfrak{R}, \mathfrak{B}\rangle$, and let $\Re_{p} \Re_{q}$ be a Sylow system for $\Re$. We may assume that $\mathfrak{B} \subseteq \mathfrak{R}_{q}$. Clearly $\mathfrak{B}$ is faithfully represented on $\Omega_{p}$, since $p \nsim q$. We may therefore choose $B$ in $\mathfrak{B}^{\sharp}$ so that $C_{\mathfrak{A}_{p}}(B)$ is not centralized by $\mathfrak{B}$. Let $\mathbb{C}=\boldsymbol{C}_{\mathbb{\Theta}}(B)$ so that $\sqrt{5}$ contains an elementary subgroup of order $q^{3}$. Thus, all $p$, $q$-subgroups of $\sqrt{5}$ are $q$-closed. This violates the fact that $\mathfrak{B} C_{\mathfrak{R}_{p}}(B)$ is not $q$-closed and completes the proof.

Lemma 10.9. Assume the following:

(a) $p, q \in \pi_{3} \cup \pi_{4}-\{2\}$.

(b) Either $3 \notin\{p, q\}$ or $3 \in \pi_{3}$.

(c) $\langle\mathfrak{A}, \mathfrak{B}\rangle \in \mathscr{S O L} \mathscr{L}(\mathfrak{S})$, where $\mathfrak{U} \in \mathscr{A}(p), \mathfrak{B} \in \mathscr{A}(q)$.

Then $p \sim q$.

Proof. If $p$ and $q$ are in $\pi_{4}$, then $M(\langle\mathfrak{R}, \mathfrak{B}\rangle)$ contains a $S_{p, q^{-}}$ 
subgroup of (S), by Theorem 10.2. This is obviously impossible. We may assume that $p \in \pi_{4}, q \in \pi_{3}$, by Lemma 10.8. Let $\mathfrak{M}=\boldsymbol{M}(\langle\mathfrak{A}, \mathfrak{B}\rangle)$. Then $\mathfrak{M}$ contains a $S_{p}$-subgroup $\mathfrak{P}$ of $\mathbb{S}$. Let $\mathbb{L}$ be a $S_{p, q}$-subgroup of $\mathfrak{M}$ which contains $\mathfrak{P}$, and let $\mathfrak{Q}$ be a $S_{q}$-subgroup of $\mathfrak{Q}$. We may assume that $\mathfrak{B} \subseteq \Omega$.

Let $\mathfrak{P}_{0}=\boldsymbol{O}_{p}(\mathfrak{Q})$. Since $\boldsymbol{O}_{p^{\prime}}(\mathfrak{M})=1$, so also $\boldsymbol{O}_{p^{\prime}}(\mathfrak{Q})=1$, so $\mathfrak{Q}$ acts faithfully on $\mathfrak{P}_{0}$.

Suppose by way of contradiction that $\Omega$ contains no elementary subgroup of order $q^{3}$.

Choose $B \in \mathfrak{B}^{\sharp}$. We argue that one of the following holds:

$(\alpha) \quad C_{\mathfrak{\beta}_{0}}(B)$ is cyclic.

( $\beta) \quad C_{\mathfrak{\Re}_{0}}(B) \in \mathscr{A}_{4}(p)$.

Suppose $(\alpha)$ does not hold. Let $\mathfrak{E}=C_{\mathfrak{F}_{0}}(B), \mathfrak{R}=\boldsymbol{N}_{\mathfrak{\Re}_{0}}(\mathfrak{S})$. Since $\mathfrak{B}$ acts faithfully on $\mathfrak{P}_{0}$, we have $\mathfrak{R} \supset \mathfrak{E}$. Also, $\mathfrak{N}=\mathfrak{C}[\mathfrak{N},\langle B\rangle]$, and so $\mathfrak{R}=\left(\mathfrak{5} \cdot C_{\mathfrak{R}}(\mathfrak{S})\right.$. Since $B$ does not centralize $\mathfrak{R}, B$ does not centralize $\boldsymbol{C}_{\mathfrak{r}}(\mathbb{E})$, and so does not centralize $\Omega_{1}\left(\boldsymbol{C}_{\mathfrak{n}}(\mathbb{E})\right)$. Thus, $\boldsymbol{C}_{\mathfrak{r}}(\mathfrak{E})$ contains an element $D$ of order $p$ which is not in $\mathfrak{E}$, and so $\langle\mathbb{E}, D\rangle=\mathbb{E} \times\langle D\rangle$. This equality shows that $\left(\mathfrak{S} \in \mathscr{A}_{4}(p)\right.$.

Suppose now that $(\beta)$ holds for some $B$ in $\mathfrak{B}^{\sharp}$. Then $C(B) \cap \mathfrak{M}$ contains an element of $\mathscr{A}_{4}(p)$, and so $C(B) \subseteq \mathfrak{M}$. But $\mathfrak{B} \in \mathscr{A}_{4}(q)$, and so $C(B)$ contains an elementary subgroup of order $q^{3}$, the desired contradiction.

Since $\mathfrak{B}$ acts faithfully on $\mathfrak{P}_{0}$, we can choose $B \in \mathfrak{B}^{\sharp}$ such that $\mathfrak{B}$ does not centralize $C_{\mathfrak{\beta}_{0}}(B)$. Since $\boldsymbol{C}_{\mathfrak{\beta}_{0}}(B)$ is cyclic, we get $p \equiv 1$ $(\bmod q)$. In particular, $\mathfrak{P}_{0} \mathfrak{B}$ is supersolvable.

Let $\mathfrak{D}$ be a minimal normal subgroup of $\mathfrak{P}_{0} \mathfrak{B}$. Thus, $\mathfrak{D} \subseteq Z\left(\mathfrak{P}_{0}\right)$, and $|\mathfrak{D}|=p$. Also, $\mathfrak{P}_{0}=C_{\mathfrak{P}_{0}}(\mathfrak{D})$ contains an elementary subgroup of order $p^{3}$, so $\boldsymbol{C}(\mathfrak{D}) \subseteq \mathfrak{M}$.

Let $\mathfrak{B}_{0}=\mathfrak{B} \cap \boldsymbol{C}(\mathfrak{D})$, so that $\left|\mathfrak{B}_{0}\right|=q$. Let $\Re$ be a $S_{p, q}$-subgroup of $\boldsymbol{C}\left(\mathfrak{B}_{0}\right)$ which contains $\mathfrak{B D}$. Then

(i) $\Omega$ is not $q$-closed.

(ii) $\Omega$ contains an elementary subgroup of order $q^{3}$. Let $\mathfrak{S}$ be a $p, q$-subgroup of $\mathbb{B S}$ which satisfies (i) and (ii) and such that $\left|\mathfrak{S}_{\mathcal{C}}\right|_{q}$ is maximal. Let $\mathfrak{S}_{p}, \mathfrak{S}_{q}$ be a Sylow system for $\mathfrak{F}_{\mathcal{C}}$.

Case 1.

$$
\left[O_{p}(\mathfrak{K}), \mathscr{S}_{q}\right]=1 \text {. }
$$

In this case, $\boldsymbol{O}_{p, q}(\mathfrak{S})=\boldsymbol{O}_{p}(\mathfrak{S}) \times \boldsymbol{O}_{q}(\mathfrak{S})$. By Theorem 1 of [43], we get that $\mathscr{K}_{q}$ is a $S_{q}$-subgroup of $\mathbb{S}$. Set $\mathscr{S}_{0}=\boldsymbol{O}_{q}(\mathfrak{C})$.

Since $p \nsim q, S_{p}$-subgroups of $\mathfrak{S}_{\mathcal{C}}$ are not in $\mathscr{A}_{4}(p)$. Thus, $\mathfrak{F}_{p}$ has a cyclic subgroup $\mathfrak{F}^{5}$ of index $p$. Since $q \in \pi_{3}, \mathscr{S}_{q} \subseteq N\left(\mathscr{S}_{0}\right)^{\prime}$.

Now $\mathfrak{S}_{p}$ has a cyclic subgroup of index $p$ and $\mathscr{S}$ is a $S_{p, q}$-subgroup of $N\left(\mathfrak{S}_{0}\right)$, so $l_{p}\left(N\left(\mathfrak{S}_{0}\right)\right)=1$. Since $\mathfrak{S}_{q} \subseteq N\left(\mathfrak{F}_{0}\right)^{\prime}$, it follows that $\mathscr{A}_{N\left(\mathfrak{F}_{0}\right)}\left(\mathfrak{S}_{p}\right)$ is of order divisible by $q$, and that $\mathscr{A}_{N\left(\mathfrak{F}_{0}\right)}\left(\mathfrak{S}_{p}\right)^{\prime}$ contains 
a $S_{q}$-subgroup of $\mathscr{A}_{N\left(\mathfrak{F}_{0}\right)}\left(\mathfrak{S}_{p}\right)$. This implies that $\mathfrak{S}_{p}$ is elementary of order $p^{2}$.

Let $\mathfrak{X}=\boldsymbol{A}_{\Theta}\left(\mathfrak{S}_{p}\right)$. Since $\mathfrak{S}_{p} \notin \mathscr{A}_{4}(p)$, it follows that $p|| \mathfrak{X} \mid$. Since $\mathfrak{X}$ is solvable, $\mathfrak{X}^{\prime}$ is a $p$-group. This is the desired contradiction, since $q\left|\mathfrak{X}^{\prime}\right|$.

$$
\text { Case 2. } \quad\left[\boldsymbol{O}_{p}\left(\mathfrak{S}_{\mathcal{C}}\right), \mathfrak{S}_{q}\right] \neq 1 .
$$

Since $\mathscr{S}$ contains no element of $\mathscr{A}(p)$, we get $\left|\boldsymbol{O}_{p}(\mathfrak{F})\right|=p$. Hence, $\left|\boldsymbol{O}_{q}(\mathfrak{S})\right|>q$, since $\mathfrak{H}_{q}$ contains an elementary subgroup of order $q^{3}$. By Lemma 6.6 (ii) (with $p$ and $q$ interchanged), it follows that $\mathfrak{K}_{q}$ is a $S_{q}$-subgroup of $\left(\mathcal{S}\right.$, and $\boldsymbol{O}_{p}\left(\mathfrak{S}_{\mathcal{L}}\right)$ is a maximal element of $N\left(\mathfrak{S}_{q} ; p\right)$. By Theorem 10.1, we have $\mathscr{S}_{q} \subseteq N\left(O_{p}(\mathfrak{K})\right)^{\prime}$, which is the desired contradiction. The proof is complete.

Lemma 10.10. Assume the following:

(a) $p, q \in \pi_{3} \cup \pi_{4}-\{2\}$.

(b) Either $p \neq 3$ or $3 \in \pi_{3}$.

(c) There is an element $\mathfrak{S}_{2}$ in $\mathscr{S O S} \mathscr{L}(\mathbb{S})$ such that

(i) $\mathfrak{S}$ contains an element of $\mathscr{A}(p)$.

(ii) $\mathfrak{S}$ is a p, q-group and $\mathfrak{S}$ is not p-closed.

Then $p \sim q$.

The proof of Lemma 10.10 parallels the proof of Lemma 10.9 and is therefore omitted.

We are now in a position to handle adequately.

LEMMA 10.11. Assume the following:

(a) $p, q, r \in \pi_{3} \cup \pi_{4}-\{2\}$.

(b) Either $3 \neq p, q, r$ or $3 \in \pi_{3}$.

(c) $p \sim q$ and $p \sim r$.

Then $q \sim r$.

Proof. We may assume that $\langle\mathfrak{A}, \mathfrak{B}\rangle$ is nonsolvable whenever $\mathfrak{U} \in \mathscr{A}(q)$ and $\mathfrak{B} \in \mathscr{A}(r)$. Let $\mathfrak{P}$ be a $S_{p}$-subgroup of $\mathbb{S}$. By sections 0.19 and 0.20 together with Theorem 10.1, (S) satisfies $E_{p, q}$ and $E_{p, r}$. Let $\mathfrak{\Omega}, \Re$ be $S_{q}$-and $S_{r}$-subgroups of (s) respectively which are permutable with $\mathfrak{B}$. Suppose $\mathfrak{B}$ centralizes $\boldsymbol{O}_{q}(\mathfrak{P} \mathfrak{Q})$, and $\boldsymbol{O}_{q}(\mathfrak{P} \Omega)$ is cyclic. If $\boldsymbol{O}_{r}(\mathfrak{P} \Re) \neq 1$, then by the transitivity theorem, $\boldsymbol{N}_{\circledast}\left(\boldsymbol{O}_{r}(\mathfrak{B} \Re)\right)$ contains an element of $\mathscr{A}(q)$. If $\boldsymbol{O}_{r}(\mathfrak{B R})=1$, then $\boldsymbol{N}_{\mathfrak{\Theta}}(\mathfrak{B})$ contains elements of $\mathscr{A}(q)$ and $\mathscr{A}(r)$ where $\mathfrak{B}=V\left(\operatorname{ccl}_{\mathscr{N}}(\mathfrak{l}) ; \mathfrak{P}\right), \mathfrak{A} \in \mathscr{S} \mathscr{C} \mathscr{N}_{3}(\mathfrak{P})$. Suppose $\mathfrak{P}$ centralizes $\boldsymbol{O}_{q}(\mathfrak{P} \mathfrak{Q})$ and $\boldsymbol{O}_{q}(\mathfrak{P} \mathfrak{Q})$ is noncyclic. Then $\boldsymbol{O}_{r}(\mathfrak{P} \Re)$ is cyclic, since otherwise $C_{\mathscr{G}}(P)$ contains an element of $\mathscr{A}(q)$ and of $\mathscr{A}(r)$ for some $P$ in $\mathfrak{S}^{\sharp}$. Since $\boldsymbol{O}_{r}(\mathfrak{P} \Re)$ is cyclic, it follows that $\boldsymbol{N}_{\Theta}\left(\boldsymbol{O}_{q}(\mathfrak{P} \mathfrak{Q})\right)$ 
contains an element of $\mathscr{A}(r)$. Thus, we may assume that $\mathfrak{P}$ does not centralize $\boldsymbol{O}_{q}(\mathfrak{B} \mathfrak{Q})$ and $\mathfrak{P}$ does not centralize $\boldsymbol{O}_{r}(\mathfrak{P} \Re)$. By Lemma 10.6 if $Q$ is an element of $\boldsymbol{O}_{q}(\mathfrak{B} \Omega)$ of order $q$, then $\boldsymbol{C}_{\mathbb{Q}}(Q)$ contains an elementary subgroup of order $q^{3}$. Similarly for $\boldsymbol{O}_{r}(\mathfrak{P} \Re)$. We may now copy the proof of Theorem 0.22.2. The proof is complete.

Lemma 10.12. (i) Suppose $p, q \in \pi_{3}-\{2\}$ and \&s satisfies $E_{p, q}$. Then (S) satisfies $C_{p, q}^{k}$.

(ii) Suppose $p, q \in \pi_{2}-\{2\}$ and \&s satisfies $E_{p, q}$. Then (S) satisfies $D_{p, q}$.

Proof. The proof of (i) may be left to the reader, as it parallels the proofs in $\S 0.21$. As for (ii), if $p>q$, then every $p, q$-subgroup of (S) is $p$-closed, so $D_{p, q}$ is obvious.

Definition 10.1. Let $\sigma$ be an equivalence class of $\pi_{3}-\{2\}$ under $\sim$ Then $\tau=\tau(\sigma)$ is the set of odd primes $q$ with the properties:

(a) $q \notin \sigma$

(b) $q \notin \pi_{4}$.

(c) There are at least one prime $p$ in $\sigma$ and one element $\subseteq$ of $\mathscr{S O S}\left(\mathbb{L}\right.$ (S) such that $\mathfrak{S}$ is $p, q$-group, $|\mathfrak{S}|_{p}=|\mathbb{S}|_{p}$, and $\mathbb{S}$ is not $p$ closed.

Lemma 10.13. Suppose $\sigma$ is an equivalence class of $\pi_{3}-\{2\}$ under $\sim$, and $p \in \sigma$. If $\mathfrak{S}_{0}$ is a $p, q$-subgroup of (B) which is not p-closed, if $\mathfrak{S}_{0}$ contains an element of $\mathscr{A}(p)$, and if $q \notin \sigma \cup \pi_{4} \cup\{2\}$, then $q \in$ $\tau(\sigma)$.

Proof. Let $\mathscr{S}$ be the set of subgroups of $(S)$ which satisfy the hypothesis of the lemma. Let $i$ be the smallest positive integer such that some element of $\mathscr{S}$ contain an element of $\mathscr{A}_{i}(p)$. Among all elements of $\mathscr{S}$ which contains an element of $\mathscr{A}_{i}(p)$, choose $\mathfrak{S}$ so that $|\mathfrak{S}|_{p}$ is maximal, and with this restriction, maximize $|\mathfrak{S}|_{q}$.

Suppose $i=1$. Choose $\mathfrak{U} \subseteq \mathfrak{S}$ such that $\mathfrak{U} \in \mathscr{S} \mathscr{C} \mathscr{N}_{3}(p)$, and $\mathfrak{S}_{p}$ is a $S_{p}$-subgroup of $\subseteq$ containing $\mathfrak{A}$ and let $\mathfrak{P}$ be a $S_{p}$-subgroup of $\mathbb{S}$ containing $\mathfrak{S}_{p}$. If $\mathfrak{A}$ centralizes $\boldsymbol{O}_{q}(\mathfrak{S})$, then $\mathfrak{S} \subseteq N_{\mathfrak{S}}(\mathfrak{B})$,

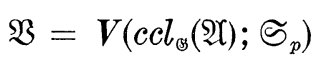

by section 0.17 , so by maximality of $\mathfrak{S}_{p}$, we have $\mathfrak{S}_{p}=\mathfrak{P}$ and we are done. If $\mathfrak{U}$ does not centralize $\boldsymbol{O}_{q}(\mathfrak{S})$, then maximality of $|\mathfrak{S}|_{q}$ guarantees that $\boldsymbol{O}_{q}(\mathfrak{S})$ is a maximal element of $\boldsymbol{U}(\mathfrak{H} ; q)$, so by maximality of $\mathfrak{S}_{p}$ and section $0.17, \Im_{p}=\mathfrak{P}$ and we are done.

Suppose $i>1$. Let $\mathfrak{\Omega}_{0}=\boldsymbol{O}_{q}(\mathfrak{S})$. If $\mathfrak{S}_{p}$ centralizes $\mathfrak{\Omega}_{0}$, then maxi- 
mality of $\mathfrak{S}_{p}$ and Lemma 0.7.9 guarantee that $\mathfrak{S}_{p}$ contains an element of $\mathscr{S C C N}_{3}(p)$, contrary to $i>1$. Hence, $\mathfrak{S}_{p}$ does not centralize $\mathfrak{\Omega}_{0}$. Let $\mathfrak{B}$ be a subgroup of $\mathfrak{S}_{p}$ of type $(p, p)$ with $\mathfrak{B} \in \mathscr{A}_{i}(\mathfrak{P})$. If $\mathfrak{B}$ does not centralize $\mathfrak{\Omega}_{0}$, then $\mathfrak{B}$ does not centralize $C_{\mathfrak{\Omega}_{0}}(B)$ for suitable $B$ in $\mathfrak{B}^{\sharp}$, so that $S_{p, q}$-subgroups of $C_{\leftrightarrow}(B)$ are not $p$-closed. This violates the choice of $i$, since $C_{\mathscr{\Theta}}(B)$ contains an element of $\mathscr{A}_{i-1}(p)$. Hence $\mathfrak{P}_{0}=\langle\mathfrak{B}| \mathfrak{B} \subseteq \mathfrak{S}_{p}, \mathfrak{B} \in \mathscr{A}_{i}(\mathfrak{P}), \mathfrak{B}$ of type $\left.(p, p)\right\rangle$ centralizes $\mathfrak{\Omega}_{0}$. Since $\mathfrak{B}_{0}$ is weakly closed in $\mathfrak{S}_{p}$, we have $\left.\left|\boldsymbol{N}_{\mathfrak{\leftrightarrow}}\left(\mathfrak{B}_{0}\right)\right|_{p}\right\rangle|\mathfrak{S}|_{p}$. Since $\mathfrak{S}_{p} \mathfrak{\Omega}_{0}$ is contained in $N_{\Theta}\left(\mathfrak{P}_{0}\right), S_{p q}$-subgroups of $N_{\Theta}\left(\mathfrak{P}_{0}\right)$ are not $p$-closed. This violates the choice of $\mathfrak{S}$ and completes the proof.

THEOREM 10.5. If $\sigma$ is an equivalence class of $\pi_{3}-\{2\}$ under $\sim$, then \&s satisfies $C_{\sigma \cup \tau(\sigma)}$ and $\tau(\sigma) \leqq \pi_{2}$.

Proof. Choose $q \in \tau(\sigma)$ and then choose $p \in \sigma$ so that for some $p, q$-subgroup $\subseteq$ of $\mathbb{S},|\mathfrak{S}|_{p}=|\mathbb{S}|_{p}$ and $\subseteq$ is not $p$-closed. We assume without loss of generality that $\mathfrak{S}$ is a maximal $p, q$-subgroup of $\mathbb{B S}$. Let $\left\{\mathfrak{S}_{p}, \mathfrak{S}_{q}\right\}$ be a Sylow system for $\mathfrak{S}$, and let $\mathfrak{\Omega}_{0}=\boldsymbol{O}_{q}(\mathfrak{S})$. If $\mathfrak{S}_{p}$ does not centralize $\mathfrak{\Omega}_{0}$, we apply Lemma 10.6 and conclude that $q \in$ $\pi_{2}$ and that $\mathfrak{S}_{q}$ is a $S_{q}$-subgroup of (S. In this case, it is straightforward to verify $C_{p, q}^{*}$. Suppose $\left[\mathfrak{S}_{p}, \mathfrak{\Omega}_{0}\right]=1$.

Let $\mathfrak{P}_{0}=\boldsymbol{O}_{p}(\mathfrak{S})$ so that $\boldsymbol{F}(\mathfrak{S})=\mathfrak{P}_{0} \times \mathfrak{\Omega}_{0}=\boldsymbol{O}_{q p}(\mathfrak{S})$. Let

$$
\mathfrak{U} \in \operatorname{SCCN}_{3}\left(\mathfrak{S}_{p}\right) \text {. }
$$

Thus, $\mathfrak{A} \subseteq \mathfrak{P}_{0}$, so $\subseteq \subseteq N_{\circledast}(\mathfrak{B})=\mathfrak{N}, \mathfrak{B}=V\left(\operatorname{ccl}_{\mathfrak{\Theta}}(\mathfrak{A}) ; \mathfrak{S}_{p}\right)$, and $\subseteq$ is a $S_{p, q^{-}}$ subgroup of $\mathfrak{N}$. By sections $0.17, \mathfrak{N}=\boldsymbol{O}^{p}(\mathfrak{N})$. Consider $\mathfrak{Q}=\boldsymbol{O}_{p q}(\mathfrak{S}) / \boldsymbol{O}_{p}(\mathfrak{S})$. Since $q \notin \sigma \cup \pi_{4}$, it follows that $\mathscr{S C C}_{\mathfrak{N}}(\mathfrak{\Omega})=\varnothing$. By construction, $\mathfrak{S}_{p} / \mathfrak{P}_{0}$ is faithfully represented on $\Omega$ and $\boldsymbol{A}_{\circledast}(\mathfrak{\Omega})=\boldsymbol{O}^{p}\left(\boldsymbol{A}_{\mathscr{\leftrightarrow}}(\mathfrak{\Omega})\right)$. This implies that $\boldsymbol{A}_{\mathfrak{\Theta}}(\mathfrak{\Omega} / \boldsymbol{D}(\mathfrak{\Omega}))$ is a $q^{\prime}$-group, which in turn implies that $\boldsymbol{O}_{p, q}(\mathfrak{S})=\mathfrak{B}_{0} \mathfrak{S}_{q}$, which in turn implies that $\mathfrak{S}_{q}$ is a $S_{q}$-subgroup of (S). The details are omitted.

Since $\Omega$ is clearly not cyclic, it follows that $q \in \pi_{2}$. Furthermore, it follows that $\&$ satisfies $C_{p, q}$, and the preceding argument shows that if $\mathfrak{I}$ is a maximal $p, q$-subgroup of $\mathbb{S}$ and $|\mathfrak{I}|_{p}=|\mathbb{S}|_{p}$, then $\mathfrak{I}$ is a $S_{p, q^{-}}$-subgroup of $\&$. (Note that this is weaker than $C_{p, q}^{*}$ ) In any case, we have shown that $\tau(\sigma) \subseteq \pi_{2}$.

Retaining the previous notation, let $p_{1} \in \sigma$. We may use Lemma 10.8 together with $C_{p, p_{1}}^{*}$ to conclude that $\&$ satisfies $C_{p_{1}, q}$.

Now suppose $q_{1} \in \tau(\sigma)$. By the preceding argument, (S) satisfies $C_{p, q_{1}}$. As the situation is symmetric in $q, q_{1}$, we may assume $q>q_{1}$. In this case, every $q, q_{1}$-subgroup of $\mathbb{B}$ is $q$-closed. Lemma $10.8, C_{p, q}$ and $C_{p, q_{1}}$ yield $C_{q, q_{1}}$.

We may now apply the argument of section 0.21 and conclude the proof. 
THEOREM 10.6. Suppose $p \in \pi_{3}, p$ is odd, $\mathfrak{P}$ is a $S_{p}$-subgroup of (S) and $\Re=\left\langle\mathcal{S} \mid \mathfrak{S} \in \mathcal{U}_{\mathfrak{S}}(\mathfrak{P})\right\rangle$ is a solvable $p^{\prime}$-group. Then

$$
\mathscr{A}(p) \subseteq \mathscr{C}^{*}(\mathfrak{S}) \text {. }
$$

Proof. Let $\mathfrak{M}=N_{\mathfrak{B}}(\mathfrak{R}) \supseteqq \mathfrak{P}$ and let $\mathscr{A}_{i}{ }^{*}(\mathfrak{P})=\{\mathfrak{A} \mid \mathfrak{A} \subseteq \mathfrak{P}$, $\mathfrak{A}$ contains an element of $\mathscr{A}_{i}\left(\mathfrak{P}^{M}\right)$ for some $M$ in $\left.\mathfrak{M}\right\}, i=1,2,3,4$, and let $i$ be the smallest integer such that some element of $\mathscr{A}_{i}^{*}(\mathfrak{P})$ is not in $\mathscr{C}^{*}(\mathbb{S})$. Let $\mathfrak{A}$ be such an element of maximal order and let $\mathfrak{S}$ be a solvable subgroup of $\mathbb{B S}$ which contains $\mathfrak{A}$, is not contained in $\mathfrak{M}$ and is minimal subject to these restrictions. Then $\subseteq=\mathfrak{A} \Omega$ for some $q$-group $\mathfrak{D}, q \neq p$. By minimality of $i$, together with the transitivity theorem, it follows that $\boldsymbol{O}_{q}(\mathfrak{S}) \subseteq \mathfrak{M}$. Hence, $\boldsymbol{O}_{q, p}(\mathfrak{S})=\boldsymbol{O}_{q}(\mathfrak{S}) \times \boldsymbol{O}_{p}(\mathfrak{S})$ and $\mathfrak{A}$ is a $S_{p}$-subgroup of $N_{\oplus}\left(O_{p}(S)\right)$. Let $\mathfrak{A}_{0}=\boldsymbol{O}_{p}(\mathfrak{S})$. Thus,

$$
\boldsymbol{C}_{\mathfrak{G}}\left(\mathfrak{U}_{0}\right)=\boldsymbol{Z}\left(\mathfrak{\Re}_{0}\right) \times \mathfrak{D},
$$

where $\mathfrak{D}$ is a $p^{\prime}$-group. Choose $S$ in $\mathfrak{S}-\mathfrak{M}$. Then $\mathfrak{A}_{0}$ normalizes $\Re^{S}$. Since $\mathfrak{R}^{S} \subseteq \mathfrak{M}$, it follows from Theorem 2 of [41] that

$$
\Re^{s} \subseteq \boldsymbol{O}_{p^{\prime}}(\mathfrak{M})=\Re \text {. }
$$

Hence $\Re=\AA^{S}$ against our choice of $S$. The proof is complete.

THEOREM 10.7. Suppose $\mathfrak{T}$ is a $S_{2}$-subgroup of $\mathbb{S}, 2 \in \pi_{3}$, and

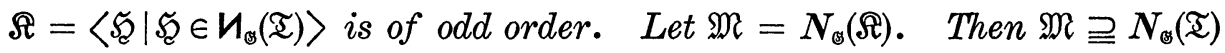
and $\mathfrak{M}$ contains the centralizer of each of its involutions.

Proof. If $N \in N_{\mathscr{\Theta}}(\mathfrak{T})$, then $\mathfrak{I}$ normalizes $\Re^{N}$. By definition of $\Re$, we have $\Re=\Re^{N}$, so $N_{\circledast}(\mathfrak{T}) \subseteq \mathfrak{M}$.

Suppose $Z \in \Omega_{1}(Z(\mathfrak{I}))^{\#}$. We will show that $\boldsymbol{C}_{\mathfrak{s}}(Z) \subseteq \mathfrak{M}$. Let

$$
\mathfrak{I}_{0}=\mathfrak{I} \cap \boldsymbol{O}_{2^{\prime}, 2}\left(C_{\Theta}(Z)\right) \text {. }
$$

Thus, there is a subgroup $\mathfrak{B}$ of $\mathfrak{I}_{0}$ with $Z \in \mathfrak{B} \in \mathscr{U}(\mathfrak{T})$. Choose $N \in$ $N\left(\mathfrak{I}_{0}\right) \cap C_{\Theta}(Z)$. Thus, $\mathfrak{I}_{0}$ normalizes $\AA^{N}$. Now $\Re^{N} \cap C_{\Theta}(Z)$ is normalized by $\mathfrak{T}_{0}$, so $\Re^{N} \cap C_{\circledast}(Z) \subseteq O_{2^{\prime}}\left(C_{\oplus}(Z)\right)$, so $\Re^{N} \cap C_{\Theta}(Z) \subseteq \Re$. Choose $B \in \mathfrak{B}^{\sharp}$. By Lemma 6.1, [ $\left.\Re^{N} \cap C(B), Z\right] \subseteq O_{2^{\prime}}\left(C_{\mathscr{G}}(B)\right)$. Since $C_{\mathscr{G}}(B)$ contains an element of $\mathscr{S C C N}_{3}(\mathfrak{T})$, it follows that $\left[\Re^{N} \cap C(B), Z\right] \subseteq \Re$. Hence $\Re^{N} \subseteq \Re$, so $\Re^{N}=\Re$, or in other words, $N \in \mathfrak{M}$. Since

$$
C_{\Theta}(Z)=O_{2^{\prime}}\left(C_{\circledast}(Z)\right) \cdot\left(N_{\Theta}\left(\mathfrak{I}_{0}\right) \cap C_{\Theta}(Z)\right),
$$

it follows that $C_{\oplus}(Z) \subseteq \mathfrak{M}$.

We next show that if $\mathfrak{A} \triangleleft \mathfrak{I}, C_{\mathfrak{E}}(\mathfrak{R})=Z(\mathfrak{U})$ and $\mathfrak{A}$ contains an element $\mathfrak{B}$ of $\mathscr{U}(\mathfrak{T})$, then $N_{\mathscr{\leftrightarrow}}(\mathfrak{Z}) \subseteq \mathfrak{M}$. Choose $N \in N_{\mathfrak{S}}(\mathfrak{R})$. Thus, $\mathfrak{A}$ normalizes $\mathfrak{R}^{N}$. Choose $Z \in Z(\mathfrak{T}) \cap \mathfrak{B}^{\sharp}$. Since $\left[\Re^{N} \cap C(B), Z\right] \subseteq \Re$ for 
all $B$ in $\mathfrak{B}^{\sharp}$ and since $\Re^{N} \cap \boldsymbol{C}(Z) \subseteq \Re$, it follows that $\Re^{N}=\Re, N \in \mathfrak{M}$ so $\boldsymbol{N}_{\mathfrak{G}}(\mathfrak{Q}) \subseteq \mathfrak{M}$.

Suppose $\mathfrak{I} \subseteq \subseteq \in \mathscr{S O} \mathscr{L}(\mathbb{S})$. Then $\boldsymbol{O}_{2^{\prime}}(\mathfrak{S}) \subseteq \Re$ and

$$
N\left(\mathfrak{I} \cap \boldsymbol{O}_{2^{\prime}, 2}(\subseteq)\right) \subseteq \mathfrak{M},
$$

so $\subseteq \subseteq \mathfrak{M}$. That is, $\mathfrak{I} \in \mathscr{M}^{*}(\mathbb{S})$.

Throughout the remainder of the proof, we set $\mathfrak{I}_{0}=\mathfrak{I} \cap \boldsymbol{O}_{2^{\prime}, 2}(\mathfrak{M})$. Since $2 \in \pi_{3}$, there is an element $\mathfrak{B}$ of $\mathscr{U}(\mathfrak{I})$ with $\mathfrak{B} \subseteq \mathfrak{I}_{0}$. We will show that $C_{\mathfrak{G}}(B) \subseteq \mathfrak{M}$ for all $B$ in $\mathfrak{B}^{\sharp}$. If $\boldsymbol{Z}(\mathfrak{T})$ is noncyclic, the assertion holds since $\mathfrak{I} \in \mathscr{C}^{*}(\mathbb{S})$. We may assume that $\boldsymbol{Z}(\mathfrak{T})$ is cyclic and that $B \notin \boldsymbol{Z}(\mathfrak{T})$. Let $\mathfrak{T}^{*}$ be a $S_{2}$-subgroup of $\boldsymbol{C}_{\mathfrak{m}}(B)$ which contains $C_{\varepsilon}(B)$. If $\mathfrak{I}^{*}$ is a $S_{2}$-subgroup of $\mathbb{S}$, then since $\mathfrak{I}^{*} \in \mathscr{M}^{*}(\mathfrak{S})$, we get $C_{\Theta}(B) \subseteq \mathfrak{M}$. We may assume that $\mathfrak{I}^{*}$ is of index 2 in a $S_{2}$-subgroup of $\mathfrak{M}$, so that $\mathfrak{I}^{*}=C_{\mathfrak{x}}(B)$. Since $N_{\mathbb{\Theta}}\left(\mathfrak{I}^{*}\right) \subseteq \mathfrak{M}$, it follows that $\mathfrak{I}^{*}$ is a $S_{2}$-subgroup of $C_{\circledast}(B)$.

Let $\mathfrak{I}_{1}=\mathfrak{I}^{*} \cap \boldsymbol{O}_{2^{\prime}, 2}(C(B))$ and choose $N$ in $C(B) \cap N\left(\mathfrak{I}_{1}\right)$. Then $\mathfrak{I}_{1}$ normalizes $\Re^{N}$. Let $\tilde{\Re}=\Re^{N} \cap C(B)$. By definition of $\mathfrak{T}_{1}$, it follows that $\tilde{\mathbb{R}} \subseteq \boldsymbol{O}_{2^{\prime}}(C(B))$. But $\mathfrak{I}^{*}$ contains an element of $\mathscr{S} \mathscr{C} \mathscr{N}_{3}(\mathfrak{I})$, so $\boldsymbol{O}_{2^{\prime}}(\boldsymbol{C}(B)) \subseteq \Re$. Thus, $\tilde{\Re} \subseteq \Re$. If $B_{1} \in \mathfrak{B}^{\sharp}$ and $Z \in \mathfrak{B}^{\sharp} \cap \boldsymbol{Z}(\mathfrak{I})$, then

$$
\left[\Re^{N} \cap C\left(B_{1}\right), Z\right] \subseteq \Re
$$

by Lemma 6.1. Since $\tilde{\mathscr{R}} \subseteq \Re$, it follows that $\mathfrak{R}^{N}=\mathfrak{R}$, so $C_{\mathscr{\Theta}}(B) \subseteq \mathfrak{M}$, as asserted.

Let $I$ be an involution of $\mathfrak{I}_{0}$ which centralizes a subgroup $\mathfrak{B}$ of $\mathscr{U}(\hat{\mathfrak{T}})$, where $\mathfrak{B} \subseteq \mathfrak{I}_{0}$ and $\hat{\mathfrak{I}}$ is some $S_{2}$-subgroup of $\mathfrak{M}$. Let $\mathfrak{I}_{1}$ be a $S_{2}$-subgroup of $C_{\mathfrak{n}}(I)$ which contains $C_{\mathbb{x}_{0}}(I)$. Let $\mathscr{F}=\{\tilde{\mathfrak{I}} \mid \tilde{\mathfrak{I}}$ is a 2subgroup of $\mathbb{S}$ and $\left.\widetilde{\mathfrak{I}} \supseteqq \mathfrak{I}_{1}\right\}$. We will show that each element of $\mathscr{F}$ is in $\mathfrak{M}$. Suppose false. Among all elements of $\mathscr{F}$ which are not contained in $\mathfrak{M}$, choose $\tilde{\mathfrak{I}}$ so that $\tilde{\mathfrak{I}} \cap \mathfrak{M}=\mathfrak{I}_{1}^{*}$ is maximal. Let $\mathfrak{I}^{*}$ be a $S_{2}$-subgroup of $N_{\mathfrak{M}}\left(\mathfrak{I}_{1}^{*}\right)$. Maximality of $\mathfrak{I}_{1}^{*}$ guarantees that $\mathfrak{I}^{*}$ is a $S_{2}$-subgroup of $N_{\Theta}\left(\mathfrak{T}_{1}^{*}\right)$. Choose $N$ in $N_{\circledast}\left(\mathfrak{T}_{1}^{*}\right)$. Then $\mathfrak{I}_{1}^{*}$ normalizes $\Re^{N}$, so $C_{\check{\Sigma}}(I)$ normalizes $\Re^{N}$. Since $\mathfrak{B} \subseteq C_{\mathscr{\Upsilon}_{0}}(I)$, it follows that $\Re^{N} \subseteq \mathfrak{M}$. By definition of $\mathfrak{I}_{0}$, it follows that $\left[\Re^{N}, C_{\check{x}_{0}}(I)\right] \subseteq \Re$. We will show that $\mathfrak{R}^{N} \cap \boldsymbol{C}\left(\boldsymbol{C}_{\tilde{x}_{0}}(I)\right) \subseteq \Re$. This is an immediate consequence of Lemma 5.11 applied to $\Re^{N} \cap C\left(C_{\widetilde{x}_{0}}(I)\right) \times C_{\widetilde{x}_{0}}(I)$ acting on $\boldsymbol{O}_{2^{\prime}, 2}(\mathfrak{M}) / \boldsymbol{O}_{2^{\prime}}(\mathfrak{M})$. Hence, $\Re^{N}=\Re$, so $N_{\mathbb{\Theta}}\left(\mathfrak{T}_{1}^{*}\right) \subseteq \mathfrak{M}$. But $\mathfrak{I}_{1}^{*}=\widetilde{\mathfrak{I}}_{\mathfrak{1}} \cap \mathfrak{M}$ and $\tilde{\mathfrak{I}} \nsubseteq \mathfrak{M}$, so $\boldsymbol{N}_{\widetilde{x}}\left(\mathfrak{T}_{1}^{*}\right) \nsubseteq \mathfrak{M}$. This contradiction establishes our assertion.

Let $\mathscr{B}$ be the set of all subgroups $\mathscr{B}$ which are contained in $\boldsymbol{O}_{2^{\prime}, 2}(\mathfrak{M})$ and are also in $\mathscr{C}(\mathfrak{T})$ for some $S_{2}$-subgroup $\mathfrak{I}$ of $\mathfrak{M}$. Suppose $\mathfrak{B} \in \mathscr{P}$, and $\tilde{\mathfrak{I}}$ is a $S_{2}$-subgroup of $\mathfrak{M}$. If $\langle\boldsymbol{Z}(\widetilde{\mathfrak{T}}), \mathfrak{B}\rangle$ is a 2-group, then since $\boldsymbol{Z}(\tilde{\mathfrak{I}})$ maps into the center of $\boldsymbol{O}_{2^{\prime}, 2}(\mathfrak{M}) / \boldsymbol{O}_{2^{\prime}}(\mathfrak{M})$, it follows that $\langle\boldsymbol{Z}(\tilde{\mathfrak{I}}), \mathfrak{B}\rangle$ is abelian.

Let $\mathscr{J}$ be the set of involutions of $\boldsymbol{O}_{2^{\prime}, 2}(\mathfrak{M})$ which centralize at 
least one element of $\mathscr{B}$. We will show that for each $I$ in $\mathscr{J}, C_{\mathscr{\oplus}}(I) \subseteq \mathfrak{M}$. Suppose false. Among all elements of $\mathscr{J}$ whose centralizers are not contained in $\mathfrak{M}$, choose $I$ so that $\left|\boldsymbol{C}_{\mathscr{G}}(I)\right|_{2}$ is maximal. Let $\mathfrak{I}^{*}$ be a $S_{2}$-subgroup of $C_{\mathfrak{m}}(I)$. By our previous argument, $\mathfrak{I}^{*}$ is a $S_{2}$-subgroup of $C_{\Theta}(I)$. Let $\tilde{\mathfrak{I}}$ be a $S_{2}$-subgroup of $\mathfrak{M}$ which contains $\mathfrak{I}^{*}$ and choose

$$
\widetilde{Z} \in \Omega_{1}(\boldsymbol{Z}(\tilde{\mathfrak{I}}))^{\#} .
$$

Thus, $\langle I, \widetilde{Z}\rangle$ is a four-group.

Let $\mathfrak{I}_{1}^{*}=\mathfrak{I}^{*} \cap \boldsymbol{O}_{2^{\prime}, 2}\left(\boldsymbol{C}_{\circledast}(I)\right)$.

If $\subseteq$ is any solvable subgroup of $\mathscr{S}$ and if $\mathfrak{I}^{*}$ is a $S_{2}$-subgroup of $\mathfrak{S}$, then since $\mathfrak{I}_{1}^{*} \triangleleft \mathfrak{I}^{*}$, it follows from Lemma 5.11 that $\boldsymbol{O}_{2^{\prime}}(\mathfrak{S})$ contains every element of $\boldsymbol{U}_{\mathfrak{S}}\left(\mathfrak{I}_{1}^{*} ; 2^{\prime}\right)$.

Choose $N$ in $N\left(\mathfrak{T}_{1}^{*}\right) \cap C_{\mathscr{\leftrightarrow}}(I)$. Thus, $\mathfrak{I}_{1}^{*}$ normalizes $\Re^{N}$. Suppose $\Re^{N} \subseteq \mathfrak{M}$. Let $\widetilde{\Re}=C_{\Theta}(I) \cap \Re^{N}$. Thus, taking $\subseteq=C_{\Theta}(I)$, we get

$$
\tilde{\AA} \subseteq \boldsymbol{O}_{2^{\prime}}\left(C_{\mathfrak{s}}(I)\right) \text {. }
$$

By Lemma 3.7 of [20], it follows that $\tilde{\Re} \subseteq \Re$. Now $\left[\Re^{N}, I\right] \subseteq \Omega$ since $I \in \mathfrak{I}_{0} \subseteq \boldsymbol{O}_{2^{\prime}, 2}(\mathfrak{M})$. Hence, $\Re^{N}=\left(C_{G}(I) \cap \Re^{N}\right)\left[\Re^{N}, I\right] \subseteq \Re$, so $N \in \mathfrak{M}$. Since $C_{\Theta}(I)=\left(C_{\circledast}(I) \cap N_{\Theta}\left(\mathfrak{T}_{1}^{*}\right)\right) \cdot O_{2^{\prime}}\left(C_{\mathfrak{G}}(I)\right)$ and since $\mathfrak{T}^{*}$ contains an element of $\mathfrak{B}$, it follows that $C_{\mathscr{\Im}}(I) \subseteq \mathfrak{M}$. We may therefore assume that $\Re^{N} \nsubseteq \mathfrak{M}$.

Now $\AA^{N}=\left(\Re^{N} \cap \boldsymbol{C}(I)\right)\left(\Re^{N} \cap \boldsymbol{C}(\widetilde{Z})\right)\left(\Re^{N} \cap \boldsymbol{C}(\widetilde{Z} I)\right)$. Since $\Re^{N} \cap \boldsymbol{C}(I) \subseteq$ $\boldsymbol{O}_{2^{\prime}}(\boldsymbol{C}(I)) \subseteq \mathfrak{M}$, and since $\boldsymbol{C}_{\mathfrak{G}}(\widetilde{Z}) \subseteq \mathfrak{M}$, it follows that $\Re^{N} \cap \boldsymbol{C}(\widetilde{Z} I) \varsubsetneqq \mathfrak{M}$. Now $C(\widetilde{Z} I) \supseteq \mathfrak{I}^{*}$, so by maximality of $\left|\boldsymbol{C}_{\mathscr{\Theta}}(I)\right|_{2}$, it follows that $\mathfrak{I}^{*}$ is a $S_{2}$-subgroup of $C_{\Theta}(\widetilde{Z} I)$. Taking $\subseteq=C_{\Theta}(\widetilde{Z} I)$, we get

$$
\mathcal{R}^{N} \cap C(\widetilde{Z} I) \subseteq O_{2^{\prime}}(C(\widetilde{Z} I)) \subseteq \mathfrak{M}
$$

This contradiction shows that if $I \in \mathscr{F}$, then $C_{\circledast}(I) \subseteq \mathfrak{M}$.

Suppose now that $G \notin \mathfrak{M}$. We will show that $\mathscr{F} \cap \mathscr{J}^{G}=\varnothing$. Suppose false and $I \in \mathscr{J} \cap \mathscr{J}^{G}$. Then $C_{\mathfrak{M}}(I)$ and $\boldsymbol{C}_{\mathfrak{M}}{ }^{G}(I)$ both contain $S_{2}$-subgroups of $C_{\mathscr{\oplus}}(I)$, since $C_{\circledast}(I)=C_{\mathfrak{M}_{G}}(I)$. Thus, a $S_{2}$-subgroup of $C_{\mathfrak{n}}(I)$ is contained in some $S_{2}$-subgroup of $\mathfrak{M}^{G}$. Since each element of $\mathscr{F}$ is in $\mathfrak{M}$, it follows that $\mathfrak{M} \cap \mathfrak{M}^{G}$ contains a $S_{2}$-subgroup of $\mathbb{S}$. Since $\mathfrak{I} \in \mathscr{C}^{*}(\mathbb{S})$, we get $\mathfrak{M}=\mathfrak{M}^{G}$, against $G \notin \mathfrak{M}$.

We next show that if $G \notin \mathfrak{M}$, then $\mathfrak{I}_{0} \cap \mathfrak{M}^{G}$ is elementary. Suppose false and $T$ is an element of $\mathfrak{I}_{0}$ of order $4, T \in \mathfrak{M}^{G}$. Then $I=$ $T^{2} \in \boldsymbol{D}\left(\mathfrak{I}_{0}\right)$, so $I \in \mathscr{F}$. Let $\tilde{\mathfrak{I}}$ be a $S_{2}$-subgroup of $\mathfrak{M}^{G}$ which contains $T$ and let $\tilde{\mathfrak{I}}_{0}=\tilde{\mathfrak{I}} \cap \boldsymbol{O}_{2^{\prime}, 2}\left(\mathfrak{M}^{G}\right)$. Then $\tilde{\mathfrak{I}} \cap\langle T\rangle=1$, since $I$ centralizes every element of $\mathscr{U}(\tilde{\mathfrak{I}})$.

Let $\mathfrak{E}=\boldsymbol{C}(I) \cap \tilde{\mathfrak{I}}_{0}$. Then $[T, \mathfrak{E}] \subseteq \boldsymbol{O}_{2^{\prime}, 2}(\mathfrak{M})$, since $C(I) \subseteq \mathfrak{M}$. Since every element of $[T, \mathbb{E}]$ centralizes every element of $\mathscr{C}(\widetilde{\mathfrak{T}})$, it follows that $[T$, ( $]=1$. 
Now $T$ normalizes an element $\&$ of $\mathscr{S C C N}\left(\tilde{\mathfrak{I}}_{0}\right)$ and $\&$ contains an element of $\mathscr{U}(\tilde{\mathfrak{I}})$. Since $C_{\mathfrak{E}}(I)=C_{\mathfrak{E}}(T)$, it follows that $T$ centralizes (צ. Hence, $\mathfrak{\Im} \subseteq \mathbb{M}$. Since $\mathbb{F}$ contains an element of $\mathscr{U}(\tilde{\mathfrak{I}})$, it follows that $\Re \subseteq \mathfrak{M}^{G}$. Since $\Re \in h\left(\mathfrak{F} ; 2^{\prime}\right)$, it follows that $\mathfrak{R} \subseteq \boldsymbol{O}_{2^{\prime}}\left(\mathfrak{M}^{G}\right)=\Re^{G}$. This violates the assumption that $G \notin \mathfrak{M}$, so $\mathfrak{I}_{0} \cap \mathfrak{M}^{G}$ is elementary.

We rephrase the previous result in a more convenient form: if $\mathfrak{M}_{1}$ and $\mathfrak{M}_{2}$ are distinct conjugates of $\mathfrak{M}$, then $\boldsymbol{O}_{2^{\prime}, 2}\left(\mathfrak{M}_{1}\right) \cap \mathfrak{M}_{2}$ has elementary abelian $S_{2}$-subgroups.

We now choose an element $Z$ in $\Omega_{1}(\boldsymbol{Z}(\mathfrak{T}))^{\sharp}$. Suppose $Z^{G} \in \mathfrak{M}$. We will show that $G \in \mathfrak{M}$. Suppose false. We assume without loss of generality that $C_{\tilde{\Sigma}}\left(Z^{G}\right)$ is a $S_{2}$-subgroup of $C_{\mathfrak{n}}\left(Z^{G}\right)$. In particular, $Z^{G} \in \mathfrak{I}$, so $Z^{G}$ normalizes $\mathfrak{I}_{0}$.

Since $\boldsymbol{C}_{\mathscr{\leftrightarrow}}\left(Z^{G}\right) \subseteq \mathfrak{M}^{G}$, it follows that $\boldsymbol{C}_{\mathfrak{x}_{0}}\left(Z^{G}\right)$ is elementary.

Let $\mathfrak{B}$ be an element of $\mathscr{U}(\mathfrak{I})$ with $\mathfrak{B} \subseteq \mathfrak{I}_{0}$. Let $\widetilde{\mathfrak{I}}_{0}=\boldsymbol{C}_{\mathfrak{x}_{0}}(\mathfrak{B})$. If $C_{\widetilde{\mathfrak{z}}_{0}}\left(Z^{G}\right)$ is of order 2 , then $\left\langle Z^{G}, \widetilde{\mathfrak{I}}_{0}\right\rangle$ is of maximal class. Since $\mathfrak{B} \subseteq Z\left(\tilde{\mathfrak{I}}_{0}\right)$, it follows that $\tilde{\mathfrak{I}}_{0}=\mathfrak{B}$, and so $\mathfrak{I}_{0}$ is either a four-group or a dihedral group of order 8. Both possibilities are excluded since $2 \in \pi_{3}$. Hence, $C_{\widetilde{z}_{0}}\left(Z^{G}\right)$ is elementary of order at least 4 , and of course $Z \in \boldsymbol{C}_{\widetilde{\mathfrak{x}}_{0}}\left(Z^{G}\right)$.

Let $\mathfrak{I}^{*}$ be a $S_{2}$-subgroup of $\mathfrak{M}^{G}$ which contains $C_{\tilde{z}}\left(Z^{G}\right)$. Let

$$
\mathfrak{T}_{0}^{*}=\mathfrak{I}^{*} \cap \boldsymbol{O}_{2^{\prime}, 2}\left(\mathfrak{M}^{G}\right) \text {. }
$$

Let $\mathfrak{B}^{*} \in \mathscr{C}\left(\mathfrak{T}^{*}\right), \mathfrak{B}^{*} \subseteq \mathfrak{I}_{0}^{*}$ and let $\tilde{\mathfrak{T}}_{0}^{*}=C_{\mathfrak{x}_{0}^{*}}\left(\mathfrak{B}^{*}\right)$.

Choose $C$ in $\boldsymbol{C}_{\widetilde{x}_{0}}\left(Z^{G}\right)^{\sharp}$. Then $\left[\boldsymbol{C}_{\widetilde{\mathfrak{x}}_{0}^{*}}(C), \boldsymbol{C}_{\widetilde{\mathfrak{x}}_{0}}\left(Z^{G}\right)\right]$ is in

$$
\boldsymbol{O}_{2^{\prime}, 2}(\mathfrak{M}) \cap \boldsymbol{O}_{2^{\prime}, 2}\left(\mathfrak{M}^{G}\right)
$$

and being contained in $\mathfrak{I}^{\prime} \cap \mathfrak{T}^{* \prime}$, we have $\left[\boldsymbol{C}_{\widetilde{x}_{0}^{*}}(C), \boldsymbol{C}_{\widetilde{x}_{0}}\left(Z^{G}\right)\right]=1$. That is, all elements of $\boldsymbol{C}_{\widetilde{\mathfrak{x}}_{0}}\left(Z^{G}\right)^{\sharp}$ have the same fixed points on $\tilde{\mathfrak{I}}_{0}^{*}$. Also, $C_{\widetilde{x}_{0}}\left(Z^{G}\right)$ is faithfully represented on $\tilde{\mathfrak{I}}_{0}^{*}$ and $\boldsymbol{C}_{\widetilde{\mathfrak{x}}_{0}^{*}}(Z)$ is faithfully represented on $\tilde{\mathfrak{I}}_{0}$. This implies that $\left|\boldsymbol{C}_{\widetilde{\mathfrak{x}}_{0}^{*}}(Z)\right|=\left|\boldsymbol{C}_{\widetilde{\mathfrak{x}}_{0}}\left(Z^{G}\right)\right|=2^{a}$, with $a \geqq 2$. Let $\mathfrak{K}=\widetilde{\mathfrak{I}}_{0} \boldsymbol{C}_{\mathfrak{x}_{0}^{*}}(Z), \quad \mathfrak{U}=\left\langle\boldsymbol{C}_{\widetilde{\mathfrak{x}}_{0}^{*}}(Z), \boldsymbol{C}_{\widetilde{\mathfrak{x}}_{0}}\left(Z^{G}\right)\right\rangle=\mathfrak{A}_{1} \times \mathfrak{A}_{2}$, where $\mathfrak{A}_{1}=C_{\widetilde{\mathfrak{x}}_{0}^{*}}(Z), \mathfrak{A}_{2}=C_{\widetilde{\mathfrak{x}}_{0}}\left(Z^{G}\right)$. Since $a \geqq 2$, it follows that $\left|\widetilde{\mathfrak{I}}_{0}: \mathfrak{A}_{2}\right| \geqq 4$. Hence, it follows readily that $\boldsymbol{A}_{\mathfrak{f}}(\mathscr{C})$ contains a four-group, where $\mathscr{C}: \mathfrak{A} \supset \mathfrak{A}_{2} \supset 1$. Each element of $\boldsymbol{A}_{\mathfrak{y}}(\mathscr{C})^{\#}$ induces an isomorphism between $\mathfrak{U} / \mathfrak{A}_{2}$ and $\mathfrak{A}_{2}$. By symmetry, it follows that $\boldsymbol{A}_{\circledast}(\mathfrak{H})$ is nonsolvable. This contradiction shows that if $Z^{G} \in \mathfrak{M}$, then $G \in \mathfrak{M}$.

Now let $I$ be any involution of $\mathfrak{M}$. Let $\mathfrak{I}^{*}$ be a $S_{2}$-subgroup of $C_{\mathfrak{R}}(I)$. We may assume that $\mathfrak{T}^{*} \subseteq \mathfrak{T}$. If $\mathfrak{T}^{*}$ is not a $S_{2}$-subgroup of $C_{\mathscr{\Theta}}(I)$, choose $T$ in $N_{\mathscr{\Im}}\left(\mathfrak{T}^{*}\right) \cap \boldsymbol{C}_{\mathbb{S}}(I)-\mathfrak{M}$. Then $Z^{T} \in \mathfrak{M}$, so $T \in \mathfrak{M}$, an absurdity. Hence, $\mathfrak{I}^{*}$ is a $S_{2}$-subgroup of $C_{\circledast}(I)$. Since $C_{\Theta}(I) \cap \boldsymbol{O}_{2^{\prime}, 2}(\mathfrak{M})$ contains a four-subgroup whose 3 involutions are in $\mathscr{J}$, we have $\boldsymbol{O}_{2^{\prime}}\left(\boldsymbol{C}_{\circledast}(I)\right) \subseteq \mathfrak{M}$. Since $Z \in \boldsymbol{O}_{2^{\prime}, 2}\left(C_{\Theta}(I)\right)$, we get $\boldsymbol{C}_{\Theta}(I) \subseteq \mathfrak{M}$. This completes the proof. 
Theorem 10.8. Suppose $p \in \pi_{3}-\{2\}$ and $\mathscr{A}(p) \nsubseteq \mathscr{C l}^{*}(\mathrm{~S})$. Then

(i) $3 \in \pi_{4}$.

(ii) $2 \notin \pi_{4}$.

(iii) There are a prime $q \geqq 5$ and a subgroup $\mathfrak{B}$ of type $(q, q)$ such that $\mathfrak{B}$ does not centralize every element of $\boldsymbol{U}(\mathfrak{B} ; 2)$ and $\mathfrak{B}$ does not centralize every element of $И(\mathfrak{B} ; 3)$.

Proof. Let $\sigma$ be the equivalence class of $\pi_{3}-\{2\}$ under $\sim$ which contains $p$, and let $\pi=\sigma \cup \tau(\sigma)$. Let $\mathfrak{F}_{\mathcal{C}}$ be a $S_{\pi}$-subgroup of $\mathbb{S}$, which exists by Theorem 10.5. It is an easy consequence of Theorem 10.6 that for each $q$ in $\sigma, \mathscr{A}(q) \nsubseteq \mathscr{C}^{*}(\mathbb{S})$. For each set of primes $\pi_{0}$, let $\mathfrak{S}_{\pi_{0}}$ be a $S_{\pi_{0}}$-subgroup of $\mathfrak{S}_{2}$.

First, suppose that there is a prime $q$ in $\sigma$ such that the $S_{q^{-}}$ subgroup of $\boldsymbol{F}(\mathfrak{S})$ is noncyclic. In this case, let $\mathfrak{B}$ be a subgroup of $\boldsymbol{F}\left(\mathfrak{S}_{\mathcal{L}}\right)$ of type $(q, q)$ which is normal in $\mathfrak{S}_{q}$.

Let $\pi^{*}$ be the set of all primes $s \neq q$ such that $\mathfrak{B}$ does not centralize every element of $И(\mathfrak{B} ; s)$. If $\left|\pi^{*}\right| \leqq 1$, then by the argument of Theorem 0.24.1, it follows that $\left\langle\Re \mid \Re \in И\left(\mathfrak{S}_{q}\right)\right\rangle$ is a solvable $q^{\prime}$-group so by Theorem 10.7, $\mathscr{A}(q) \subseteq \mathscr{L}^{*}(\mathbb{S})$. This is not the case, so $\left|\pi^{*}\right| \geqq 2$. By definition of $\pi$, and by the transitivity theorem, it follows that $\pi \cap \pi^{*}=\varnothing$, and $\pi^{*} \cap\left(\pi_{1} \cup \pi_{2} \cup \pi_{3}-\{2\}\right)=\phi$. Suppose $s \in \pi^{*}, s \geqq 5$. Then $s \in \pi_{4}$. By Theorem 10.2 , $\mathscr{A}(s) \subseteq \mathscr{C}^{*}(\mathbb{S})$. It follows readily from Theorem 10.7 that $\mathscr{A}(q) \subseteq \mathscr{C}^{*}(\mathbb{S})$. This is not the case, so $\pi^{*} \subseteq\{2,3\}$. Hence, $\pi^{*}=\{2,3\}$. By construction, $3 \in \pi_{4}$. Thus, (i) and (iii) are satisfied.

Let $\mathfrak{P}_{0}$ be a maximal element of $И\left(\mathfrak{S}_{q} ; 2\right)$, and let $\mathfrak{\Omega}_{0}$ be a maximal element of $\boldsymbol{U}\left(\mathfrak{S}_{q} ; 3\right)$. Thus, $\mathfrak{B}$ is represented nontrivially on both $\mathfrak{P}_{0}$ and $\mathfrak{D}_{0}$. Let $\mathfrak{F}$ be an elementary subgroup of $\mathfrak{S}_{q}$ of order $q^{3}$ which contains $\mathfrak{B}$. We can choose a subgroup $\mathfrak{F}_{0}$ of $\mathfrak{F}$ of order $q^{2}$ with $\left[\boldsymbol{C}_{\mathfrak{F}_{0}}\left(\mathfrak{F}_{0}\right),[\mathfrak{F}] \neq 1\right.$, and we can choose a subgroup $\mathfrak{F}_{1}$ of $\mathbb{F}$ of order $q^{2}$

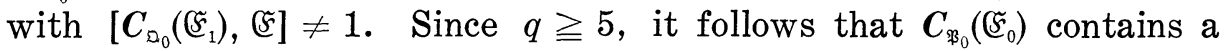
noncyclic abelian subgroup of order 8 , and $C_{\mathfrak{\Xi}_{0}}\left(\xi_{1}\right)$ contains an elementary subgroup of order 27.

Suppose $2 \in \pi_{4}$. Let $\mathfrak{F}^{*}=\mathbb{\mho}_{0} \cap \mathfrak{F}_{1} \neq 1$. Then $2 \sim 3$, as $\boldsymbol{C}_{\mathrm{G}}\left(\mathfrak{F}^{*}\right)$ exhibits. Thus, Theorems 8.1 and 9.1 imply that $(S)$ is isomorphic to either $S_{4}(3)$ or $E_{2}(3)$; both possibilities being excluded, we conclude that $2 \notin \pi_{4}$.

We may assume now that $\boldsymbol{F}\left(\mathfrak{S}_{2}\right) \cap \mathscr{S}_{\sigma}$ is cyclic. We will show that

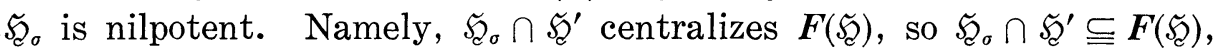
so $\mathfrak{S}_{\sigma} \cap \mathfrak{S}_{\mathcal{C}}^{\prime}$ is cyclic. In particular, $\mathfrak{S}_{\sigma}^{\prime}$ is cyclic. By Theorem 10.1, it follows that $\mathfrak{S}_{\sigma}$ is nilpotent.

Let $r$ be any prime $\geqq 5, r \notin \sigma$, and let $q \in \sigma$. We will show that every $q, r$-subgroup of $\mathbb{B}$ is $r$-closed. Suppose false. Thus, $r \| \boldsymbol{A}_{\mathbb{S}}(\mathfrak{\Omega}) \mid$ for some $q$-subgroup $\mathfrak{Q}$ of $\mathbb{6}$. It follows from Lemma 5.51 that 
$\mathfrak{S}_{q}$ contains a normal subgroup $\tilde{\mathcal{K}}_{q}$ such that $r \| A_{\Theta}\left(\tilde{\mathcal{F}}_{q}\right) \mid$. Let $\mathfrak{K}_{q}^{*}$ be a normal subgroup of $\mathfrak{S}_{q}$ maximal subject to $r|| \boldsymbol{A}_{\mathscr{\Theta}}\left(\mathfrak{S}_{q}^{*}\right) \mid$. Hence, $\mathfrak{S}_{q}^{*}$ contains every element of $\mathscr{S C E}_{\mathfrak{B}}\left(\mathscr{S}_{q}\right)$. Let $\Re_{0}$ be a $S_{r}$-subgroup of $\boldsymbol{N}_{\mathscr{\Theta}}\left(\mathfrak{S}_{q}^{*}\right)$ which is permutable with $\mathfrak{K}_{q}$ and let $\&$ be a maximal $q, r$ subgroup of $\mathbb{S}$ which contains $\mathfrak{S}_{q} \mathfrak{R}_{0}$. By the transitivity theorem, $\mathfrak{F}_{q} \cap \mathbb{R}^{\prime}$ centralizes $\boldsymbol{F}(\mathfrak{S})$, so $\mathfrak{S}_{q} \cap \mathfrak{R}^{\prime}$ is cyclic. This implies that $q \equiv 1$ $(\bmod r)$, and that $r \| S_{:} \mathscr{S}^{\prime} \mid$ for every solvable subgroup $\subseteq$ of $\mathbb{S}$ which contains \&. It follows that \&s satisfies $E_{q, r}$, so that \& is a $S_{q, r}$-subgroup of (8). Hence, $r \notin \pi_{3}$ since $r \notin \sigma$. Since $r \geqq 5$, it follows from Theorem 10.2 that $r \notin \pi_{4}$. Thus, $r \in \pi_{1} \cup \pi_{2}$ and (S) contains a normal subgroup of index $r$, against the simplicity of S. Hence, every $q, r$-subgroup of 13 is $r$-closed.

It is an immediate consequence of the preceding paragraph that $\mathfrak{S}_{\tau(\sigma)} \triangleleft \mathfrak{\mathfrak { F }}$.

By the transitivity theorem, $\left[\boldsymbol{F}\left(\mathfrak{S}_{2}\right), \mathfrak{S}_{\sigma}\right]$ is noncyclic. Choose $q$ so that the $S_{q}$-subgroup of $\left[\boldsymbol{F}(\mathfrak{C}), \mathscr{S}_{\sigma}\right]$ is noncyclic, and let $\mathfrak{B}$ be a subgroup of $\left[\boldsymbol{F}\left(\mathfrak{S}_{\mathcal{C}}\right), \mathfrak{S}_{\sigma}\right]$ of type $(q, q)$ which is normal in $\mathfrak{S}_{q}$.

Let $\pi^{*}$ be the set of primes $s \neq q$ such that $\mathfrak{B}$ does not centralize every element of $\boldsymbol{U}(\mathfrak{B} ; s)$. By the transitivity theorem, $\pi \cap \pi^{*}=\varnothing$. Also $\left|\pi^{*}\right| \geqq 2$, since otherwise $\mathscr{A}(p) \subseteq \mathscr{M}^{*}(\mathcal{S})$, as may be easily verified.

Suppose $s \in \pi^{*}, s$. Choose $q_{1}$ in $\sigma$. Since $\mathscr{S}_{q_{1}} \cap \boldsymbol{C}\left(\boldsymbol{O}_{q}\left(\mathfrak{S}_{e}\right)\right)$ is noncyclic, it follows that $\mathfrak{S}_{q_{1}}$ normalizes a $S_{s}$-subgroup $\subseteq$ of (B). Suppose $\mathfrak{S}_{q_{1}}$ does not centralize $\mathfrak{S}$. Clearly, $s \notin \pi_{3}$, since $s \notin \sigma$. Also, $s \notin \pi_{4}$, since $\mathscr{A}\left(q_{1}\right) \nsubseteq \mathscr{M}^{*}(\mathbb{S})$. Hence, $s \in \pi_{1} \cup \pi_{2}$, so $s \in \tau(\sigma)$. This violates $\pi^{*} \cap \pi=\varnothing$, and so $\mathfrak{S}_{q_{1}}$ centralizes $\mathfrak{S}$. It follows that $\mathfrak{K}_{\sigma}$ centralizes a $S_{s}$-subgroup of $\mathbb{S}$, call it $\widetilde{S}$. This implies that $\left[\boldsymbol{O}_{q}\left(\mathfrak{S}_{\mathcal{E}}\right), \mathfrak{S}_{\mathcal{C}}\right]$ centralizes every element of $И\left(O_{q}(\mathfrak{S}) ; s\right)$. This violates our choice of $\mathfrak{B}$. Hence $\pi^{*} \subseteq\{2,3\}$, so $\pi^{*}=\{2,3\}$.

Since $\mathscr{S}_{p} \cap \boldsymbol{C}\left(\boldsymbol{O}_{q}\left(\mathfrak{S}_{c}\right)\right)$ is noncyclic, it follows that $3 \in \pi_{4}$. If $2 \notin \pi_{4}$, we are done, so suppose by way of contradiction that $2 \in \pi_{4}$. Since $\mathfrak{S}_{p} \cap \boldsymbol{C}\left(\boldsymbol{O}_{q}\left(\mathfrak{S}_{\mathcal{C}}\right)\right)$ is noncyclic, it follows that $\mathfrak{S}_{p}$ does not centralize every element of $\boldsymbol{U}\left(\mathfrak{S}_{p} ; 2\right)$ and $\mathfrak{S}_{p}$ does not centralize every element of $\boldsymbol{U}\left(\mathfrak{S}_{p} ; 3\right)$. Hence, 2 3, against Theorems 8.1 and 9.1. The proof is complete.

Theorem 10.9. Suppose $\mathfrak{I}$ is a $S_{2}$-subgroup of $\mathbb{B}$ and $\mathfrak{M}$ is solvable subgroup of (S) such that

(a) $N_{\circledast}(\mathfrak{I}) \subseteq \mathfrak{M}$,

(b) $\mathfrak{M}$ contains the centralizer of each of its involutions. Let $p$ be an odd prime, $p \in \pi_{3} \cup \pi_{4}$. Suppose $\mathfrak{M}$ contains an element of $\mathscr{A}(p)$. Then $\mathscr{A}(p) \subseteq \mathscr{C}^{*}(\mathbb{S})$.

Proof. It is easy to verify that if $G \notin \mathfrak{M}$, then $\left|\mathfrak{M} \cap \mathfrak{M}^{a}\right|$ is odd. By Lemma 5.35, $i(\mathbb{S})=i(\mathfrak{M})=1$. Furthermore, as in the first part. 
of the proof of Theorem 10.4, if $\subseteq$ is a solvable subgroup of $\mathbb{S}$ and $\subseteq \cap \mathfrak{M}$ contains a four-group then $\subseteq \subseteq \mathfrak{M}$.

Let $\mathfrak{P}$ be a $S_{p}$-subgroup of $\mathfrak{M}$ permutable with $\mathfrak{I}$, and let $\mathfrak{P}^{*}$ be a $S_{p}$-subgroup of (S) which contains $\mathfrak{P}$. Let $3=\Omega_{1}(\mathfrak{T})=\Omega_{1}(Z(\mathfrak{T})$ ). By Lemma 5.40, $\boldsymbol{O}_{p}(\mathfrak{P} \mathfrak{T}) 3 \triangleleft \mathfrak{P T}$. Set $\mathbb{R}=\mathfrak{P T}$.

Suppose $\boldsymbol{O}_{2}(\mathbb{R}) \neq 1$. In this case, $\boldsymbol{O}_{p^{\prime}}(\mathfrak{M})$ is of even order, so $3 \subseteq$ $\boldsymbol{O}_{p^{\prime}}(\mathfrak{M})$, so that $3 \triangleleft \mathfrak{R}$. Let $i$ be the smallest integer such that $\mathfrak{P}$ contains a subgroup $\mathfrak{B}$ of type $(p, p)$ with $\mathfrak{B} \in \mathscr{A}_{i}(p)$. We can then choose $B$ in $\mathfrak{B}^{\sharp}$ with $C_{8}(B)$ noncyclic. Then $C_{\mathbb{S}}(B) \subseteq \mathfrak{M}$, so $i=1$. Hence, $\mathfrak{P}$ contains an element of $\mathscr{S C C N}_{3}(p)$, and it follows that $\mathfrak{P}$ is a $S_{p}$ subgroup of $\mathbb{S}$. We can then choose a subgroup $\mathbb{F}$ of $\mathfrak{P}$ of type $(p, p)$ such that $C_{3}(\mathfrak{S})$ is noncyclic. Hence $C_{\mathfrak{\Theta}}(E) \subseteq \mathfrak{M}$ for all $E$ in $\mathfrak{F}^{\sharp}$. Hence, every element of $U(\mathfrak{B})$ is contained in $\mathfrak{M}$. By Theorem 10.6, we are done.

For the remainder of the proof, we may assume that $\boldsymbol{O}_{2}(\mathbb{R})=1$. Let $\tilde{\mathfrak{L}}=\mathfrak{P} \mathfrak{Z}$, so that $\tilde{\mathfrak{\Omega}}$ is a group. By a result of Glauberman [17], $Z(J(\mathfrak{P})) \triangleleft \widetilde{\mathfrak{L}}$, so we get that

$$
N_{\circledast}(Z(J(\mathfrak{P}))) \subseteq \mathfrak{M}, \quad N_{\circledast}(Z(J(\mathfrak{P}))) \in \mathscr{C l}^{*}(\mathbb{S}) .
$$

In particular

$$
\mathfrak{F} \text { is a } S_{p} \text {-subgroup of } \mathfrak{B}, N(\mathfrak{P}) \subseteq \mathfrak{M} \text {. }
$$

Now (10.5) and (10.6) imply that

(10.7) $\mathfrak{M}$ is the only conjugate of $\mathfrak{M}$ which contains $\mathfrak{P}$.

Let $\mathfrak{O}$ be a maximal element of $И(\mathfrak{P} ; q)$ for some $q \neq p$. By the transitivity theorem, which may be applied since $p \notin \pi_{1} \cup \pi_{2}$, it follows that $N_{\circledast}(\mathfrak{\Omega})$ contains a four-group. Hence by $(10.7)$, we get

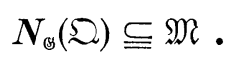

In particular, every element of $И(\mathfrak{P})$ is contained in $\mathfrak{M}$. If $p \in \pi_{3}$, we are done by Theorem 10.6. So suppose $p \in \pi_{4}$.

$$
\begin{aligned}
& \text { Let } \mathscr{A}_{i}^{*}(\mathfrak{P})=\{\mathfrak{X} \mid \mathfrak{A} \subseteq \mathfrak{P}, \mathfrak{U} \text { contains an element of } \\
& \left.\mathscr{A}_{i}\left(\mathfrak{S}^{M}\right) \text { for some } M \text { in } \mathfrak{M} .\right\}
\end{aligned}
$$

Let $i$ be the smallest integer such that $\mathscr{A}_{i}^{*}\left(\mathfrak{F}_{3}\right) \nsubseteq \mathscr{l l}^{*}(\mathbb{S})$, and choose $\mathfrak{A} \in \mathscr{A}_{i}^{*}(\mathfrak{S})-\mathscr{C l}^{*}(\mathbb{S})$ of maximal order. Let $\subseteq$ be a solvable subgroup of $\mathbb{S}$ of minimal order subject to $\mathfrak{U} \subseteq \subseteq, \subseteq \nsubseteq \mathfrak{M}$. Then $\subseteq=$ $\mathfrak{X} \Omega$, where $\cong$ is a $q$-group for some $q \neq p$. The minimality of $i$ guarantees that $\boldsymbol{O}_{q}(\subseteq) \subseteq \mathfrak{M}$. Suppose $q \neq 2$. Then

$$
\mathfrak{S}=O_{q}(\mathfrak{S}) \cdot N_{\Im}(Z(J(\mathfrak{U})))
$$


by the result of Glauberman [17], so $\subseteq \subseteq \mathfrak{M}$. Hence $q=2$. Suppose

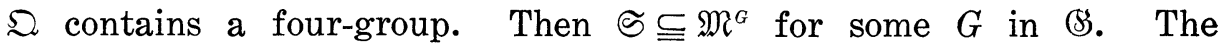
maximality of $\mathfrak{A}$ guarantees that $\mathfrak{N}$ is a $S_{p}$-subgroup of $\mathfrak{M}^{G}$. Thus $\mathfrak{M}^{\alpha}=\mathfrak{M}$ by (10.7). We may therefore assume that $\mathfrak{Q}$ contains no four-group. In this case, the involution $I$ of $\mathfrak{Q}$ normalizes $\mathfrak{A}$, and the maximality of $\mathfrak{U}$ gives $I \in \mathfrak{M}$. Hence $\mathfrak{C} \subseteq \boldsymbol{C}_{\oplus}(I) \subseteq \mathfrak{M}$, so $\subseteq \subseteq \mathfrak{M}$. The proof is complete.

11. The case $2 \in \pi_{2}$. Except for Lemmas 11.1 and 11.2, all results in this section are proved on the hypothesis that $2 \in \pi_{2}$.

The following notation will be used throughout this section. $\mathfrak{I}$ is a $S_{2}$-subgroup of $\left(\mathcal{B}, 3=\Omega_{1}(Z(\mathfrak{I}))\right.$. In case $|3|=2$, we set $3=\langle Z\rangle$. In case $\mathscr{C}(\mathfrak{I}) \neq \varnothing$, we choose a fixed element $\mathfrak{W}$ of $\mathscr{L}(\mathfrak{I})$ and let $\mathfrak{I}_{0}=C_{\mathfrak{z}}(\mathfrak{W})$. In case $|3|=2$ and $\mathscr{U}(\mathfrak{I}) \neq \varnothing$, we choose $I$ in $\mathfrak{W}-3$, and observe that $I \sim I Z$. Let $\Re=N_{\oplus}(3)$, let $\mathfrak{Q}$ be a $S_{2^{\prime}}$-subgroup of $\mathfrak{R}$, let $\Re=N_{\mathfrak{z}}(\mathfrak{\Omega})$, and let $\mathfrak{\mathcal { E }}=\mathfrak{I} \cap \boldsymbol{O}_{2^{\prime}, 2}(\mathfrak{R})$. Let $\mathfrak{\Omega}_{3}$ be a $S_{3}$-subgroup of $\mathfrak{R}$ permutable with $\mathfrak{T}$. Let $\overline{\mathfrak{N}}=\mathfrak{R} / \boldsymbol{O}_{2},(\mathfrak{R})$, and for any subset $\mathfrak{S}$ of $\mathfrak{R}$, let $\bar{\varsigma}$ be the image of $\subseteq$ in $\tilde{\mathfrak{R}}$. Further notation will be used for various special cases, but the preceding notation is always used.

Notice that if $\mathfrak{U}, \mathfrak{B}$ are 2 -subgroups of $\mathfrak{R}$, then $\mathfrak{U}$ and $\mathfrak{B}$ are conjugate in $\mathfrak{N}$ if and only if $\overline{\mathfrak{A}}$ and $\overline{\mathfrak{B}}$ are conjugate in $\overline{\mathfrak{A}}$. Finally, $\overline{\mathfrak{S}}=\boldsymbol{O}_{2}(\overline{\mathfrak{\Re}})$ and $\boldsymbol{C}_{\overline{\mathfrak{R}}}(\overline{\mathfrak{F}})=\boldsymbol{Z}(\overline{\mathfrak{F}}) \supseteqq \boldsymbol{Z}(\overline{\mathfrak{I}})=\overline{\boldsymbol{Z}(\mathfrak{I})}$.

A result of Glauberman is quite helpful [16]. Since (S) is simple, Glauberman's result implies that

$\mathfrak{I}$ has no weakly closed subgroup of order 2 .

In this section, the groups of symplectic type appear frequently (cf. Definition 2.4 and remark).

We begin with some elementary remarks. Suppose $p$ is a prime and $\mathfrak{A}, \mathfrak{B}$ are $p$-subgroups of a group $\mathbb{Z}$ and $\mathfrak{U} \subseteq N(\mathfrak{B}), \mathfrak{B} \subseteq N(\mathfrak{Z})$. Let $\mathfrak{Q}_{p}$ be a $S_{p}$-subgroup of $N(\mathfrak{Q}) \cap N(\mathfrak{B})$ and suppose that $\mathfrak{Q}_{p}$ is not a $S_{p}$-subgroup of $N(\mathfrak{B})$. Let $\mathbb{Q}_{p}^{*}$ be a $S_{p}$-subgroup of $N\left(\mathbb{R}_{p}\right) \cap$ $N(\mathfrak{B})$, so that $\mathfrak{Q}_{p}^{*} \supset \mathfrak{Q}_{p}$. Since $\mathfrak{Q}_{p}$ is a $S_{p}$-subgroup of $N(\mathfrak{U}) \cap N(\mathfrak{B})$, no element of $\Omega_{p}^{*}-\mathfrak{Q}_{p}$ normalizes $\mathfrak{X}$. In particular, $\mathfrak{U}$ is not characteristic in any subgroup of $\mathscr{R}_{p}$ which is weakly closed in $\mathbb{R}_{p}$ with respect to 2 . We record this.

Lemma 11.1. Suppose $\mathfrak{Z}, \mathfrak{B}$ are p-subgroups of a group $\mathbb{R}, \mathfrak{U} \subseteq$ $\boldsymbol{N}(\mathfrak{B}), \mathfrak{B} \cong \boldsymbol{N}(\mathfrak{i})$ and $\mathfrak{L}_{p}$ is a $S_{p}$-subgroup of $N(\mathfrak{I}) \cap \boldsymbol{N}(\mathfrak{B})$. Suppose also that $\mathfrak{R}_{p}$ is not a $S_{p}$-subgroup of $\boldsymbol{N}(\mathfrak{B})$. Then $\mathfrak{i}$ is not characteristic in any weakly closed subgroup of $\mathbb{R}_{p}$.

REMARK. In this section, this lemma will be used frequently in case $|\mathfrak{X}|=|\mathfrak{B}|=2$. 
We need an application of (11.1) and Lemma 11.1.

Lemma 11.2. Suppose $\mathfrak{R}_{2}$ is a $S_{2}$-subgroup of the group $\mathbb{R}$ and

(a) $\left|\boldsymbol{D}\left(\Omega_{2}\right)\right|=2$,

(b) $\Omega_{2}$ has no elementary abelian subgroup of index 2. Then $\boldsymbol{D}\left(\Omega_{2}\right) \subseteq Z\left(\Omega \bmod \boldsymbol{O}_{2^{\prime}}(\mathbb{R})\right)$.

Proof. Let $Z$ be the generator for $\boldsymbol{D}\left(\mathfrak{Q}_{2}\right)$, and let $I$ be an involution of $\Omega_{2}-D\left(\Omega_{2}\right)$. By (11.1), it suffices to show that $I$ and $Z$ are not $\&$-conjugate. Let $\mathfrak{R}=\boldsymbol{C}_{\Omega}(Z)$, and let $\mathfrak{P}$ be a $S_{2}$-subgroup of $\mathfrak{R} \cap$ $C(I)$. Choose $N$ in $\mathfrak{R}$ such that $\mathfrak{P}^{N}=\mathfrak{I} \subseteq \mathbb{R}_{2}$. Let $J=I^{N} \in \mathfrak{T}$. It suffices to show that $J$ and $Z$ are not \&-conjugate. In any case, $J \neq Z$, since $\mathfrak{N}$ centralizes $Z$. If $J \in Z\left(\Omega_{2}\right)$, then $J_{\widetilde{\mathfrak{Z}}} Z$ if and only if $J_{N\left(\widetilde{\Omega}_{2}\right)} Z$. Since $\langle Z\rangle$ char $N\left(\mathbb{Q}_{2}\right), J$ and $Z$ are not $\&$-conjugate in this case. We may assume that $J \notin Z\left(\mathbb{R}_{2}\right)$. Hence, $\left|\mathbb{R}_{2}: \mathfrak{I}\right|=2$, since $\left|\boldsymbol{D}\left(\mathbb{\Omega}_{2}\right)\right|=2$. Since $\mathfrak{I}$ is not elementary by assumption (b), we get $\boldsymbol{D}(\mathfrak{T})=\boldsymbol{D}\left(\mathbb{R}_{2}\right)=$ $\langle Z\rangle$, so by Lemma 11.1, $J$ and $Z$ are not $\mathcal{L}$-conjugate.

We now turn to our group (S).

Lemma 11.3. Suppose $\mathfrak{X}$ is a normal subgroup of $\mathfrak{I}$ of symplectic type and width $\geqq 2$. Then

(a) $\mathfrak{U}$ is extra special of order $2^{5}$.

(b) $\mathfrak{I} / \mathfrak{X}$ is faithfully represented on $\mathfrak{X} / \boldsymbol{D}(\mathfrak{Q})$.

Proof. Let $w$ be the width of $\mathfrak{U}$. As $\mathfrak{U}$ is of symplectic type, $\mathfrak{A}$ is the central product of $\mathfrak{N}_{0}$ and $\mathfrak{N}_{1}$ where $\mathfrak{U}_{0}$ is extra special of width $w$ and $\mathfrak{A}_{1}$ is either cyclic or of maximal class. Thus, $\boldsymbol{D}(\mathfrak{Z})=$ $\mathfrak{A}_{0}^{\prime} \boldsymbol{D}\left(\mathfrak{U}_{1}\right)$ and so $\Omega_{2}(\boldsymbol{D}(\mathfrak{H}))$ is cyclic of order at most 4 . Also, $\boldsymbol{C}_{\mathfrak{r}}\left(\Omega_{2}(\boldsymbol{D}(\mathfrak{I}))\right)$ is of class 2 , so that $\mathfrak{X}^{*}=\Omega_{2}\left(\boldsymbol{C}_{\mathfrak{H}}\left(\Omega_{2}(\boldsymbol{D}(\mathfrak{U}))\right)\right)$ is a characteristic subgroup of $\mathfrak{A}$ which is the central product of $\mathfrak{U}_{0}$ and a cyclic group of order $2^{1+e}$, where $e=0$ if $\left|\mathfrak{A}_{1}\right| \leqq 2$ and $e=1$ otherwise. By Lemma 5.23, some element $\mathfrak{B}$ of $\mathscr{S} \mathfrak{C} \mathscr{N}\left(\mathfrak{Q}^{*}\right)$ is normal in $\mathfrak{I}$. Hence $|\mathfrak{B}|=2^{1+w+e}$, so $\left|\Omega_{1}(\mathfrak{B})\right| \geqq 2^{e+w}$, since $|\boldsymbol{D}(\mathfrak{B})| \leqq 2$. As $\mathscr{S P C}_{3}(\mathfrak{I})=\varnothing$, we have $e+w \leqq 2$. Hence, $e=0, w=2$, so that $\mathfrak{A}=\mathfrak{U}^{*}=\mathfrak{A}_{\hat{s}}$.

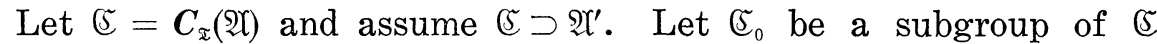
of order 4 which is normal in $\mathfrak{I}$. Then $\left|\mathfrak{C}_{0} \mathfrak{B}\right|=2^{4}$ and $\boldsymbol{D}\left(\mathfrak{C}_{0} \mathfrak{B}\right) \subseteq \mathfrak{U}$; so $m\left(\mathfrak{S}_{0} \mathfrak{B}\right) \geqq 3$, against $\mathscr{S} \mathscr{C} \mathscr{N}_{3}(\mathfrak{T})=\varnothing$. Hence, $\boldsymbol{C}_{\mathfrak{\Sigma}}(\mathfrak{U})=\mathfrak{U}^{\prime}$.

Suppose $X$ in $\mathfrak{I}$ centralizes $\mathfrak{U} / \mathfrak{I}^{\prime}$. Then $X$ induces an inner automorphism of $\mathfrak{A}$, so $X \in \mathfrak{A}$. Then proof is complete.

LEMma 11.4. (i) If $\mathscr{\mathscr { C }}(\mathfrak{I})=\varnothing$, then one of the following holds:

(a) $\mathfrak{I}$ is dihedral of order $\geqq 16$,

(b) $\mathfrak{I}=g p\left\langle A, B \mid A^{8}=B^{2}=1, B A B=A^{3}\right\rangle$, and $\overline{\mathfrak{N}}$ is isomorphic 
to the centralizer of an involution of $L_{3}(3)$.

(ii) If $\mathscr{U}(\mathfrak{T})$ has more than one element, then $\mathfrak{I}$ is dihedral of order 8.

Proof. (i) By the results of [9], $\mathfrak{T}$ is not a generalized quaternion group. The hypothesis $\mathscr{C}(\mathfrak{T})=\varnothing$ is equivalent to the hypothesis that every normal abelian subgroup of $\mathfrak{I}$ is cyclic, so either (i) (a) holds or

$$
T=g p\left\langle A, B \mid A^{2^{n}}=B^{2}=1, B A B=A^{-1+2^{n-1}}\right\rangle \quad n \geqq 3 .
$$

In the latter case, set $\mathfrak{I}^{*}=\left\langle A^{2}, B\right\rangle$. Then $\mathfrak{I}^{*}$ is a dihedral group and $\mathfrak{I}^{*}=\Omega_{1}(\mathfrak{I})$. More explicitly, every element of $\mathfrak{I}-\mathfrak{I}^{*}$ is a root of $Z$. As $(B)$ is simple, we can choose $G$ in $(S)$ so that $\mathfrak{I} \cap \mathfrak{I}^{* G} \nsubseteq \mathfrak{T}^{*}$. Choose $X$ in $\mathfrak{I} \cap \mathfrak{I}^{* G}-\mathfrak{T}^{*}$. Since $X \notin \mathfrak{I}^{*}, Z$ is the involution of $\langle X\rangle$. Since $X^{2} \in\left\langle A^{G}\right\rangle, Z^{G}$ is the involution of $\langle X\rangle$; that is, $Z=Z^{G}, G \in \mathfrak{N}$. Hence $\mathfrak{N}$ has no normal 2-complement. Application of Lemma 5.27 to $\overline{\mathfrak{F}}$ implies that $\overline{\mathfrak{F}}$ is a quaternion group. Hence $n=3$ and $\mathfrak{I}$ has the required shape. Since $B \notin \mathscr{S}$, it follows from Lemma 5.35 that $\bar{B}$ inverts a $S_{3}$-subgroup of $\overline{\mathfrak{A}}$, which we may assume is $\overline{\mathfrak{D}}$. These conditions determine the isomorphism class of $\overline{\mathfrak{R}}$, and it follows from direct inspection of $S L(3,3)$ that (i) (b) holds.

(ii) Suppose $\mathfrak{W}$ and $\mathfrak{W} *$ are distinct elements of $\mathscr{U}(\mathfrak{Z})$. Let $\mathfrak{D}=\mathfrak{B W}^{*}$. Since $2 \in \pi_{2}$, $\mathfrak{D}$ is a normal dihedral subgroup of $\mathfrak{I}$ of order 8 . Let $\mathfrak{E}=C_{\mathfrak{z}}(\mathfrak{D})$. Since $\mathfrak{W}$ and $\mathfrak{S}^{*}$ are normal in $\mathfrak{I}$, it follows that $\mathfrak{I}$ is the central product of $\mathbb{C}$ and $\mathfrak{D}$. Since $2 \in \pi_{2}$, every normal abelian subgroup of $\mathbb{E}$ is cyclic. Suppose by way of contradiction that $\mathfrak{D} \subset \mathfrak{I}$. In this case $|\mathfrak{S}| \geqq 4$.

Choose $G$ in (S) such that $Z_{1}=Z^{G} \in \mathfrak{I}, Z_{1} \neq Z ; Z_{1}$ is available by (11.1). Since $\boldsymbol{D}(\mathfrak{T})$ is cyclic, $Z_{1} \notin \boldsymbol{D}(\mathfrak{T})$. Let $\mathfrak{F}=C_{\mathfrak{x}}\left(Z_{1}\right)$, and let $\mathfrak{T}^{*}$ be a $S_{2}$-subgroup of $C_{\Theta}\left(Z_{1}\right)$ which contains $\&$, so that

$$
\left\langle Z_{1}\right\rangle=\Omega_{1}\left(\boldsymbol{Z}\left(\mathfrak{I}^{*}\right)\right)=\Omega_{1}\left(\boldsymbol{D}\left(\mathfrak{T}^{*}\right)\right) \text {. }
$$

If $\mathbb{F}$ is not elementary, then $Z \in \boldsymbol{D}(\mathfrak{S}) \subseteq \boldsymbol{D}\left(\mathfrak{T}^{*}\right)$ so that $Z=Z_{1}$. As this is not the case, $\mathbb{F}$ is elementary. Now $Z_{1}=C D, C \in \mathbb{E}, D \in \mathfrak{D}$. Since $\mathbb{C}$ and $\mathfrak{D}$ commute elementwise, we have

$$
C Z_{1}=C^{2} D=C D C=Z_{1} C,
$$

so that $C$ and $D$ commute with $Z_{1}$. Hence, $C^{2}=D^{2}=1$. If $C \in 3$, then $Z_{1}$ centralizes $\mathbb{\varepsilon}$, so that $Z \in D(\mathfrak{E}) \leqq D(\&)=1$. Hence, $C \neq 1, Z$. If $D \in 3$, then $\mathfrak{D}$ centralizes $Z_{1}$, so $Z \in \boldsymbol{D}(\mathfrak{D}) \subseteq \boldsymbol{D}(\mathfrak{F})=1$. Hence $D \neq$ $1, Z$.

Since (5 has more than one involution, $\sqrt{5}$ is of maximal class and order $\geqq 16$. Let $\mathfrak{\Im}_{0}=\left\langle C_{0}\right\rangle$ be the unique (cyclic) normal subgroup 
of $\mathbb{E}$ of order 4. Thus, $C$ inverts $\mathfrak{E}_{0}$. Let $\mathfrak{D}_{0}=\left\langle D_{0}\right\rangle$ be the unique cyclic subgroup of $D$ of order 4 . Thus, $D$ inverts $\mathscr{D}_{0}$. Also $C_{0}^{2}=$ $D_{0}^{2}=Z$. Hence, we compute that $\left(C_{0} D_{0}\right)^{-1} Z_{1} C_{0} D_{0}=Z_{1}, C_{0} D_{0} \in$ \&. Since (5) is elementary, $C_{0} D_{0}$ centralizes $C$. This is not the case since

$$
C^{-1} C_{0} D_{0} C=C^{-1} C_{0} C D_{0}=C_{0} Z D_{0}=C_{0} D_{0} Z \text {. }
$$

The proof is complete.

For each prime $p$, let $\mathscr{L}(p)=\{\mathbb{L} \mid \mathbb{R}$ is a 2-subgroup of $\mathbb{B}$ and $\left.p \| \boldsymbol{A}_{\Theta}(\mathbb{R}) \mid\right\}$. hold:

Lemma 11.6. Suppose $p \geqq 5$ and $\mathscr{L}(p) \neq \varnothing$. Then the following

(i) The image of $\boldsymbol{C}_{\mathfrak{G}}(Z) \cap N(\mathfrak{I})$ in $N(\mathfrak{T}) / \boldsymbol{O}_{2^{\prime}}(\boldsymbol{N}(\mathfrak{T})$ ) is isomorphic to the centralizer of an involution of $U_{3}(4)$.

(ii) $S_{2^{\prime}}$-subgroups of $N(\mathfrak{I}) / O_{2^{\prime}}(N(\mathfrak{T}))$ are of order 15 .

(iii) $i(\mathbb{S})=1$.

Proof. Choose $\mathbb{Q}$ in $\mathscr{L}(p)$ with $|N(\mathbb{R})|_{2}$ maximal, and with this restriction, maximize $\mathbb{2}$. Let $\mathfrak{M}$ be a $S_{2, p}$-subgroup of $N(\mathbb{R})$, let $\mathfrak{I}^{*}$ be a $S_{2}$-subgroup of $\mathfrak{M}$ and let $\mathfrak{M}_{p}$ be a $S_{p}$-subgroup of $\boldsymbol{O}_{p, 2, p}(\mathfrak{M})$. We assume without loss of generality that $\mathfrak{I}^{*} \subseteq \mathfrak{T}$.

Clearly, $\mathfrak{L}=\boldsymbol{O}_{p, 2}(\mathfrak{M}) \cap \mathfrak{I}^{*}$. Hence, $\boldsymbol{Z}(\mathfrak{I}) \subseteq \boldsymbol{Z}\left(\mathfrak{T}^{*}\right)$. By Theorem 1 of [43] applied to $\mathfrak{M}$, either $\boldsymbol{C}_{\mathfrak{M}}\left(\boldsymbol{Z}\left(\mathfrak{T}^{*}\right)\right)$ or $\boldsymbol{N}_{\mathfrak{n}}\left(\boldsymbol{J}\left(\mathfrak{I}^{*}\right)\right)$ has no normal 2-complement. Hence, $\mathfrak{I}=\mathfrak{I} *$.

Since $p>3$ and $\mathcal{S C C}_{\mathfrak{C}}(\mathfrak{I})=\varnothing, \mathfrak{M}_{p}$ centralizes every characteristic abelian subgroup of $\left[\mathfrak{M}_{p}, \mathfrak{\Omega}\right]=\Omega_{0}$. By Lemma 5.17, $\mathfrak{\Omega}_{0}$ is special.

First, suppose $\boldsymbol{Z}\left(\Omega_{0}\right)$ is of order 2 , so that $\Omega_{0}$ is extra special. Since $p>3$, the width of $\mathbb{R}_{0}$ is at least 2. By Lemma 11.3(a), $\left|\Omega_{0}\right|=$ $2^{5}$. Since $\mathfrak{R}_{0} / D\left(\Omega_{0}\right)$ is a chief factor of $\mathfrak{M}$, it follows that $\mathbb{Q}$ centralizes $\mathfrak{\Omega}_{0} / \boldsymbol{D}\left(\mathfrak{R}_{0}\right)$. Hence $\mathfrak{Q}=\mathfrak{\Omega}_{0}$ by Lemma $11.3(\mathrm{~b})$. Since $\mathfrak{M}_{p} / \boldsymbol{O}_{p}(\mathfrak{M})$ is of order $5, \mathfrak{I} / \Omega$ is cyclic of order $\leqq 4$.

Since $\mathfrak{M} \cong \mathfrak{R}$, it follows that $\mathbb{L}=\mathfrak{S}$, and that $S_{2^{\prime}}$-subgroups of $\overline{\mathfrak{R}}$ are of order 5 .

By (11.1), there is $Z_{1}=Z^{a} \in \mathfrak{I}, Z_{1} \neq Z$. Suppose $Z_{1} \in \mathfrak{S}$. Since $\mathfrak{M}$ permutes transitively the non central involutions of $\mathfrak{S}$, we may assume that $Z_{1}=I$. In this case $\boldsymbol{A}_{\mathscr{G}}\left(\mathfrak{I}_{0}\right)$ contains a subgroup $\langle A\rangle$ which permutes $\mathfrak{W}^{\sharp}$ transitively. Since $Z \in \mathfrak{I}_{0}^{\prime}$, so also $I \in \mathfrak{T}_{0}^{\prime}$. Hence, $\mathfrak{S} \subset \mathfrak{I}$. Since $\mathfrak{I} / \mathfrak{S}$ is cyclic, it follows that $\left(\mathfrak{I}_{0} \cap \mathfrak{F}\right)^{A} \cap \mathfrak{S}$ properly contains $\mathfrak{W}$. Hence $Z^{A} \in \mathfrak{W}-3$ and $\mathfrak{W}=\Omega_{1}\left(C_{\mathfrak{F}}(\mathfrak{W})\right)$, since $C_{\mathfrak{F}}(\mathfrak{W})$ is the direct product of $\langle I\rangle$ and a quaternion group. Hence $\left(\mathfrak{I}_{0} \cap \mathfrak{S}^{A} \cap \cap \mathfrak{F}\right.$ contains an element $L$ of order 4 . Hence $L=L_{1}^{A}$ for some $L_{1}$ in $\mathfrak{I}_{0} \cap \mathfrak{S}_{\text {. }}$. But then $L^{2}=\left(L_{1}^{2}\right)^{A}=Z=Z^{A}$. This is impossible, so $Z_{1} \notin \mathfrak{S}_{\text {. }}$. 
Suppose $Z \in C_{\mathfrak{x}}\left(Z_{1}\right)^{\prime}$. Enlarge $C_{\tilde{\Sigma}}\left(Z_{1}\right)$ to a $S_{2}$-subgroup $\tilde{\mathfrak{I}}=\mathfrak{T}^{X}$ of $\boldsymbol{C}_{\Theta}\left(Z_{1}\right)$. Then $Z \in \tilde{\mathfrak{I}}^{\prime} \leqq \mathfrak{S}^{X}$, so that $Z=Z_{1}$ by the previous paragraph. Hence, $Z \notin C_{\mathfrak{\Sigma}}\left(Z_{1}\right)^{\prime}$. By Lemma 5.36, $\bar{Z}_{1}$ normalizes some $S_{2^{\prime}}$-subgroup of $\overline{\mathfrak{R}}$, so $Z_{1}$ normalizes $\mathfrak{Q}^{H}$ for some $H$ in $\mathfrak{K}$. We may therefore assume that $Z_{1} \in \Re$. Since $\Re / 3$ is cyclic, $\Re$ is abelian. Since $\Re$ contains more than one involution, $\Omega$ is not cyclic. Suppose $|\Re|=8$. Then $Z_{1} \in\langle\boldsymbol{D}(\Re), Z\rangle$, so that $Z_{1}$ centralizes $\mathfrak{M}$. Hence $\Re$ centralizes $\mathfrak{M}$, since $Z \notin C_{\tilde{x}}\left(Z_{1}\right)^{\prime}$. Then by Lemma 5.29, $C_{\mathfrak{5}}\left(Z_{1}\right)=\mathfrak{B}$. Since $\Re$ stabilizes $\left[\mathfrak{S}, Z_{1}\right] \supset \mathfrak{W} \supset 1$, it follows that $\boldsymbol{D}(\Re)$ centralizes $\left[\mathfrak{F}, Z_{1}\right]$. This contradiction shows that $\Re$ is a four-group.

Since $Z_{1} \in \Re$ and $\bar{Z}_{1}$ inverts $\overline{\mathcal{Q}}$, it follows that $C_{\bar{\Re}}\left(\bar{Z}_{1}\right)=\overline{\boldsymbol{C}}_{\mathfrak{\Sigma}}\left(\overline{Z_{1}}\right)$. Thus, $C_{\mathfrak{x}}\left(Z_{1}\right)$ is a $S_{2}$-subgroup of $\boldsymbol{C}_{\Re}\left(Z_{1}\right)$. By Lemma 11.1,

$$
C_{\mathfrak{x}}\left(Z_{1}\right)=\left\langle Z_{1}\right\rangle \times C_{\mathfrak{5}}\left(Z_{1}\right)
$$

is elementary. Hence, $C_{\mathfrak{r}}\left(Z_{1}\right)=\left\langle Z_{1}\right\rangle \times \mathfrak{W}=\mathfrak{F}$, say. Let $\mathfrak{F}=N_{\mathfrak{F}}(\mathfrak{F})$. Thus, $\mathfrak{F} / \mathbb{Z}=N_{\mathfrak{g} / 3}(\mathfrak{F} / \mathbb{Z})$. Since $\mathfrak{W} \triangleleft \mathfrak{I}$, it follows that

$$
\mathfrak{F}=\left\{F \mid F \in \mathfrak{S},\left[F, Z_{1}\right] \in \mathfrak{W}\right\} \text {. }
$$

Since $\bar{Z}_{1}$ inverts $\bar{\complement}$, it follows that

$$
\overline{\mathfrak{S}} / \overline{\mathrm{Z}}=\overline{\mathfrak{S}}_{1} / \overline{\mathrm{Z}} \times \overline{\mathfrak{S}}_{2} / \overline{\mathrm{Z}},
$$

where $\left|\overline{\mathscr{F}}_{i}: \overline{3}\right|=4$ and $\overline{\mathscr{S}}_{i} / \overline{\bar{Z}}$ is an indecomposable $\left\langle\bar{Z}_{1}\right\rangle$-module. This decomposition of $\overline{\mathfrak{F}} / \overline{\widehat{B}}$ shows that $\overline{\mathscr{F}} / \overline{\mathrm{B}}$ is of order 8 . Since $\overline{\mathscr{\mho}} / \overline{\mathrm{B}} \cong$ $\mathfrak{F} / \mathfrak{3},|\mathfrak{F}|=16,\left|\boldsymbol{N}_{\mathfrak{x}}(\mathfrak{F})\right|=32$. This implies that $\mathfrak{W} Z_{1}$ is permuted

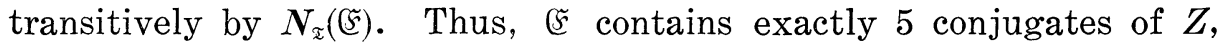
namely, $\{Z\} \cup\left\{\mathfrak{B} Z_{1}\right\}$. Since $N_{\mathfrak{5}}(\mathfrak{F}) / 3$ is elementary, it follows that $N_{\mathfrak{F}}(\mathfrak{S})$ maps onto $A(\mathscr{C})$ where $\mathscr{C}$ is the chain $\mathfrak{F} \supset \mathfrak{W} \supset 1$. Since $2 \epsilon$ $\pi_{2}, N_{\mathfrak{x}}(\mathfrak{F})$ is a $S_{2}$-subgroup of $N_{\mathbb{S}}(\mathfrak{F})$, being of index 2 in $\mathfrak{T}$.

Let $\mathfrak{I}^{*}$ be a $S_{2}$-subgroup of $C_{\mathbb{\Theta}}\left(Z_{1}\right)$ which contains (5. Since $\mathbb{5}$ is generated by conjugates of $Z$, there is a conjugate $E$ of $Z$ which lies

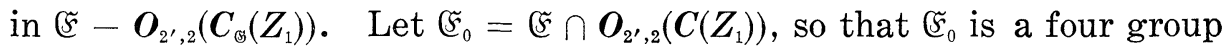
and $\mathfrak{E}_{0} \triangleleft \mathfrak{I}^{*}$. Thus, $\mathfrak{S}_{0} E$ consists of conjugates of $Z$ and $N_{\mathfrak{X}^{*}}(\mathfrak{F})$ maps onto $\boldsymbol{A}\left(\mathscr{C}^{*}\right)$ where $\mathscr{C}^{*}$ is the chain $\left(\mathfrak{F} \supset \mathfrak{F}_{0} \supset 1\right.$.

Let $\mathfrak{B}=\boldsymbol{A}_{\mathscr{G}}(\mathfrak{F})$. Thus, $\boldsymbol{A}_{\widetilde{\Upsilon}}(\mathfrak{F})$ and $\boldsymbol{A}_{\mathscr{\Upsilon}^{*}}(\mathfrak{F})$ are distinct $S_{2}$-subgroups of $\mathfrak{B}$ and are of order 4 . Thus, $\mathfrak{B}$ is a subgroup of Aut (צ्) with the following properties:

(a) $|\mathfrak{B}|_{2}=4$,

(b) $\mathfrak{B}$ is not 2-closed.

As $\sqrt{5}$ is elementary of order 8, it follows by inspection of Aut((5) that no such groups $\mathfrak{B}$ exist.

We may now assume that $\boldsymbol{Z}\left(\Omega_{0}\right)$ is a four-group. Since $2 \in \pi_{2}$, it follows that $\Omega_{0} / 3\left(\Omega_{0}\right)$ is of order $2^{4}$ and $p=5$. Furthermore, $\&=\Omega_{0}$, for the same reason. By Lemma 5.26, it follows that $\mathfrak{M}_{5} \mathcal{Q} / \boldsymbol{O}_{5}(\mathfrak{M})$ is 
isomorphic to the centralizer of an involution of $U_{3}(4)$.

We will show that 3 is weakly closed in $\mathfrak{T}$. First suppose $|\mathfrak{Z}|=$ 4 , so that $3=Z\left(\Omega_{0}\right)$. In this case, any 2 -subgroup of (8) which contains 3 and centralizes some element of $3^{*}$ centralizes 3 , since for each $X$ in $3^{\sharp}, 3 \subseteq Z\left(C(X) \bmod \boldsymbol{O}_{2^{\prime}}(\boldsymbol{C}(X))\right)$. If $3^{G} \subset \mathfrak{I}$, then since $\mathfrak{I} / \mathbb{R}$ is cyclic, we have $3^{G} \cap \mathbb{2} \neq 1$, so $3^{G} \cap 3 \neq 1$, so $3=3^{G}$. We may assume that $|\mathfrak{Z}|=2$. Hence $\mathbb{C} \subset \mathfrak{I}$. In this case, we first verify that $I \Varangle Z$. Suppose false. Then $\mathfrak{W}=\boldsymbol{Z}(\mathbb{R})$ and $\boldsymbol{A}_{\mathfrak{G}}(\mathfrak{W})=\operatorname{Aut}(\mathfrak{W})$. Let $\mathfrak{R} *=\boldsymbol{N}_{\mathfrak{G}}(\mathfrak{W})$. Then a $S_{2^{\prime}}$-subgroup $\mathfrak{\Omega}^{*}$ of $\mathfrak{R}^{*} / \boldsymbol{O}_{2^{\prime}}\left(\mathfrak{R}^{*}\right)$ is of order 15 and a generator $Q^{*}$ of $\mathfrak{\Omega}$ is inverted by some element of $\mathfrak{R}^{*} / \boldsymbol{O}_{2^{\prime}}\left(\mathfrak{N}^{*}\right)$. Since $\boldsymbol{O}_{2^{\prime}, 2}\left(\mathfrak{N}^{*}\right)=$ $2 O_{2^{\prime}}\left(\Re^{*}\right)$, it follows that the characteristic roots of $Q^{*}$ on $\boldsymbol{O}_{2^{\prime}, 2}\left(\Re^{*}\right) / \mathfrak{M O}_{2^{\prime}}\left(\mathfrak{R}^{*}\right)$ are $\lambda, \lambda^{2}, \lambda^{4}, \lambda^{8}$ for some primitive 15 th root of 1 in $F_{16}$. Thus, $Q^{*}$ is non real in $\mathfrak{R}^{*} / \boldsymbol{O}_{2^{\prime}}\left(\mathfrak{R}^{*}\right)$. This contradiction shows that $I \nsim Z$.

Suppose $Z^{G}=Z_{1} \in \mathfrak{I}, Z_{1} \neq Z$. By the preceding argument, $Z_{1} \in \mathfrak{I}$. Let $\tilde{\mathfrak{T}}=\mathfrak{T}^{x}$ be a $S_{2}$-subgroup of $C\left(Z_{1}\right)$ which contains $\boldsymbol{C}_{\mathfrak{\Sigma}}\left(Z_{1}\right)$. If $Z \in$ $\boldsymbol{C}_{\mathfrak{\Sigma}}\left(Z_{1}\right)^{\prime}$, then $Z \in \mathbb{Q}^{x}$, so that $Z=Z_{1}$. Hence $Z \notin \boldsymbol{C}_{\mathfrak{x}}\left(Z_{1}\right)^{\prime}$.

Suppose $Z_{1}$ centralizes $\mathfrak{W}$. Then $\mathfrak{W} \cap \mathfrak{Q}^{X} \neq 1$, so that $\mathfrak{W} \cap \mathfrak{S}^{x}=$ $\langle I\rangle$. In this case, $C_{\tilde{x}}(I)$ and $C_{\tilde{x}}(I)$ are both $S_{2}$-subgroups of $C(I)$, so $Z_{1} \in \boldsymbol{Z}\left(\boldsymbol{O}_{2^{\prime}, 2}\left(\boldsymbol{C}(I) \bmod \boldsymbol{O}_{2^{\prime}}(\boldsymbol{C}(I))\right)\right.$. This is not the case, since $\left[Z_{1}, \mathrm{Q}\right]$ is not elementary. Hence $Z_{1}$ does not centralize $\mathfrak{W}$. Hence, $\mathfrak{T} / \mathbb{R}$ is of order 2. Since $\left|C_{\mathcal{R}}\left(Z_{1}\right)\right| \geqq 4$, it follows that $Z \in \boldsymbol{D}\left(\boldsymbol{C}_{\mathbb{8}}\left(Z_{1}\right)\right)$, so that $Z \in \mathfrak{Q}^{X}$. This is not the case, so 3 is weakly closed in $\mathfrak{I}$.

By (11.1), we conclude that $|3|=4$. By a standard transfer theorem, $\mathfrak{I} \subseteq N(\mathfrak{I})^{\prime}$. Hence $\mathbb{Z}=\mathfrak{I}$. By (11.1) once again, the three involutions of 3 are fused in $N(\mathfrak{T})$. The lemma follows.

Lemma 11.7. Suppose $|3|=2, \mid \mathscr{C}(\mathfrak{I})=1$ and $I \underset{\leftrightarrow}{\not} Z$. Then one of the following holds:

(a) ( i ) $\boldsymbol{C}_{\Theta}(I)=\boldsymbol{O}_{2^{\prime}}\left(\boldsymbol{C}_{\mathfrak{\leftrightarrow}}(I)\right) \cdot \boldsymbol{C}_{\circlearrowleft}(\mathfrak{B})$.

(ii) If $\mathfrak{B}$ is any 2-subgroup of $\mathbb{C}(I)$ which contains $\mathfrak{W}$, then $\mathfrak{B}$ centralizes $\mathfrak{B}$.

(b) $|\mathfrak{I}|=2^{5}$.

Proof. Since (a)(ii) is a consequence of (a)(i), it suffices to show that either (a)(i) or (b) holds.

Set $\mathbb{C}=C(I), \mathfrak{I}_{0}=C_{\mathfrak{x}}(I)$. Since $I \nsim Z$, it follows that $\mathfrak{I}_{0}$ is a

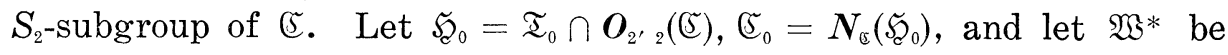
the normal closure of $\mathfrak{W}$ in $\mathfrak{E}_{0}$. Notice that since $\mathfrak{W} \subseteq \boldsymbol{Z}\left(\mathfrak{I}_{0}\right)$, Lemma 5.10 implies that $\mathfrak{W}^{*}$ is 2 -reducible in $\mathfrak{C}_{0}$. In particular, $\mathfrak{W}^{*} \subseteq \boldsymbol{Z}\left(\mathfrak{S}_{0}\right)$.

By Lemma 11.6, $\boldsymbol{A}_{\mathbb{E}_{0}}\left(\mathfrak{W}^{*}\right)$ is a 2, 3-group. If $\mathfrak{W}^{*}=\mathfrak{W}$, it follows that $\mathfrak{W}^{*} \subseteq \boldsymbol{Z}\left(\mathbb{S}_{0}\right)$, so that (a)(i) holds, since $\mathbb{C}=\boldsymbol{O}_{2^{\prime}}(\mathfrak{\complement}) \cdot \mathfrak{S}_{0}$. Hence, we 
may assume that $\mathfrak{W} \supset \mathfrak{W}$.

Since $\mathscr{S C C N}_{3}(\mathfrak{T})=\varnothing$, it follows that $\mathscr{S} \mathscr{C} \mathscr{N}_{6}\left(\mathfrak{I}_{0}\right)=\varnothing$. Namely, let $\mathbb{F}$ be a normal elementary subgroup of $\mathfrak{I}_{0}$ of maximal order. Thus, $\mathfrak{W} \subseteq \mathbb{F}$, as $\mathfrak{W} \subseteq Z\left(\mathfrak{I}_{0}\right)$. Choose $T$ in $\mathfrak{I}-\mathfrak{I}_{0}$. Then $\mathbb{F} \cap \mathfrak{F}^{T}$ is a normal elementary subgroup of $\mathfrak{T}$, so $\mathfrak{F} \cap \mathfrak{F}^{T}=\mathfrak{W}$. Thus, $\left\langle\mathfrak{F}, \mathfrak{F}^{T}\right\rangle$ is a normal subgroup of $\mathfrak{I}$ of exponent $\leqq 4$ and of order $2^{2(1+e)}$ where $|\mathfrak{F}|=2^{2+e}$. Let $\mathfrak{A} \in \mathscr{S} \mathscr{C} \mathscr{N}\left(\left\langle\mathfrak{G}, \mathfrak{F}^{T}\right\rangle\right), \mathfrak{U} \backslash \mathfrak{I}$. Thus, $\mathfrak{N}$ is of type $\left(2^{a_{1}}, 2^{a_{2}}\right)$, where $1 \leqq a_{1} \leqq a_{2} \leqq 2$, and $\Omega_{1}(\mathfrak{U})=\mathfrak{W}$. Since $\left\langle\mathfrak{F}\right.$, $\left.\mathfrak{F}^{T}\right\rangle$ centralizes $\mathfrak{W}$, $\left\langle\mathfrak{G}\right.$, $\left.\mathfrak{F}^{T}\right\rangle$ stabilizes $\mathfrak{A} \supseteqq \mathfrak{W} \supseteqq 1$. The stability group of this chain has order at most $2^{4}$, so that $\mid\left\langle\mathfrak{F}\right.$, $\left.\mathfrak{F}^{T}\right\rangle: \mathfrak{A} \mid \leqq 2^{4}$. Since $|\mathfrak{A}| \leqq 2^{4}$, we get $\left|\left\langle\mathfrak{F}, \mathfrak{F}^{T}\right\rangle\right| \leqq 2^{8}$, so that $e \leqq 3$, as asserted. Hence $\left|\mathfrak{S}^{*}\right|=2^{n}$, where $3 \leqq n \leqq 5$. It follows that $\boldsymbol{A}_{\mathfrak{\mho}_{0}}\left(\mathfrak{W}^{*}\right)$ does not contain an elementary group of order $3^{3}$. By Lemma 5.34, $\boldsymbol{A}_{\mathfrak{\Xi}_{0}}\left(\mathfrak{W}^{*}\right)$ does not contain an elementary subgroup of order $2^{3}$. Choose $T \in \mathfrak{I}-\mathfrak{I}_{0}$. Then

$$
\mathfrak{W}^{*} \cap \mathfrak{W}^{* T}=\mathfrak{W}=\Omega_{1}(\boldsymbol{Z}(\langle\mathfrak{W} *, \mathfrak{W} * T\rangle)) \text {. }
$$

Since $\mathfrak{W}^{* T} \cap \boldsymbol{C}_{\mathbb{E}_{0}}\left(\mathfrak{W}^{*}\right)$ is in the center of $\left\langle\mathfrak{W}^{*}, \mathfrak{W}^{* T}\right\rangle$, it follows that $\mathfrak{W}^{* T} \cap \boldsymbol{C}\left(\mathfrak{W}^{*}\right)=\mathfrak{W}$. Hence $\mathfrak{W}^{* T} / \mathfrak{W}^{* T} \cap \boldsymbol{C}\left(\mathfrak{W}^{*}\right)$ is elementary of order $2^{n-2}$, so $n \leqq 4$. Suppose $n=4$. Then

$$
\mathfrak{W}^{* T} / \mathfrak{W}^{* T} \cap \boldsymbol{C}\left(\mathfrak{W}^{*}\right) \cong \mathfrak{W}^{* T} \boldsymbol{C}_{\mathfrak{E}_{0}}\left(\mathfrak{W}^{*}\right) / \boldsymbol{C}_{\mathbb{E}_{0}}\left(\mathfrak{W}^{*}\right)
$$

is a fourgroup. By Lemma 5.34, $\boldsymbol{O}_{2^{\prime}}\left(\boldsymbol{A}_{\varpi_{0}}\left(\mathfrak{W}^{*}\right)\right)$ contains a subgroup which is the direct product of 2 groups of prime order. This is impossible since $\left|\mathfrak{W}^{*}\right|=2^{4}$ and $I \in \boldsymbol{Z}\left(\mathfrak{C}_{0}\right)$. Hence, $n \leqq 3$, so that $n=3$.

Since $n=3$, it follows that $\mathfrak{F}^{*}=\langle I\rangle \times \mathfrak{A}$, where $\mathfrak{A} \triangleleft \mathfrak{S}_{0}$ and $\boldsymbol{A}_{\mathbb{E}_{0}}(\mathfrak{R})=\operatorname{Aut}(\mathfrak{X})$. Let $\mathfrak{I}_{1}=\boldsymbol{C}_{\mathfrak{x}_{0}}\left(\mathfrak{W}^{*}\right), \mathfrak{C}_{1}=\boldsymbol{N}_{\mathbb{E}_{0}}\left(\mathfrak{I}_{1}\right)$, so that $\left|\mathfrak{I}_{0}: \mathfrak{I}_{1}\right|=2$ and $\mathfrak{S}_{1}$ maps onto $\boldsymbol{A}_{\mathbb{\varpi}_{0}}\left(\mathfrak{W}^{*}\right)$. Choose $T$ in $\mathfrak{I}-\mathfrak{I}_{0}$, and then choose $X$ in $\mathfrak{S}^{* T}-\mathfrak{I}_{1}$. We can then choose $R$ of odd order in $\mathfrak{S}_{1}$ such that $R$ is inverted by $X$ and does not centralize $\mathfrak{S}^{*}$, by Lemma 5.36. Since $\left[T_{0}, X\right]$ is of order 2 , it follows that $\mathfrak{I}_{1}=\mathfrak{A} \times \mathfrak{B}$, where $\mathfrak{B}=C_{\mathfrak{x}_{1}}(R)$. Hence, $\mathfrak{B}$ is centralized by $X$ and $I \in \mathfrak{B}$. If $Z \in \mathfrak{B}$, then $\mathfrak{B} \subseteq \mathfrak{B}$, so that $\mathfrak{W} * \supseteqq \mathfrak{A} \mathfrak{W}=\mathfrak{A} \times \mathfrak{W}$, a group of order 16. This is not the case, so $Z \notin \mathfrak{B}$. Suppose $\mathfrak{B}^{\prime} \neq 1$. Since $\left|\mathfrak{I}_{0}: \mathfrak{I}_{1}\right|=2$, it follows that $\mathfrak{I}_{1} \cap \mathfrak{I}_{1}^{T}$ is of index 4 in $\mathfrak{I}_{0}$, so of index 2 in $\mathfrak{I}_{1}$. Since $\mathfrak{I}_{1} \cap \mathfrak{I}_{1}^{T}$ centralizes $\mathfrak{W}^{* T}$, it follows that $\mathfrak{W}^{*} \nsubseteq \mathfrak{I}_{1} \cap \mathfrak{I}_{1}^{T}$. Hence $\mathfrak{I}_{1}=\mathfrak{S}^{*}\left(\mathfrak{I}_{1} \cap \mathfrak{I}_{1}^{T}\right)$. Since $\mathfrak{W}^{*} \subseteq \boldsymbol{Z}\left(\mathfrak{I}_{1}\right)$, and since $\mathfrak{I}_{1}$ is assumed nonabelian, we get

$$
\mathfrak{I}_{1}^{\prime}=\left(\mathfrak{I}_{1} \cap \mathfrak{I}_{1}^{T}\right)^{\prime} \triangleleft \mathfrak{I} \text {. }
$$

Hence, $Z \in \mathfrak{T}_{1}^{\prime}=\mathfrak{B}^{\prime}$, against $Z \notin \mathfrak{B}$. Hence, $\mathfrak{B}$ is abelian.

Since $\mathfrak{B} \subseteq Z\left(\mathfrak{I}_{0}\right)$, it follows that $\sigma^{1}\left(Z\left(\mathfrak{I}_{0}\right)\right)=\sigma^{1}(\mathfrak{B}) \triangleleft \mathfrak{I}$. Since $Z \notin$ $\mathfrak{B}, \mathfrak{B}$ is elementary. Since $\Omega_{1}\left(\boldsymbol{Z}\left(\mathfrak{I}_{0}\right)\right)=\mathfrak{W}$, it follows that $\mathfrak{B}=\langle I\rangle$. Hence $|\mathfrak{I}|=2\left|\mathfrak{I}_{0}\right|=2^{2}\left|\mathfrak{I}_{1}\right|=2^{3}|\mathfrak{A}|=2^{5}$, so that (b) holds. 
The following lemma is the most elaborate of this section.

Lemma 11.8. Suppose $|B|=2,|\mathscr{Q}(\mathfrak{T})|=1$ and $\overline{\mathfrak{S}} \pitchfork \overline{\mathfrak{M}}$. Then $\mathfrak{I}$ is isomorphic to $Z_{4}>Z_{2}$.

Proof. Since $\mathfrak{W} \in \mathscr{K}(\mathfrak{T})$, Lemma 6.1 implies that $\mathfrak{W} \subseteq \mathfrak{F}$. Since $\overline{\mathfrak{W}} \pitchfork \overline{\mathfrak{R}}, \mathfrak{W} \subset \mathfrak{S}$.

If $\mathfrak{S}$ contains a noncyclic characteristic abelian subgroup $\mathbb{R}$, then $\overline{\mathfrak{B}}=\Omega_{1}(\overline{\mathfrak{Q}}) \triangleleft \overline{\mathfrak{R}}$, contrary to hypothesis. Thus, $\mathfrak{F}$ is of symplectic type. Since $\mathfrak{M} \subset \mathfrak{F}$, the width of $\mathfrak{F}$ is at least 1 . Suppose by way of contradiction that the width of $\mathfrak{S}$ is $\geqq 2$. By Lemma 11.3(a), we get that $\mathfrak{F}$ is extra special of order $2^{5}$. By Lemma 11.2, it follows that $\mathfrak{S} \subset \mathfrak{I}$. Suppose $|\mathfrak{T}: \mathfrak{F}|=2$.

We will show that if $J \in \mathfrak{F}, J \neq Z$, then $J \underset{\in}{\ngtr} Z$. Suppose false. Let $\mathfrak{F}_{0}$ be a $S_{2}$-subgroup of $\mathfrak{R} \cap C(J)$ and choose $N$ in $\mathfrak{R}$ such that $\mathfrak{F}_{0}^{N}=\mathfrak{F} \leqq \mathfrak{I}$. Let $I=J^{N}$, so that $I \neq Z, I \widetilde{\mathscr{\sigma}} Z$. Thus $\mathfrak{F} \subset \mathfrak{I}$ and $\mathbb{F} \cap \mathfrak{S}$ is of index 2 in $\mathfrak{S}$. If $\mathbb{F}=\mathbb{F} \cap \mathfrak{F}$, then Lemma 11.1 is violated. Thus $|\mathfrak{F}|=2^{5}$, $|\mathfrak{F} \cap \mathfrak{S}|=2^{4}$. Let $\mathfrak{I}^{*}$ be a $S_{2}$-subgroup of $C(I)$ which contains $\mathfrak{F}$. Thus $\left(\mathfrak{F}=\mathfrak{I} \cap \mathfrak{I}^{*} \triangleleft\left\langle\mathfrak{T}, \mathfrak{I}^{*}\right\rangle=\mathfrak{L}\right.$, say. Since $|\mathscr{Q}(\mathfrak{I})|^{\prime}=1$, we get $\mathfrak{W}=\langle I, Z\rangle=\Omega_{1}(\boldsymbol{Z}(\mathfrak{E}))$ and so $\mathbb{L}$ maps onto Aut $(\mathfrak{W})=\boldsymbol{A}_{\mathscr{G}}(\mathfrak{W})$. Now $\mathscr{S} \cap \mathbb{E} / \mathfrak{W}$ is a four-group, so that $\mathfrak{F} / \mathfrak{W}$ is of order 8 and contains a four-group. Let $R$ be a 3 -element of $N(\mathfrak{S})$ which permutes $I, Z, I Z$ transitively. Let $\mathfrak{U} / \mathfrak{W}=C_{\mathbb{E} / \mathfrak{W}}(R)$. We argue that $|\mathfrak{U}: \mathfrak{W}|=2$. As $|\mathfrak{F}: \mathfrak{W}|=8$, we certainly have $|\mathfrak{U}: \mathfrak{W}| \geqq 2$. If $|\mathfrak{U}: \mathfrak{W}|>2$, then $R$ centralizes $\mathfrak{F} / \mathfrak{W}$ so that $\mathfrak{F}=\mathfrak{W} \times \boldsymbol{C}_{\mathfrak{E}}(\mathfrak{R})$, against $\mathfrak{W}=\Omega_{1}(\boldsymbol{Z}(\mathfrak{F}))$. Hence, $|\mathfrak{U}: \mathfrak{W}|=2$.

Since $|\mathfrak{U}: \mathfrak{W}|=2$ and since $\mathfrak{F} / \mathfrak{W}$ is not a quaternion group, it follows that $\mathfrak{F} / \mathfrak{W}$ is elementary. Since $\boldsymbol{A}_{\mathcal{8}}(\mathfrak{F} / \mathfrak{W}) \cong \Sigma_{3}$, it follows that $\mathfrak{u} \triangleleft \mathfrak{R}$. In particular, $\mathfrak{U} \triangleleft \mathfrak{T}$. But $R$ induces an automorphism of $\mathfrak{U}$ of order 3 and $|\mathfrak{U}|=8$. Since $\mathfrak{W}=[\mathfrak{M}, R]$, it follows that $\mathfrak{U}$ is elementary of order 8 , against $2 \in \pi_{2}$. We conclude that no element of $\mathfrak{S}-3$ is conjugate to $Z$.

Choose $G$ in (8) such that $Z_{1}=Z^{G} \in \mathfrak{T}, Z_{1} \neq Z$. By the previous argument, $Z_{1} \notin \mathfrak{S}_{\mathcal{E}}$. Since $\overline{C_{\mathfrak{z}}\left(Z_{1}\right)}=C_{\overline{\bar{x}}}\left(\bar{Z}_{1}\right)$, and since $\mathfrak{I}=\mathfrak{S}_{\mathfrak{c}}\left\langle Z_{1}\right\rangle$, it follows that $C_{\Sigma}\left(Z_{1}\right)$ is a $S_{2}$-subgroup of $C_{\mathfrak{R}}\left(Z_{1}\right)$. By Lemma 11.1, we get that $C_{\tilde{x}}\left(Z_{1}\right)$ is elementary. By uniqueness of $\mathfrak{W}$, it follows that

$$
C_{\mathfrak{p}}\left(Z_{1}\right)=\mathfrak{W},
$$

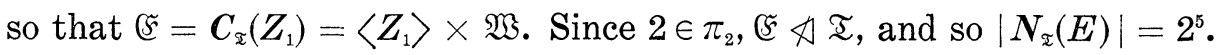
By symmetry, $\left|N_{\mathbb{\varkappa}^{*}}(\mathfrak{\xi})\right|=2^{5}$, where $\mathfrak{I}^{*}$ is a $S_{2}$-subgroup of $C\left(Z_{1}\right)$ which contains $\mathbb{5}$. Thus $\boldsymbol{A}_{\mathscr{\Theta}}(\mathbb{E})$ is not 2-closed and has $S_{2}$-subgroups of order 4. We have seen that Aut(ङ) has no such subgroups. We conclude that $|\mathfrak{T}: \mathfrak{S}| \geqq 4$. 
By Lemma 11.5, we get that $\overline{\mathfrak{D}}$ is a 3-group. Since $\overline{\mathfrak{I}} / \overline{\mathfrak{F}}$ is faithfully represented on $\boldsymbol{O}_{2,2^{\prime}}(\overline{\mathfrak{M}}) / \overline{\mathfrak{F}}$ and since $\overline{\mathfrak{F}}$ is extra special of order $2^{5}$, we conclude that $\overline{\mathfrak{D}} \overline{\mathfrak{S}}=\boldsymbol{O}_{2,2^{\prime}}(\overline{\mathfrak{\Re}})$ and that $\overline{\mathfrak{D}}$ is elementary of order $3^{2}$. This implies that $\mathfrak{S}$ is the central product of 2 quaternion groups $\mathfrak{F}_{1}, \mathfrak{S}_{2}$. Furthermore, as $\mathfrak{F}_{1}, \mathfrak{F}_{2}$ are the only quaternion subgroups of $\mathfrak{K}$, it follows that $N_{\tilde{乏}}\left(\mathscr{S}_{1}\right)=N_{\tilde{\varepsilon}}\left(\mathscr{S}_{2}\right)$ is of index at most 2 in T. Also, $N_{\mathfrak{x}}\left(\mathfrak{S}_{\mathcal{1}}\right) / \mathfrak{F}$ is elementary of order $\leqq 4$, and so $\left|\mathfrak{T}: \mathfrak{F}_{\mathfrak{C}}\right| \leqq 8$. As $\overline{\mathfrak{I}} / \overline{\mathscr{S}}$ is faithfully represented on $\overline{\mathfrak{D}}$, and as $S_{2}$-subgroups of Aut (馬) are semi-dihedral of order 16 , it follows that $\mathfrak{I} / \mathfrak{S}$ is one of the following:

(a) cyclic of order 4.

(b) a four-group.

(c) a dihedral group of order 8 .

Let $\Re_{i}=C_{\mathfrak{Q}}\left(\overline{\mathscr{S}}_{i}\right), i=1,2$, so that $\left|\bar{\Re}_{i}\right|=3, i=1,2$. Also

$$
\mathfrak{R}_{i}=\left\langle R_{i}, \boldsymbol{O}_{2^{\prime}}(\mathfrak{R})\right\rangle
$$

for suitable 3-elements $R_{i}$. Let

$$
\Re_{3}=\left\langle R_{1} R_{2}, O_{2^{\prime}}(\mathfrak{R})\right\rangle \quad \Re_{4}=\left\langle R_{1} R_{2}^{-1}, O_{2^{\prime}}(\mathfrak{N})\right\rangle .
$$

Thus, $\bar{\Re}_{i}$ exhaust the subgroups of $\overline{\mathfrak{D}}$ of order $3,1 \leqq i \leqq 4$. Also $\boldsymbol{C}_{\overline{\bar{b}}}\left(\bar{\Re}_{i}\right)=\overline{3}, i=3,4$.

We next show that $\mathfrak{I}-\mathfrak{F}$ contains a conjugate of $Z$. Suppose false. Choose $Z_{1}=Z^{G} \in \mathfrak{T}, Z \neq Z_{1}$, such that $C_{\check{z}}\left(Z_{1}\right)$ is a $S_{2}$-subgroup of $C_{\Re}\left(Z_{1}\right)$. Then $Z_{1} \in \mathfrak{S}_{\text {. }}$. Since $|\overline{\mathfrak{D}}|=3^{2}$, it follows that $\overline{\mathfrak{F}} \cong$ permutes transitively the 18 noncentral involutions of $\overline{\mathcal{F}}$. Thus, every involution of $\mathfrak{F}$ is conjugate to $Z$. Since $C_{\mathfrak{5}}\left(Z_{1}\right)$ is the direct product of $\left\langle Z_{1}\right\rangle$ and a dihedral group of order $8, C_{\mathfrak{5}}\left(Z_{1}\right)$ is generated by conjugates of $Z$, so $C_{\mathfrak{5}}\left(Z_{1}\right)$ is weakly closed in $C_{\mathfrak{x}}\left(Z_{1}\right)$. Thus, $\langle Z\rangle$ char $C_{\mathfrak{5}}\left(Z_{1}\right)$, against Lemma 11.1. Hence, $\mathfrak{I}-\mathfrak{S}$ contains conjugates of $Z$.

Let $J$ be an involution of $\mathfrak{I}-\mathfrak{S}$. We need to relate $C_{\mathfrak{g}}(J)$ to the set of fixed points of $\bar{J}$ on $\overline{\mathfrak{D}} \overline{\mathcal{F}} / \overline{\mathscr{S}}$. First suppose that $\bar{J}$ inverts

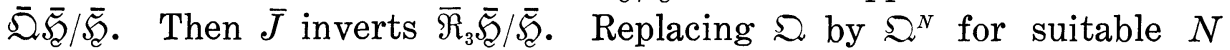
in $\mathfrak{N}$ and applying Lemma 5.36, we may assume that $\bar{J}$ inverts $\bar{\Re}_{3}$.

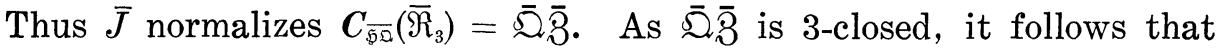
with our normalization, we get that $\bar{J}$ inverts $\bar{\beth}$. Hence, $\bar{J}$ normalizes $\overline{\mathfrak{F}}_{i}=C_{\overline{\mathfrak{F}}}\left(\Re_{i}\right), i=1,2$. Thus, $\left\langle\bar{J}, \bar{\Re}_{2}\right\rangle \cong \Sigma_{3}$ and $\left\langle\bar{J}, \bar{\Re}_{2}\right\rangle$ is faithfully represented on $\mathfrak{S}_{1}$. Similarly, $\left\langle\bar{\Im}, \bar{\Re}_{1}\right\rangle$ is faithfully represented on ${\overline{\mathfrak{F}_{2}}}_{2}$. We can therefore choose generators $H_{i 1}, H_{i 2}$ of $\mathscr{S}_{i}$ such that $H_{i 1}^{J}=H_{i 2}, i=1,2$. Thus, $J$ inverts $H_{i 1} H_{12}$ and

$$
\boldsymbol{C}_{\mathfrak{⿱}_{i}}(J)=\langle Z\rangle \text {. }
$$

This implies that $C_{\mathfrak{g}}(J)=\left\langle Z, H_{11} H_{12} H_{21} H_{22}\right\rangle$, a four-group. Suppose

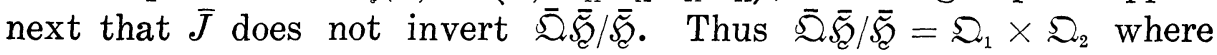


$\mathfrak{Q}_{1}$ is centralized by $\bar{J}$ and $\mathfrak{\Omega}_{2}$ is inverted by $\bar{J}$. Thus $\mathfrak{\Omega}_{2}=\bar{\Re}_{i} \overline{\mathfrak{S}} / \overline{\mathfrak{C}}$ for a uniquely determined $i \in\{1,2,3,4\}$. Replacing $\mathfrak{Q}$ by a suitable $\mathfrak{\Omega}^{N}, N \in \mathfrak{R}$, and using Lemma 5.36, we may assume that $\bar{J}$ inverts $\bar{\Re}_{i}$. If $i \in\{1,2\}$, then $\bar{J}$ normalizes $\overline{\mathfrak{S}}_{i}=C_{\mathfrak{F}}\left(\bar{\Re}_{i}\right)$, so that $\bar{J}$ normalizes both ${\overline{\mathcal{F}_{1}}}_{1}$ and $\overline{\mathscr{S}}_{2}$. Choose $j \in\{1,2\}, j \neq i$. Then $\langle\bar{J}, \overline{\mathfrak{D}} \overline{\mathcal{S}}\rangle$ is represented on $\overline{\mathfrak{F}}_{i}$, and since $\bar{J}$ centralizes $\mathfrak{\Omega}_{1}$, it follows that $\bar{J}$ induces an inner automorphism of $\overline{\mathfrak{F}}_{i}$. In particular, $C_{\mathfrak{5}}(J)$ is not elementary. Suppose $i \in\{3,4\}$. In this case, $\bar{J}$ normalizes $\boldsymbol{C}_{\mathfrak{p} 0}\left(\bar{\Re}_{i}\right)=\overline{3} \overline{\mathfrak{O}}$, so $\bar{J}$ normalizes ㄱ. Choose $j \in\{3,4\}, j \neq i$. Since $\bar{\Re}_{i}$ and $\bar{\Re}_{j}$ are the only subgroups of $\bar{Q}$ of order 3 which do not centralize quaternion subgroups of $\overline{\mathcal{F}}$, it follows that $\bar{J}$ normalizes $\bar{\Re}_{j}$, so $\bar{J}$ centralizes $\bar{\Re}_{j}$. This then implies that $\bar{\Re}_{1}^{J}=\bar{\Re}_{2}$. Hence $\mathfrak{S}_{1}^{J}=\mathfrak{S}_{2}$ and $C_{\mathfrak{5}}(J)$ is elementary of order 8. We may thus draw up a table exhibiting the relationships just discovered.

\begin{tabular}{|c|c|c|}
\hline $\mathrm{C}_{\overline{\mathfrak{s}} \bar{\Omega} / \overline{\overline{5}}}(\bar{J})$ & $\mathbb{C}_{\mathfrak{5}}(J)$ & $\mathfrak{H}_{1}^{J}$ \\
\hline 1 & a four-group & $\mathfrak{F}_{1}$ \\
\hline$\overline{\Re_{i}} ; \overline{\mathfrak{S}} / \overline{\mathfrak{S}}, i \in\{1,2\}$ & not elementary & $\mathfrak{H}_{1}$ \\
\hline $\bar{\Re}_{i} \overline{\bar{S}} / \sqrt{\mathfrak{S}}, i \in\{3,4\}$ & elementary of order 8 & $\mathfrak{S}_{2}$ \\
\hline
\end{tabular}

Here $J$ is any involution of $\mathfrak{I}-\mathfrak{S}$. We remark that if $\bar{J}$ is a square in $\overline{\mathfrak{N}} / \overline{\mathfrak{S}}$, then the first possibility holds.

For the remainder of this lemma, $Z_{1}$ denotes a conjugate of $Z$ such that

$$
\begin{aligned}
& \text { (i) } Z_{1} \in \mathfrak{I}-\mathfrak{S} \\
& \text { (ii) } \mathfrak{F}=C_{\mathfrak{\Sigma}}\left(Z_{1}\right) \text { is a } S_{2} \text {-subgroup of } C_{\Re}\left(Z_{1}\right) \text {. }
\end{aligned}
$$

As we have seen, $Z_{1}$ is available satisfying (i). If (ii) is not satisfied for a given $Z_{1}$ which satisfies (i), let $\xi_{0}$ be a $S_{2}$-subgroup of $C_{\Re}\left(Z_{1}\right)$ and choose $N$ in $\mathfrak{N}$ such that $\mathbb{F}=\mathfrak{S}_{0}^{N} \subseteq \mathfrak{I}$. Then $Z_{1}^{\prime}=Z_{1}^{N}$ satisfies (ii). If $Z_{1}^{\prime} \in \mathfrak{S}$, we get $\bar{Z}_{1} \notin \overline{\mathscr{F}}, \bar{Z}_{1}^{\prime} \in \overline{\mathscr{F}}$ against $\overline{\mathfrak{F}} \triangleleft \overline{\mathfrak{R}}$. So the conclusion is that $Z_{1}$ is always available satisfying (i) and (ii).

We now normalize a little more. Namely, we choose a $S_{2^{\prime}}$-subgroup $\mathfrak{Q}$ of $\mathfrak{R}$ such that

(iii) $\bar{Z}_{1}$ inverts some subgroup of $\overline{\mathcal{D}}$ of order 3.

(iv) If $\bar{Z}_{1}$ inverts $\overline{\mathfrak{D}} \overline{\mathfrak{F}} / \overline{\mathcal{S}}$, then $Z_{1}$ inverts $\overline{\mathfrak{D}}$.

So if (iv) holds, $Z_{1} \in \Re$. Notice also that if $\bar{Z}_{1}$ inverts $\bar{\Re}_{i}$ with $i \epsilon$ $\{3,4\}$, then $\bar{Z}_{1}$ normalizes $\overline{\mathfrak{Q}}=\boldsymbol{O}_{3}\left(C_{\overline{\mathfrak{s}} \bar{\Omega}}\left(\bar{\Re}_{i}\right)\right)$.

Case (a). $\mathfrak{I} / \mathfrak{H}$ is cyclic of order 4.

Here $\bar{Z}_{1}$ is a square in $\overline{\mathfrak{N}} / \overline{\mathfrak{F}}$, so the first possibility in (11.2) holds. Hence, $\widetilde{F} \cap \mathfrak{F}$ is a four-group. Since $\Re / 3$ is cyclic, $\mathscr{\Re}$ is abelian, so $\Re$ normalizes $\mathfrak{F} \cap \mathfrak{S}$. Hence $\mathfrak{F} \cap \mathfrak{K}=\mathfrak{W}$, by uniqueness of $\mathfrak{W}$. Also $\mathfrak{F}=\mathscr{R}(\mathfrak{F} \cap \mathfrak{S})$ is of order 16 . Let $\mathfrak{U}$ be a subgroup of $\mathfrak{S}$ of order 8 
which contains $\mathfrak{W}$ and is normal in $\mathfrak{T}$. If $\Re$ centralizes $\mathfrak{W}$, then $\Re$ stabilizes $\mathfrak{U} \supset \mathfrak{W} \supset 1$. As $\mathfrak{W}$ is elementary, $\boldsymbol{D}(\mathfrak{R})$ centralizes $\mathfrak{H}$. Since $Z_{1} \in\langle\boldsymbol{D}(\Re), Z\rangle, Z_{1}$ centralizes $\mathfrak{H}$. This is not the case, so [尺, $\left.\mathfrak{W}\right]=3$. Since $\mathfrak{S}=\Re \mathfrak{R}$, it follows that $\mathfrak{G}^{\prime}=3$, against Lemma 11.1 .

Case (b)(i). $\mathfrak{I} / \mathfrak{S}$ is a four-group and $C_{\mathfrak{F}}\left(Z_{1}\right)$ is not elementary.

By (11.2), $Z_{1}$ normalizes both $\mathscr{S}_{1}$ and $\mathfrak{K}_{2}$. As $C_{\mathfrak{y}}\left(Z_{1}\right)$ is not a four-group, $Z_{1}$ induces an outer automorphism of exactly one of $\mathfrak{K}_{1}$, $\mathfrak{S}_{2}$, say $\mathfrak{K}_{2}$. Thus, $\mathfrak{S}_{2}$ has generators $H_{21}, H_{22}$ with $H_{21}^{Z_{1}}=H_{22}$, so that $Z_{1}$ inverts $H_{21} H_{22}$. If $\mathbb{F}=\left\langle Z_{1}\right\rangle \times \mathbb{F} \cap \mathfrak{K}_{\mathrm{K}}$, then 3 char $₹$, against Lemma 11.1. Thus we may assume that $\mid \mathfrak{F}$ : $\mathbb{E} \cap \mathfrak{F} \mid=4$, so that $\mathbb{E}$ covers $\mathfrak{I} / \mathfrak{S}_{2}$. First, suppose that $Z_{1}$ centralizes $\mathfrak{S}_{1}$, so that $C_{\mathfrak{F}}\left(Z_{1}\right)=\mathfrak{F}_{1}$. Since $Z_{1} \notin \boldsymbol{D}(\mathfrak{I})$, we get that $\mathbb{F}=\left\langle Z_{1}\right\rangle \times$ (F* for some group $\mathfrak{F}^{*} \supset \mathfrak{F}_{1}$. Thus, 3 is the only minimal normal subgroup of 5 which is contained in $\boldsymbol{D}(\mathfrak{F})$, so 3 char (5), against Lemma 11.1. So suppose $Z_{1}$ does not centralize $\mathscr{S}_{1}$. Then $\mathfrak{K}_{1}$ has generators $H_{11}, H_{12}$ such that $H_{11}^{Z_{1}}=H_{11}$, $H_{12}^{Z_{1}}=H_{12}^{-1}$, and $C_{\mathfrak{p}}\left(Z_{1}\right)=\left\langle H_{11}\right\rangle \cdot\left\langle H_{12} H_{21} H_{22}\right\rangle$. Since $\mathfrak{I} / \mathfrak{S}$ is a four-group, it follows that $\bar{\Re}$ contains an element $\bar{X}$ which inverts $\overline{\mathfrak{Q}}$, so that $\Re=\left\langle Z_{1}, Z, X\right\rangle, X^{2} \in 3$ and $X \in N\left(\mathscr{S}_{1}\right)$. Also $\left\langle Z_{1}, Z\right\rangle \triangleleft \Omega$, so that $\left\langle H_{11}\right\rangle=C_{\mathfrak{\xi}_{1}}\left(\left\langle Z, Z_{1}\right\rangle\right) \triangleleft \Re$. As $\bar{X}$ inverts $\overline{\mathfrak{D}}$, it follows that $X$ inverts $H_{11}$ and that $H_{12}^{X}=H_{12} H_{11} Z^{e}$, where $e=0$ or 1 . As $\left\langle H_{21} H_{22}\right\rangle$ is the only subgroup of $\mathfrak{S}_{2}$ of order 4 which is inverted by $Z_{1}$, it follows that $X$ inverts $H_{21} H_{22}$, and that $H_{21}^{X}=H_{22} Z^{f}, f=0$ or 1 . Replacing $H_{11}$ by $H_{11}^{-1}$ if necessary we may assume that $e=0$.

Suppose $X^{-1} Z_{1} X \neq Z_{1}$. Since $\Omega$ is of order 8 , we get that

$$
X^{-1} Z_{1} X=Z_{1} Z
$$

so that $X H_{12}$ centralizes $Z_{1}$. Hence $X H_{12}$ is an element of 5 of order 8 , so that $3=\sigma^{2}(\mathfrak{F})$ char (5. This is not the case, so $X$ centralizes $Z_{1}$, so that $\widetilde{F}=\left\langle Z_{1}, X, H_{11}, H_{12} H_{21} H_{22}\right\rangle$. We now get

$$
X^{-1} H_{11} X=H_{11}^{-1}, X^{-1} H_{12} H_{21} H_{22} X=H_{12} H_{11} H_{22} Z^{f} \cdot H_{21} Z^{f}=H_{11} H_{12} H_{21} H_{22} \text {. }
$$

Hence $\mathfrak{G}^{\prime}=\left\langle H_{11}\right\rangle$, so that 3 char $\mathbb{F}$. This contradiction shows that this case does not occur.

Case (b)(ii). $\mathfrak{I} / \mathfrak{K}$ is a four-group and $\boldsymbol{C}_{\mathfrak{F}}\left(Z_{1}\right)$ is elementary.

Suppose $C_{\mathfrak{5}}\left(Z_{1}\right)$ is not a four-group. Then $C_{\mathfrak{5}}\left(Z_{1}\right)$ is elementary of order 8, by (11.2). Since $C_{\mathfrak{F}}\left(Z_{1}\right)=C_{\mathfrak{g}}\left(\left\langle Z, Z_{1}\right\rangle\right)$ and since $\left\langle Z, Z_{1}\right\rangle \triangleleft \Re$, it follows that $C_{\mathfrak{g}}\left(Z_{1}\right) \triangleleft \mathfrak{I}$, against $2 \in \pi_{2}$. Hence, $C_{\mathfrak{g}}\left(Z_{1}\right)$ is a fourgroup, so by (11.2), $Z_{1}$ inverts $\overline{\mathcal{D}}$. Since $\left\langle Z, Z_{1}\right\rangle \triangleleft \Re$, we get

$$
\mathfrak{S}_{\mathfrak{F}}\left(Z_{1}\right)=\mathfrak{W},
$$

by uniqueness of $\mathfrak{W}$. 
Let $\mathfrak{F}_{1}=\left\langle Z_{1}, \mathfrak{W}\right\rangle$ so that $\mathfrak{F}_{1}$ is elementary of order 8 and $\mathfrak{F}_{1} \leqq \mathfrak{F}_{\text {. }}$ Since $Z_{1}$ inverts $\overline{\mathfrak{D}}$, there are generators $H_{i 1}, H_{i 2}$ of $\mathfrak{S}_{i}$ such that $H_{i 1}^{Z_{1}}=H_{i 2}, i=1,2$. Hence $\mathfrak{W}=\left\langle Z, H_{11} H_{12} H_{21} H_{22}\right\rangle$, and

$$
\boldsymbol{N}_{\mathfrak{5}}\left(\mathfrak{F}_{1}\right)=\left\langle\mathfrak{W}, H_{11} H_{21}, H_{11} H_{12}\right\rangle,
$$

so that $\boldsymbol{A}_{\mathfrak{Y}}\left(\mathfrak{F}_{1}\right)=\boldsymbol{A}(\mathscr{C})$ where $\mathscr{C}: \mathfrak{F}_{1} \supset \mathfrak{W} \supset 1$. On the other hand, $\Re$ normalizes $\mathfrak{F}_{1}$, since $\left\langle Z_{1}, Z\right\rangle \triangleleft \Re$ and $\mathfrak{W}=C_{\mathfrak{p}}\left(\left\langle Z_{1}, Z\right\rangle\right)$. Let $X$ be an element of $\AA-\left\langle Z, Z_{1}\right\rangle$. Then $X^{-1} Z_{1} X=Z_{1} Y$ with

$$
Y \in \Re \cap \mathfrak{F}_{1} \cap \mathfrak{S}=\langle Z\rangle .
$$

If $\boldsymbol{C}_{\mathfrak{\xi}}(X)$ is elementary of order 8 , then since $\langle X, Z\rangle \triangleleft \Re$, we get $\boldsymbol{C}_{\mathfrak{S}}(X) \triangleleft \mathfrak{T}$, against $2 \in \pi_{2}$. Hence, $\boldsymbol{C}_{H}(X)$ is not elementary of order 8. Since $X^{2} \in\langle Z\rangle$, it follows that $X$ normalizes $\mathscr{F}_{1}$ and $\mathscr{S}_{2}$ and induces an inner automorphism of precisely one of $\mathfrak{F}_{1}, \mathfrak{F}_{2}$. Hence, $X$ does not centralize $\mathfrak{W}$. This implies that $\left|\boldsymbol{A}_{\mathfrak{\Sigma}}\left(\mathfrak{F}_{1}\right)\right|=8$, and that $\boldsymbol{C}_{\mathfrak{\Sigma}}\left(\mathfrak{F}_{1}\right)=\mathfrak{F}_{1}$. Hence, $\boldsymbol{C}_{\Theta}\left(\mathfrak{F}_{1}\right)=\mathfrak{F}_{1} \times \mathfrak{F}$, where $|\mathfrak{F}|$ is odd.

Since $\left|\boldsymbol{A}_{\mathfrak{x}}\left(\mathfrak{F}_{1}\right)\right|=8$, there is $E$ in $\mathfrak{F}$ such that $E \notin \boldsymbol{C}(\mathfrak{W})$. Thus, $\left\langle E, \mathfrak{F}_{1}\right\rangle$ is of order 16 and is the direct product of $\left\langle Z_{1}\right\rangle$ with $\langle\mathfrak{B}, E\rangle$, a dihedral group of order 8. If $|\mathfrak{F}|>16$, then $|\mathfrak{F} \cap \mathfrak{S}|>4$. This is not the case, so $\mathbb{F}=\left\langle E, \mathfrak{F}_{1}\right\rangle$. This violates Lemma 11.1 .

Case (c). $\mathfrak{I} / \mathfrak{F}$ is dihedral of order 8.

We first determine $\mathfrak{I}$ and $\mathfrak{I}_{0}=\boldsymbol{C}_{\mathfrak{x}}(\mathfrak{W})$. Let

$$
\Re_{1}=\Re \cap N\left(\Re_{1}\right)=\Re \cap N\left(\Re_{2}\right), \Re_{3}=\Re \cap N\left(\Re_{3}\right)=\Re \cap N\left(\Re_{4}\right) .
$$

Since $\Re / \mathbb{Z} \cong \mathfrak{I} / \mathscr{S}_{\mathcal{E}}$, it follows that $\Re_{i} / \mathbb{3}$ is a four-group, $i=1,3$. Choose $K_{i} \in \Re_{i}-3$ such that $\bar{K}_{i}$ centralizes $\bar{\Re}_{i}, i=1,3$, and set $K=K_{1} K_{3}$. Define $J=K^{2}$. Since $\Re_{i} / 3$ is a four-group, we get

$$
K_{i}^{2}=Z^{a_{i}}, \quad a_{i}=0 \text { or } 1, i=1,3 .
$$

Since $\bar{K}_{1}$ centralizes $\bar{\Re}_{1}$ and inverts $\bar{\Re}_{2}$, it follows that

$$
\boldsymbol{C}_{\mathfrak{5}}\left(K_{1}\right)=\mathfrak{S}_{2} ;
$$

and also that $\mathfrak{S}_{1}$ is generated by $H_{11}, H_{12}$ such that

$$
K_{1}^{-1} H_{1 i} K_{1}=H_{1,3-i}, \quad i=1,2 \text {. }
$$

Define $H_{2 i}$ by

$$
K_{3}^{-1} H_{1 i} K_{3}=H_{2 i}, \quad i=1,2,
$$

so that $\left\langle H_{21}, H_{22}\right\rangle=\mathfrak{K}_{2}$ and

$$
K_{3}^{-1} H_{2 i} K_{3}=H_{1 i} \text {. }
$$


We find that $\mathfrak{W}=\left\langle I_{1}, Z\right\rangle$, where $I_{1}=H_{11} H_{12} H_{21} H_{22}$, and

$$
\begin{gathered}
\mathfrak{I}_{0}=\left\langle H_{11} H_{12}, H_{21} H_{22}, H_{11} H_{21}, K_{3}, J, H_{11} K_{1}\right\rangle, \\
\mathfrak{T}_{0}^{\prime}=\left\langle H_{11} H_{12}\right\rangle \times\left\langle J H_{11} H_{21}\right\rangle .
\end{gathered}
$$

It is important to show that $I_{1} \ngtr Z$. Suppose false. First, suppose that $J$ is an involution.

We compute that $H_{11} H_{21}$ inverts $\mathfrak{I}_{0}^{\prime}$, so that $\mathfrak{I}_{0}^{\prime} H_{11} H_{21}$ contains only involutions. Also,

$$
\tilde{\mathfrak{I}}=\left\langle\mathfrak{I}_{0}^{\prime}, H_{11} H_{21}\right\rangle \operatorname{char} \mathfrak{I}_{0},
$$

since $\mathfrak{I}_{0}^{\prime}$ char $\mathfrak{I}_{0}$, and $\mathfrak{I}_{0}^{\prime} H_{11} H_{21}$ is the set of all elements of $\mathfrak{I}_{0}$ which invert $\mathfrak{T}_{0}^{\prime}$. Finally, $\tilde{\mathfrak{I}}$ contains exactly four elementary subgroups of order 8 ,

$$
\begin{aligned}
& \mathfrak{\mho}_{1}=\left\langle\mathfrak{W}, H_{11} H_{21}\right\rangle, \\
& \mathfrak{\mho}_{2}=\left\langle\mathfrak{W}, H_{12} H_{21}\right\rangle, \\
& \widetilde{\mho}_{3}=\langle\mathfrak{W}, J\rangle, \\
& \mathfrak{F}_{4}=\left\langle\mathfrak{W}, J H_{11} H_{12}\right\rangle .
\end{aligned}
$$

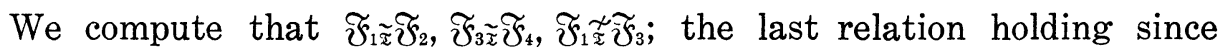
$\widetilde{F}_{1} \subset \mathfrak{S}_{2}, \widetilde{F}_{3} \nsubseteq \mathfrak{F}_{\text {. }}$

Suppose by way of contradiction that $I_{1 \dddot{\Im}} Z$. Let $\mathfrak{I}^{*}$ be a $S_{2^{-}}$ subgroup of $C_{\mathfrak{B}}\left(I_{1}\right)$ which contains $\mathfrak{I}_{0}$. Let $\mathbb{R}=\left\langle\mathfrak{T}, \mathfrak{T}^{*}\right\rangle$ so that $\mathfrak{I}_{0} \triangleleft$ $\mathbb{2}$ and $\mathbb{R}$ permutes $\mathfrak{W}^{\sharp}$ transitively. Since $\left\langle\mathfrak{I}_{0}^{\prime}, H_{11} H_{12}\right\rangle$ char $\mathfrak{I}_{0},\left\{\mathfrak{F}_{i} \mid 1 \leqq\right.$ $i \leqq 4\}$ is invariant under $\mathbb{2}$. Since $6=\left|\mathbb{2}: \mathfrak{I}_{0} \boldsymbol{C}_{\mathbb{R}}\left(\mathfrak{I}_{0}\right)\right|$, it follows that a $S_{2^{\prime}}$-subgroup $\mathfrak{L}_{2^{\prime}}$ of $\mathbb{Z}$ normalizes $\mathfrak{F}_{i}$ for some $i$. Thus, the orbit of $\mathfrak{F}_{i}$ under $\mathbb{R}$ is the orbit of $\widetilde{F}_{i}$ under $\mathfrak{I}$, so has two elements. Hence $\mathbb{R}_{2}$ normalizes every $\mathfrak{F}_{i}$, so $\mathfrak{R}_{2^{\prime}}$ centralizes $\mathfrak{F}_{i} / \mathfrak{M}, 1 \leqq i \leqq 4$. Since the $\mathfrak{F}_{i}$ generate $\overline{\mathfrak{I}}$, we get $\left[\overline{\mathfrak{T}}, \mathfrak{R}_{2^{\prime}}\right] \subseteq \mathfrak{W}$. In particular, $\left[\mathfrak{I}_{0}^{\prime}, \mathfrak{R}_{2^{\prime}}\right] \subseteq \mathfrak{W}=\boldsymbol{D}\left(\mathfrak{I}_{0}^{\prime}\right)$. Hence $\mathbb{R}_{2^{\prime}}$ centralizes $\mathfrak{I}_{j}^{\prime}$. This contradiction shows that $I_{1} \nsim Z$.

We may now assume that $J^{2}=Z$. Let $J_{0}=J H_{11} K_{1}$. Then $J_{0}$ inverts $\mathfrak{I}_{0}^{\prime}$, and $C_{\mathfrak{x}}\left(\mathfrak{T}_{0}^{\prime}\right) \cong \mathfrak{I}_{0}^{\prime} Z_{4} \times Z_{4}$. Let $\mathfrak{T}^{*}$ be a $S_{2}$-subgroup of $C\left(I_{1}\right)$ which contains $T_{0}$. Let $\mathbb{L}=\left\langle\mathfrak{I}, \mathfrak{I}^{*}\right\rangle \subseteq N\left(\mathfrak{I}_{0}\right)$. Thus, normalizes $\mathfrak{W}$, and $\mathbb{R}$ permutes transitively $\mathfrak{M}$. Since $J_{0} \mathfrak{T}_{0}^{\prime}$ is the set of all elements of $\mathfrak{I}_{0}$ which invert $\mathfrak{I}_{0}^{\prime}$, it follows that $L$ normalizes $J_{0} \mathfrak{I}_{0}^{\prime}$, so normalizes $\left\{J_{0}^{2}\right\}=\left\{X^{2} \mid X \in J_{0} \mathfrak{I}_{0}^{\prime}\right\}$. Hence, $J_{0}$ is an involution. We compute that $\mathfrak{I}_{0}$ permutes transitively $J_{0} \mathfrak{I}_{0}^{\prime}$, and so $J_{0} \mathfrak{T}_{0}^{\prime}=c c l_{\bar{x}}\left(J_{0}\right)$ is of cardinal $2^{4}$. Furthermore, there is a $S_{3}$-subgroup $\mathfrak{F}$ of $\mathbb{Q}$ which centralizes $J_{0}$. Suppose $J_{0} \sim Z$. Let $\mathfrak{F}=\left\langle J_{0}, \mathfrak{W}\right\rangle$. Then $[\mathfrak{F}, \mathfrak{P}]=\mathfrak{W}$, and so $\mathfrak{W} \subseteq \boldsymbol{O}_{2^{\prime}, 2}\left(C\left(J_{0}\right)\right)$. This implies that $\boldsymbol{A}_{\circledast}(\mathfrak{F})$ contains $\boldsymbol{A}(\mathscr{C})$, where $\mathscr{C}: \mathfrak{F} \supset\left\langle J_{0}\right\rangle \supset 1$. Since $\mathfrak{F} \triangleleft \mathfrak{F}^{\prime}$, it follows that $\boldsymbol{A}_{\Theta}(\mathfrak{F})$ is transitive on $\mathfrak{F}$, and so $N(\mathfrak{F})$ is non solvable. This is false, and so 


$$
J_{0} \nsim Z \text {. }
$$

Since $J^{2}=Z$, JFE contains no involutions. On the other hand, $\left\langle\mathfrak{F}, J, K_{3}\right\rangle$ is of index 2 in $\mathfrak{I}$, and so $J_{0}$ is conjugate to some element of $\left\langle\mathfrak{S}, J, K_{3}\right\rangle$. Since $\left(\mathfrak{S} K_{3}\right)^{K_{1}}=\mathfrak{S} K_{3} J$, it follows that $\mathfrak{S C}_{3}$ contains an involution. Since $\mathfrak{S}_{1}^{K_{3}}=\mathfrak{S}_{2}$ and $K_{3}^{2} \in\langle Z\rangle$, it follows that $K_{3}$ is an involution. Also, all involtions of $\mathfrak{S}_{2} K_{3}$ are conjugate under $\mathfrak{S}_{\mathfrak{C}}$ to $K_{3}$ or $K_{3} Z$. Since $K_{3}^{K_{1}}=K_{3} J^{ \pm 1}, K_{3}$ inverts $J$. Hence, $J^{-1} K_{3} J=K_{3} Z$, so all involutions of $\mathfrak{S}_{2} K_{3}$ are conjugate to $K_{3}$, whence are conjugate to $J_{0}$, so are not conjugate to $Z$.

By $(11.2)^{\prime}, \mathfrak{I}-\mathfrak{F}_{\mathfrak{C}}$ contains a conjugate of $Z$. Since $\mathfrak{S}_{\mathfrak{C}} J_{0} \cup \mathfrak{S}_{\mathcal{C}} J_{0} J \cup$ $\mathfrak{S}_{\mathfrak{C}} K_{3} \cup \mathfrak{S}_{\mathfrak{C}} K_{3} J$ contains all the involutions of $\mathfrak{I}-\mathfrak{S}$, and since $\left(\mathscr{S}_{\mathfrak{C}} J_{0}\right)^{K_{3}}=$ $\mathfrak{S}_{0} J_{0} J$, it follows that $\mathfrak{S}_{2} J_{0}$ contains a conjugate of $Z$. Now $\operatorname{ccl}_{\mathfrak{u}}\left(J_{0}\right)$ has cardinal $2^{4}$, and so $c c l_{\mathfrak{z}}\left(J_{0}\right) \cap \mathfrak{S}_{\mathcal{C}} J_{0}$ has cardinal $2^{3}$. By (11.2), $J_{0}$ induces an outer automorphism of precisely one of $\mathfrak{S}_{1}, \mathfrak{K}_{2}$, and also $\mathfrak{W} \subseteq C\left(J_{0}\right)$. It follows that $C_{\mathfrak{F}}\left(J_{0}\right)$ is dihedral of order $2^{3}$, and that $\mathfrak{S}_{2} J_{0}$ contains precisely 12 involutions. Hence, $\mathfrak{S}_{2} J_{0}$ contains precisely 4 conjugates of $Z$. Hence, $c c l_{\bar{\Sigma}}\left(Z_{1}\right)$ has cardinal $2^{3}$. Thus, $|\mathfrak{F}|=2^{5}$, and $|\mathfrak{F} \cap \mathfrak{S}|=$ $2^{3}$. We argue that every involution of $\mathbb{E}$ is contained in $\left\langle Z_{1}\right\rangle \times \mathbb{F} \cap \mathfrak{F}$. If not, $\mathbb{F}$ has a 4-group $\mathfrak{F}_{1}$ which is a complement to $\mathfrak{F} \cap \mathfrak{F}$, and so $\mathfrak{S C}_{\mathfrak{C}} \supset \mathfrak{S}_{1} J$, against the fact that $\mathfrak{S}_{\mathcal{C}} J$ has no involutions. By $(11.2)^{\prime}$, $\mathfrak{F} \cap \mathfrak{F}$ is not elementary, and so $\langle Z\rangle$ char $(\mathfrak{F}$, against Lemma 11.1. This contradiction shows that $I_{1} \nsim Z$.

Since $|\mathfrak{I}|>2^{5}$, Lemma 11.7(a) holds. Suppose $G \in \mathbb{B}$ and $\mathfrak{B}_{1}=$ $\mathfrak{W}^{G} \subset \mathfrak{I}$. We will show that $G \in \mathfrak{R}$. If $Z \in \mathfrak{W}_{1}$, then $Z^{G^{-1}} \in \mathfrak{W}$, so

$$
Z^{G^{-1}}=Z, G \in \mathfrak{N} .
$$

We may assume that $Z \notin \mathfrak{W}_{1}$. Set $Z^{\prime}=Z^{G}$. By Lemma 11.7(a), we get that

$$
X \in \mathfrak{W}_{1}-\left\langle Z^{\prime}\right\rangle \text { implies } C_{\mathfrak{x}}(X) \subseteq C_{\check{x}}\left(Z^{\prime}\right) \text {. }
$$

Now (11.10) and (11.2) imply that $\mathfrak{M}_{1} \cap \mathfrak{S}=1$. Let $\mathfrak{I}^{*}$ be a $S_{2}$-subgroup of $C_{\Theta}\left(Z^{\prime}\right)$ which contains $C_{\tilde{\Sigma}}\left(Z^{\prime}\right)$. Since some element of $\mathfrak{W}_{1}^{*}$ centralizes $\mathfrak{W}$, (11.10) implies that $\mathfrak{W} \subseteq C_{\Sigma}\left(Z^{\prime}\right)$. Hence

$$
\left[\mathfrak{W}, \mathfrak{W}_{1}\right] \subseteq \mathfrak{S} \cap \boldsymbol{O}_{2^{\prime}, 2}\left(C\left(Z^{\prime}\right)\right) \text {. }
$$

If $\left[\mathfrak{W}, \mathfrak{W}_{1}\right]=\langle Z\rangle$, then $Z \in \boldsymbol{O}_{2^{\prime} 2}\left(\boldsymbol{C}\left(Z^{\prime}\right)\right)$, against $I \not Z$. Hence $\left[\mathfrak{W}_{,} \mathfrak{W}_{1}\right]=1$, and

$$
\left\langle\mathfrak{W}, \mathfrak{W}_{1}\right\rangle=\mathfrak{W} \times \mathfrak{W}_{1} .
$$

Since $\mathfrak{I} / \mathfrak{F}_{\mathfrak{C}}$ is a dihedral group of order 8 , and since $\mathfrak{K} \cap \mathfrak{W}_{1}=1$, we can choose $W_{1}$ in $\mathfrak{W}_{1}$ such that $\mathfrak{S} W_{1}$ is the central involution of $\mathfrak{I} / \mathfrak{F}$. Then $\bar{W}_{1}$ inverts $\overline{\mathscr{G}} \overline{\mathcal{F}} / \overline{\mathscr{F}}$, so by Lemma $5.36, \bar{W}_{1}$ inverts some $S_{2^{\prime}}$ - 
subgroup of $\overline{\mathfrak{R}}$, which we may assume is $\overline{\mathfrak{Q}}$. With this normalization, we get $W_{1} \in \Re$, so that $\left\langle W_{1}, Z\right\rangle$ is a normal four-subgroup of $\Re$. Since $|\Re|=2^{4}, \Re$ is not of maximal class, so $W_{1} \in Z(\Re)$. Also, since $W_{1}$ inverts $\overline{\mathfrak{D}}$, (11.2) gives $\mathfrak{W}=C_{\mathfrak{5}}\left(W_{1}\right)$. If $W_{1}=Z^{\prime}$, then by (11.2), (11.10) is violated. Hence $W_{1} \neq Z^{\prime}$, so $\Omega$ centralizes $\mathfrak{W}_{1}$, by (11.10). This is also impossible since $\overline{\mathfrak{V}}_{1}$ is a four-subgroup of $\overline{\mathfrak{N}}$ which satisfies

$$
\overline{\mathfrak{S}} \cap \overline{\mathfrak{W}}_{1}=1,
$$

so that $\overline{\mathfrak{W}}_{1} \overline{\mathfrak{F}} / \overline{\mathfrak{F}}$ is not central in $\overline{\mathfrak{I}} / \overline{\mathfrak{F}}$. We conclude that whenever $G \in \mathbb{S}$ and $\mathfrak{W}^{G} \subseteq \mathfrak{I}$, then $G \in \mathfrak{R}$.

We now use the element $Z_{1}$ given in $(11.2)^{\prime}$. Suppose first that $C_{\mathfrak{F}}\left(Z_{1}\right)$ contains a four-group $\tilde{\mathfrak{W}}$ with $Z \in \tilde{\mathfrak{W}}$. We can then choose $N$ in $N(\mathfrak{S})$ such that $\tilde{\mathfrak{B}}=\mathfrak{W}^{N}$. Let $\mathfrak{I}^{*}$ be a $S_{2}$-subgroup of $C_{\mathbb{S}}\left(Z_{1}\right)$ which contains (5. Choose $G$ in (5) such that $\mathfrak{I}^{* a}=\mathfrak{I}$. Then $\tilde{\mathfrak{B}}^{a} \subseteq \mathfrak{I}$, so that $N G \in \mathfrak{R}$. Since $N(\mathfrak{C}) \subseteq \Re$, we get $G \in \Re$. This is not the case, since $Z_{1}$ is not contained in the center of any $S_{2}$-subgroup of $\Re$. Hence $C_{\mathfrak{5}}\left(Z_{1}\right)$ contains no four-group. By (11.2), $C_{5}\left(Z_{1}\right)$ contains an element $T$ of order 4 . Again, let $\mathfrak{I}^{*}$ be a $S_{2}$-subgroup of $C_{\mathscr{\leftrightarrow}}\left(Z_{1}\right)$ which contains (अ. Then $Z=T^{2} \in \boldsymbol{D}\left(\mathfrak{I}^{*}\right)$, so that $Z$ centralizes $\mathfrak{S}^{*}$, the unique element of $\mathscr{L}\left(\mathfrak{I}^{*}\right)$. Hence $\mathfrak{W}^{*} \subseteq \mathfrak{R}$, we get $\mathfrak{W}^{*}=\mathfrak{W}^{N}$ for some $N$ in $\mathfrak{R}$. Since $Z_{1} \in \mathfrak{S}^{*}$, we get $Z_{1} \in \mathfrak{W}^{N}$, so that $Z_{1} \subseteq \mathfrak{S}_{\boldsymbol{O}^{\prime}}(\mathfrak{R})$. This is not the case since $Z_{1} \in \mathfrak{I}-\mathfrak{H}$. With this contradiction we conclude that the width of $\mathfrak{S}$ is at most 1 , so the width of $\mathfrak{S E}$ is 1 .

Suppose $\mathfrak{S}=\mathfrak{I}$. In this case, we again get $3 \operatorname{char} C_{\mathfrak{z}}(J)$ for every involution $J$ of $\mathfrak{T}$, against Lemma 11.1 and (11.1). Hence, $\mathfrak{F} \subset \mathfrak{I}$. Since the width of $\mathfrak{F}_{\mathcal{C}}$ is 1 , we get that $\left|\mathfrak{T}: \mathfrak{F}_{\mathfrak{Z}}\right|=2$, and that $\mathfrak{S}_{\mathcal{C}}$ is the central product of $\mathscr{K}_{1}$ and $\mathfrak{S}_{2}$, where $\mathscr{F}_{1}$ is a quaternion group and $\mathfrak{S}_{2}$ is either cyclic or of maximal class.

Case 1. $\quad \mathfrak{S}_{2}$ is of maximal class.

Let $\tilde{\mathfrak{F}}_{2}$ be the unique cyclic subgroup of index 2 in $\tilde{S}_{2}$. Thus $\left|\tilde{\mathcal{F}}_{2}\right| \geqq 8$ and $\mathscr{S}_{1} \tilde{\mathcal{F}}_{2} \triangleleft \mathfrak{T}$. Since $|\mathscr{K}(\mathfrak{T})|=1$, we have $\mathfrak{W} \subseteq \mathfrak{S}_{1} \tilde{\mathcal{F}}_{2}$. Suppose $Z_{1} \in \mathfrak{F}_{1} \tilde{\mathcal{F}}_{2}$ and $Z_{1}$ is conjugate to $Z$ in (S). Then $Z$ is the unique involution in $\sigma^{2}\left(C_{\Sigma}\left(Z_{1}\right)\right)$, so $Z_{1}=Z$. Since $\mathfrak{T} / \mathscr{S}_{1} \tilde{\mathcal{F}}_{2}$ is a four-group, it follows that $\boldsymbol{D}(\mathfrak{T}) \leqq \mathfrak{\mathcal { S }}_{1} \tilde{\mathfrak{F}}_{2}$. Suppose $Z_{1} \in \mathfrak{F}_{\mathcal{C}}, Z_{1 \widetilde{ฒ}} Z$. Then $C_{\mathfrak{5}}\left(Z_{1}\right)$ contains an element $T$ of order 4 . Let $\mathfrak{I}^{*}$ be a $S_{2}$-subgroup of $C_{\circledast}\left(Z_{1}\right)$ which contains $\boldsymbol{C}_{\mathfrak{\Sigma}}\left(Z_{1}\right)$. Then $T \in \mathfrak{I}^{*}$, so $Z=T^{2} \in \boldsymbol{D}\left(\mathfrak{I}^{*}\right)$. By the preceding argument applied to $\mathfrak{I}^{*}$, we get $Z=Z_{1}$. Thus, 3 is weakly closed in $\mathfrak{S}$.

Choose $Z_{1} \in \mathfrak{I}, Z_{1} \neq Z, Z_{1} \sim Z$. Thus, $\mathfrak{I}=\mathfrak{S}_{\mathfrak{C}}\left\langle Z_{1}\right\rangle$. Let

$$
\mathfrak{F}=C_{\mathfrak{x}}\left(Z_{1}\right)=\left\langle Z_{1}\right\rangle \times C_{\mathfrak{5}}\left(Z_{1}\right) \text {. }
$$

By Lemma 11.1, $\mathfrak{F}$ is elementary, so $|\mathfrak{F}|=8$. It follows that $\boldsymbol{A}_{\mathfrak{\Sigma}}(\mathfrak{F})$ 
contains $\boldsymbol{A}(\mathscr{C})$ where $\mathscr{C}: \mathfrak{F} \supset \mathfrak{B} \supset 1$, and so $\boldsymbol{A}_{\mathscr{G}}(\mathfrak{F})$ is nonsolvable.

Case 2. $\quad \mathfrak{S}_{2}$ is cyclic of order $\geqq 8$.

Here, Lemma 11.1 implies that 3 is weakly closed in $\mathfrak{F}$. Choose $Z_{1} \in \mathfrak{T}, Z_{1} \neq Z, Z_{1} \sim Z$, and let $H$ be a generator for $\mathscr{K}_{2}$. By Lemma 11.1, $C_{\mathfrak{g}}\left(Z_{1}\right)$ is elementary. Let $K$ be an element of $\mathfrak{S}_{2}$ of order 4 so that $Z_{1}$ inverts $K$. Also, $\mathfrak{S}_{1}$ has generators $H_{1}, H_{2}$ with $H_{1}^{Z_{1}}=H_{2}$. Hence $C_{\mathfrak{p}}\left(Z_{1}\right)=\langle Z\rangle \times\left\langle H_{1} H_{2} K\right\rangle$. Let

$$
\text { ⿷匚 }=C_{\tilde{\Sigma}}\left(Z_{1}\right)=\left\langle Z_{1}\right\rangle \times C_{\mathfrak{\xi}}\left(Z_{1}\right) \cdot
$$

It follows that $\left|N_{\mathfrak{x}}(\mathfrak{F})\right|=2^{5}$ and that $3=Z\left(N_{\mathfrak{x}}(\mathfrak{F})\right)$. Let $\mathfrak{I}^{*}$ be a $S_{2}$-subgroup of $C_{\circledast}\left(Z_{1}\right)$ which contains $\left(5\right.$. By symmetry, $\mid N_{\mathbb{x}^{*}}\left((\mathfrak{5}) \mid=2^{5}\right.$ and $\left\langle Z_{1}\right\rangle=\boldsymbol{Z}\left(\boldsymbol{N}_{\mathbb{R}^{*}}(\mathfrak{\xi})\right)$. This implies $\boldsymbol{N}_{\mathscr{\Theta}}(\mathfrak{\xi})$ is nonsolvable.

Case 3. $\left|\mathfrak{F}_{2}\right|=4$.

First, suppose there is $Z_{1} \in \mathfrak{I}-\mathfrak{K}, Z_{1} \sim Z$. Then $\mathscr{\mathcal { C }}_{1}$ has generators $H_{1}, H_{2}$ with $H_{1}^{Z_{1}}=H_{2}$. Let $H$ be a generator for $\mathfrak{\mathcal { F }}_{2}$. By Lemma 11.1, $C_{\check{x}}\left(Z_{1}\right)$ is elementary. Let $A=H_{1} Z_{1}$. Then $A$ has order 8. Also $H^{-1} A H=A^{5}, Z_{1} A Z_{1}=A^{3}$. Let $T=H_{1} H_{2} H$ so that $T$ is an involution and $Z_{1} T=T Z_{1}$. Thus, $\mathfrak{I}$ is the split extension of $\langle A\rangle$ by $\left\langle Z_{1}, T\right\rangle$ and $\langle A\rangle=C_{\mathfrak{z}}(A)$. By a result of Fong [14], $\mathfrak{I}$ is not the $S_{2}$-subgroup of any perfect group.

Choose $Z_{1} \in \mathfrak{T}, Z_{1} \neq Z, Z_{1} \sim Z$. By the preceding paragraph, $Z_{1} \in$ $\mathfrak{F}$. We may assume that $C_{\check{\tau}}\left(Z_{1}\right)$ is a $S_{2}$-subgroup of $\boldsymbol{C}_{\mathfrak{R}}\left(Z_{1}\right)$. Since 3 char $C_{\mathfrak{5}}\left(Z_{1}\right)$, it follows that $C_{\mathfrak{F}}\left(Z_{1}\right) \subset C_{\mathfrak{x}}\left(Z_{1}\right)$. Choose $T \in C_{\mathfrak{x}}\left(Z_{1}\right)-\mathfrak{S}$. We can then choose an element $H_{1}$ of $\mathfrak{F}_{1}$ such that $\mathfrak{F}_{1}=\left\langle H_{1}, H_{1}^{T}\right\rangle$. Let $H_{2}=H_{1}^{T}$. Then $H_{2}^{T}=H_{1} Z^{a}$ for some $a=0$ or 1 . Thus $Z_{1}=$ $H_{1} H_{2} H$, where $H$ is a generator for $\mathfrak{S}_{2}$ and $C_{\mathfrak{5}}\left(Z_{1}\right)=\left\langle H_{1} H_{2}, H\right\rangle$. Hence $C_{T}\left(Z_{1}\right)=\left\langle H_{1} H_{2}, H, T\right\rangle$ so that $C_{\tilde{x}}\left(Z_{1}\right)^{\prime} \subseteq 3$. By Lemma 11.1, $C_{\mathfrak{z}}\left(Z_{1}\right)$ is abelian. If $T^{2} \in\langle Z\rangle$, then $\langle Z\rangle=\boldsymbol{D}\left(\boldsymbol{C}_{\tilde{\Sigma}}\left(Z_{1}\right)\right)$, against Lemma 11.1. Hence $T^{2} \notin Z$. Since $T$ centralizes $H_{1} H_{2}$, we get $a=1$. By Lemma 11.1, $C_{\mathfrak{x}}\left(Z_{1}\right)$ is of type $(4,4)$. Now $H_{1} H=T_{0}$ is an involution of $\mathfrak{I}-C_{\mathfrak{z}}\left(Z_{1}\right)$, so $\mathfrak{I}=C_{\mathfrak{z}}\left(Z_{1}\right)\left\langle T_{0}\right\rangle$. Since $C_{\mathfrak{z}}\left(Z_{1}\right)$ is the direct product of $\langle T\rangle$ and $T_{0}^{-1}\langle T\rangle T_{0}$, the proof is complete.

LemmA 11.9. Suppose $|\mathscr{U}(\mathfrak{T})|=1$ and $\overline{\mathfrak{S}} \triangleleft \overline{\mathfrak{R}}$. Then $\mathfrak{W}=3$ and $\mathfrak{I} \subseteq N_{\mathscr{G}}(\mathfrak{I})^{\prime}$.

Proof. First, suppose $\mathfrak{W}=3$. In this case, it suffices to show that $\mathfrak{W}$ is weakly closed in $\mathfrak{I}$. Suppose $\mathfrak{W}_{1}=\mathfrak{W}^{a} \subset \mathfrak{T}, \mathfrak{W}_{1} \neq \mathfrak{W}$. Let $\mathscr{C}:\left\langle\mathfrak{W}_{1}, \mathfrak{W}\right\rangle \supset \mathfrak{W} \supset 1, \mathscr{C}^{*}:\left\langle\mathfrak{W}_{1}, \mathfrak{W}\right\rangle \supset \mathfrak{W}_{1} \supset 1$. Since $\mathfrak{W} \subseteq Z(\mathfrak{I})$, it follows that for each $W$ in $\mathfrak{S}^{\sharp}$, every 2-subgroup of $C(W)$ which contains $\mathfrak{W}$ centralizes $\mathfrak{W}$. Hence, $\mathfrak{W} \cap \mathfrak{W}_{1}=1$ and $\left|\boldsymbol{A}_{\circlearrowleft}(\mathscr{C})\right| \geqq 4,\left|\boldsymbol{A}_{\oplus}\left(\mathscr{C}^{*}\right)\right| \geqq 4$. This implies that $\boldsymbol{A}_{\circledast}\left(\left\langle\mathfrak{W}, \mathfrak{W}_{1}\right\rangle\right)$ is nonsolvable. 
We may now assume that $|3|=2$. First, suppose $I+Z$. With this assumption, we may show that $\mathfrak{W}$ is weakly closed in $\mathfrak{T}$. Suppose $\mathfrak{W}_{1}=\mathfrak{W}^{a} \subset \mathfrak{I}, \mathfrak{W}_{1} \neq \mathfrak{W}$. Let $\mathfrak{W}_{2}=\mathfrak{W}_{1} \cap \mathfrak{I}_{0}$. Since $\mathfrak{W}_{2} \neq 1$, it follows that $\left[\mathfrak{W}, \mathfrak{W}_{1}\right] \subseteq 3 \cap \mathfrak{Z}^{G}=1$. If $\mathfrak{W} \cap \mathfrak{M}_{1} \neq 1$, then $\mathfrak{W}=\mathfrak{W}_{1}$, by Lemma 11.7. Hence, suppose $\mathfrak{W} \cap \mathfrak{W}_{1}=1$. It follows that $\boldsymbol{A}_{\circlearrowleft}\left(\left\langle\mathfrak{W}, \mathfrak{W}_{1}\right\rangle\right)$ is nonsolvable. This is impossible, so $\mathfrak{W}$ is weakly closed in $\mathfrak{T}$. We next show that 3 is weakly closed in $\mathfrak{T}$. Suppose $Z_{1}=Z^{a} \in \mathfrak{I}, Z \neq Z_{1}$ by the previous argument, $Z_{1}$ does not centralize $\mathfrak{W}$. Hence, if $\mathfrak{U} \in$ $\mathscr{S} \mathscr{C} \mathscr{N}_{2}(\mathfrak{I})$, then $C_{\mathfrak{q}}\left(Z_{1}\right)$ is cyclic. If $\mathfrak{A}=\mathfrak{W}$, then $\mathfrak{I}$ is dihedral of order 8 , against $|\mathscr{U}(\mathfrak{T})|=1$. Hence $|\mathfrak{A}|>4$, so $\left|\boldsymbol{C}_{\mathfrak{H}}\left(Z_{1}\right)\right| \geqq 4$. This implies that $Z \in D\left(C_{\mathfrak{r}}\left(Z_{1}\right)\right)$. Thus, if $\widetilde{\mathfrak{I}}=\mathfrak{T}^{X}$ is a $S_{2}$-subgroup of $C_{\circledast}\left(Z_{1}\right)$ which contains $C_{\mathfrak{\Sigma}}\left(Z_{1}\right)$, then $Z$ centralizes $\mathfrak{B}^{x}$. Hence, $\mathfrak{W}^{X}=\mathfrak{W}^{N}$ for some $N$ in $\mathfrak{R}$, so $Z \in \mathfrak{W}^{x}$ and $\left\langle Z, Z_{1}\right\rangle=\mathfrak{W}^{x}$, against $I \not Z Z$.

Thus, if $I \ngtr Z$, then 3 is weakly closed in $\mathfrak{I}$. As this violates (11.1), we conclude that $I \sim Z$.

We will show that $\mathfrak{B}$ is weakly closed in $\mathfrak{T}$. Suppose

$$
\mathfrak{W}_{1}=\mathfrak{W}^{G} \subset \mathfrak{I},
$$

$\mathfrak{W}_{1} \neq \mathfrak{W}$. Then $\mathfrak{W} \cap \mathfrak{W}_{1}=1$ and $\left\langle\mathfrak{W}, \mathfrak{W}_{1}\right\rangle$ is abelian. Again we see that $\boldsymbol{A}_{\mathfrak{G}}\left(\left\langle\mathfrak{W}, \mathfrak{W}_{1}\right\rangle\right)$ is nonsolvable. Hence, $\mathfrak{W}$ is weakly closed in $\mathfrak{T}$. Finally, we show that $\mathfrak{W}$ is the weak closure of 3 in $\mathfrak{I}$. Proceeding as in the previous paragraph, we get that if $Z_{1}=Z^{G} \in \mathfrak{I}, Z_{1} \neq Z$, then $\mathfrak{S}^{X}=\left\langle Z, Z_{1}\right\rangle$ for some $X$ in $\mathbb{S}$, so $\mathfrak{W}^{X}=\mathfrak{W}^{N}$ for some $N$ in $\mathfrak{N}$, so $Z_{1} \in \mathfrak{I} \cap \mathfrak{W}^{N} O_{2^{\prime}}(\mathfrak{R}) \subseteq \mathfrak{W}$. Thus, $\mathfrak{W}$ is the weak closure of 3 in $\mathfrak{I}$.

By the proof of Theorem 14.4.1 of [19] applied to $N_{\mathbb{\leftrightarrow}}(\mathfrak{W})$, we

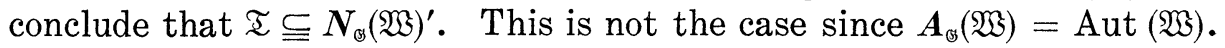
The proof is complete.

THEOREM 11.1. One of the following holds:

(a) $\mathfrak{T}$ is dihedral.

(b) $\mathfrak{I}=g p\left\langle A, B \mid A^{8}=B^{2}=1, B A B=A^{3}\right\rangle, i(\mathbb{S})=1$.

(c) $|\mathfrak{I}|=2^{6}$, $\mathfrak{I}$ has exactly 3 involutions, each of which is central and $i(\mathbb{S})=1$.

(d) $\mathfrak{I} \cong Z_{4} \zeta Z_{2}, i(\mathbb{S})=1$.

Proof. If $\mathscr{U}(\mathfrak{I})=\phi$, we have either (a) or (b) by Lemma 11.4(i). If $|\mathscr{C}(\mathfrak{T})|>1$, we have (a), by Lemma 11.4(ii).

We may assume that $|\mathscr{C}(\mathfrak{T})|=1$. Suppose $\overline{\mathfrak{S}} \triangleleft \overline{\mathfrak{R}}$. Then by Lemmas 11.9 and 5.28 , we have (c) if $\mathfrak{I}^{\prime} \neq 1$, while if $\mathfrak{T}^{\prime}=1$, we have (a) by [8]. Suppose $\overline{\mathfrak{B}} \sharp \overline{\mathfrak{N}}$. Then $\mathfrak{I} \cong Z_{4} \succ Z_{2}$ by Lemma 11.8. Also, $i(\mathbb{S})=1$ in this case, as the proof of Lemma 11.8 shows.

The proof is complete.

THEOREM 11.2. If $2 \in \pi_{2}$, then (S) is isomorphic to one of the 
following groups: $U_{3}(3), L_{3}(3), M_{11}, A_{7}, L_{2}(q)$.

To prove Theorem 11.2, we examine the possibilities of Theorem 11.1.

Suppose (a) holds. By the fundamental result of Gorenstein and Walter [19], we have $\mathbb{S} \cong L_{2}(q)$ for some $q$ or $\mathbb{S} \cong A_{7}$.

Suppose (b) holds. Let $\mathfrak{W}$ be a four-subgroup of $\mathfrak{I}$. (Since $\mathscr{U}(\mathfrak{T})=\varnothing$, this does not conflict with earlier notation). Suppose $p \geqq 5$. We will show that Hypothesis 6.1 is satisfied with $\mathfrak{B}$ in the role of $\mathfrak{A}, \pi=\{2\}$, and with $p$ in the role of $q$. Certainly, (6.1)(a), (b), (c) hold, as $\mathbb{B}$ is an $N$-group. As for $(6.1)(\mathrm{d})$, we see that every $\{2, p\}$-subgroup of $(5)$ is $p$-closed, so that $(6.1)(\mathrm{d})$ also holds. Thus, if $\mathfrak{\Omega}_{1}, \mathfrak{\Omega}_{2}$ are maximal elements of $И(\mathfrak{W} ; p)$ and if $C_{\mathfrak{Q}_{i}}(W) \neq 1$ for some $W$ in $\mathfrak{S}^{\sharp}$ and $i=1,2$, then by Lemma $6.2, \mathfrak{\Omega}_{1}$ and $\mathfrak{\Omega}_{2}$ are conjugate under $\boldsymbol{C}(\mathfrak{B})$.

We next argue that if $\mathfrak{\Omega}_{1}, \mathfrak{\Omega}_{2}$ are maximal elements of $И(\mathfrak{W} ; 3)$, then $\mathfrak{\Omega}_{1}$ and $\mathfrak{\Omega}_{2}$ are conjugate under $N_{\circledast}(\mathfrak{W})$. Suppose false. Among all pairs of maximal elements of $И(\mathfrak{B} ; 3)$ which lie in different orbits of $\boldsymbol{U}_{\mathscr{\Theta}}(\mathfrak{W})$, choose $\left(\mathfrak{\Omega}_{1}, \mathfrak{Z}_{2}\right)$ such that $\left|\mathfrak{\Omega}_{1} \cap \mathfrak{\Omega}_{2}\right|$ is maximal. Let $W$ be a fixed element of $\mathfrak{S}^{\sharp}$. As $\boldsymbol{A}_{\Theta}(\mathfrak{W})=\operatorname{Aut}(\mathfrak{W})$, it follows that there are $N_{1}, N_{2}$ in $N(\mathfrak{W})$ such that $W$ has nontrivial fixed points on $\mathfrak{D}_{i}^{N_{i}}$, $i=1,2$. Hence $\mathfrak{F}=\mathfrak{\Omega}_{1} \cap \mathfrak{\Omega}_{2} \neq 1$. Since $\mathfrak{\Omega}_{1}, \mathfrak{\Omega}_{2}$ are distinct maximal elements of $И(\mathfrak{W} ; 3)$, we have $\mathbb{F} \subset \mathfrak{Q}_{i}, i=1,2$. Let $\mathfrak{F}_{i}=N_{\mathfrak{D}_{i}}(\mathfrak{F})$, and let $\mathfrak{M}=N_{\mathfrak{\Theta}}(\mathfrak{F})$. Since $\mathfrak{M}$ is solvable, it follows that there is $X$ in $N_{\mathfrak{m}}(\mathfrak{B})$ such that $\left\langle\mathfrak{D}_{1}, \mathfrak{D}_{2}^{X}\right\rangle=\mathbb{F}$ is a 3-group. Let $\tilde{\mathfrak{D}}$ be a maximal element of $И(\mathfrak{W} ; 3)$ which contains $\left(\mathfrak{F}\right.$. Then $\tilde{\mathfrak{Q}} \cap \mathfrak{\Omega}_{1} \supseteq \mathfrak{D}_{1} \supset \mathfrak{D}$, so that $\widetilde{\mathfrak{Q}}$ and $\mathfrak{\Omega}_{1}$ are in the same orbit under $\boldsymbol{N}_{\Theta}(\mathfrak{W})$. Since

$$
\tilde{\mathfrak{D}} \cap \mathfrak{\Omega}_{2}^{X} \supseteqq \mathfrak{D}_{2}^{X} \supset \mathfrak{D}^{X},
$$

$\tilde{\mathfrak{Q}}$ and $\mathfrak{Q}_{2}^{X}$ are also in the same orbit under $N(\mathfrak{W})$. Thus, our assertion follows.

If $\subseteq$ is a solvable subgroup of $\mathbb{S}$ which contains $\mathfrak{I}$, then $\mathfrak{I} \nsubseteq \mathfrak{S}^{\prime}$. Hence, no nonidentity solvable subgroup of $\mathbb{B}$ has a normalizer which dominates both $\mathfrak{S}$ and $\mathfrak{M}$. By the preceding argument, we conclude that every involution of $\mathfrak{I}-3$ inverts some $S_{\{2,3\}}$-subgroup of $N$. We will show that every involution of $\mathfrak{I}-3$ inverts $\boldsymbol{O}_{2^{\prime}}(\mathfrak{R})$. Let $\mathfrak{R}_{3}$ be a $S_{3}$-subgroup of $\mathfrak{R}$ permutable with $\mathfrak{I}$. Choose $W$ in $\mathfrak{B}-3$ and let $\mathfrak{A}=\boldsymbol{C}_{\mathfrak{R}_{3}}(W)$. Let $\mathfrak{T}_{1}=\boldsymbol{N}_{\mathfrak{x}}(\mathfrak{W})$, so that $\mathfrak{I}_{1}$ is dihedral of order 8 . Suppose by way of contradiction that $\mathfrak{A} \neq 1$. Since $\mathfrak{A}$ is a $S_{3}$-subgroup of $C_{\mathscr{\Theta}}(\mathfrak{W})$, it follows that $N_{\mathbb{\Theta}}(\mathfrak{A})$ dominates $\mathfrak{W}$ and $N_{\mathscr{\Theta}}(\mathfrak{R}) \supset \mathfrak{I}_{1}$. Hence $\mathfrak{I}_{1}$ is a $S_{2}$-subgroup of $N_{\mathfrak{G}}(\mathfrak{R})$. This implies that $N(\mathfrak{U}) \cap \mathfrak{R} \subseteq \boldsymbol{O}_{2^{\prime}}(\mathfrak{R}) \mathfrak{I}$, since if $R$ is any element of $\mathfrak{R}-\boldsymbol{O}_{2^{\prime}}(\mathfrak{I})$ of odd order, then $\left\langle\mathfrak{I}_{1}, R\right\rangle$ contains a $S_{2}$-subgroup of $\mathfrak{R}$. Since $N(\mathfrak{U}) \cap \mathfrak{N} \subseteq O_{2^{\prime}}(\mathfrak{R}) \mathfrak{T}$, it follows 
that $|\mathfrak{R}: \mathfrak{A}|_{3} \geqq 9$. Let $\mathfrak{Q}$ be a $S_{2}$-subgroup of $N_{\mathscr{\Theta}}(\mathfrak{U})$ which contains $\mathfrak{A T}_{1}$. The preceding inequality implies that $\mathfrak{U} \subseteq \boldsymbol{O}_{3}(\mathbb{R})$. Hence,

$$
\left|\mathcal{L}: \boldsymbol{O}_{3}(\mathbb{R})\right|_{3}=3
$$

and $\mathfrak{W}$ is faithfully represented on $\boldsymbol{O}_{3}(\mathbb{Q})$. Enlarge $\mathbb{Q}$ to a maximal $\{2,3\}$-subgroup $\mathfrak{M}$ of $\mathfrak{S}$ and let $\mathfrak{P}$ be a $S_{3}$-subgroup of $\mathfrak{M}$. Since $\mathfrak{I}_{1}$ is a $S_{2}$-subgroup of $\mathfrak{M}$, it follows that $\boldsymbol{O}_{3}(\mathfrak{M})$ is a maximal element of $И(\mathfrak{W}: 3)$. By construction, $\boldsymbol{N}_{\circledast}\left(\boldsymbol{O}_{3}(\mathfrak{M})\right)$ dominates $\mathfrak{M}$, so by a previous argument, it follows that the maximal elements of $И(\mathfrak{W} ; 3)$ are permuted transitively by $C_{\mathscr{\Theta}}(\mathfrak{W})$. Since $\mathfrak{W}$ normalizes some $S_{3}$-subgroup of $\mathfrak{R}$, and since $\boldsymbol{C}_{\oplus}(\mathfrak{W}) \subseteq \mathfrak{N}$, it follows that $\boldsymbol{O}_{3}(\mathfrak{M})$ contains a $S_{3}$-subgroup of $\mathfrak{R}$. This is not the case, since $\mathfrak{I}_{1}$ normalizes no $S_{3}$-subgroup of $\mathfrak{R}$. Hence $\mathfrak{X}=1$, so every involution of $\mathfrak{R}-3$ inverts $\boldsymbol{O}_{2^{\prime}}(\mathfrak{R})$. This implies that $\mathfrak{R}$ has an abelian 2-complement. Hence $\left(\mathfrak{S} \cong L_{3}(3)\right.$ or $M_{11}$ by a result of Wong [45].

Suppose (c) holds.

Suppose by way of contradiction that $5 \| \boldsymbol{A}_{\Theta}(\mathfrak{I}) \mid$. Let $\mathfrak{R}_{5}$ be a $S_{5}$ subgroup of $\mathfrak{N}$ permutable with $\mathfrak{I}$ and let $\mathfrak{M}$ be a maximal $\{2,5\}$ subgroup of $\mathbb{S B}^{3}$ which contains $\mathfrak{T}_{\mathfrak{N}}$. Let $\mathfrak{M}_{5}$ be a $S_{5}$-subgroup of $\mathfrak{M}$ which contains $\mathfrak{N}_{5}$. Since $3 \boldsymbol{O}_{5}(\mathfrak{M}) / \boldsymbol{O}_{5}(\mathfrak{M})$ is central in $\mathfrak{M} / \boldsymbol{O}_{5}(\mathfrak{M})$, it follows that 3 normalizes $\mathfrak{M}_{5}$. Also, we see that

If $\subseteq$ is a solvable subgroup of $(S)$ which

$$
\text { contains } \mathfrak{M} \text {, then } 5 \| \subseteq: \Im^{\prime} \mid \text {. }
$$

To see this, observe that since $\mathfrak{M}$ is a maximal $\{2,5\}$-subgroup of $\mathbb{B}$, $\mathfrak{M}$ is a $S_{\{25\}}$-subgroup of $\mathfrak{S}$. The structure of $\mathfrak{I}$ forces the 2-length of $\subseteq$ to be one, so $N_{\mathfrak{S}}(\mathfrak{I})$ covers $\subseteq / \boldsymbol{O}_{2^{\prime}}(\mathfrak{S})$. Since $5 \| \boldsymbol{A}_{\mathfrak{G}}(\mathfrak{T}) \mid$, we get $5 \| \boldsymbol{A}_{\tilde{\subseteq}}(\mathfrak{T}) \mid$, by construction. Hence (11.12) holds.

Let $\widetilde{\mathfrak{M}}_{5}$ be a maximal element of $И(3 ; 5)$ which contains $\mathfrak{M}_{5}$. Let \& be a $S_{2}$-subgroup of $N\left(\widetilde{\mathfrak{M}}_{5}\right)$ which contains $3 \widetilde{\mathfrak{M}}_{5}$. Then $\widetilde{\mathfrak{M}}_{5}=\boldsymbol{O}_{5}(\mathbb{R})$, by maximality of $\widetilde{\mathfrak{M}}_{5}$. Also $3 \widetilde{\mathfrak{M}}_{5} / \widetilde{\mathfrak{M}}_{5}$ is central in $\mathbb{2} / \widetilde{\mathfrak{M}}_{5}$, so using the maximality of $\widetilde{\mathfrak{M}}_{5}$ once again, we get that $\widetilde{\mathfrak{M}}_{5}$ is a $S_{5}$-subgroup of $\mathbb{L}$. Thus, $\widetilde{\mathfrak{M}}_{5}$ is a $S_{5}$-subgroup of $\mathbb{S}$.

Case 1. 3 centralizes $\mathfrak{M}_{5}$.

Since $\mathfrak{M}$ is a maximal $\{2,5\}$-subgroup of $\mathbb{S}$, it follows that $\mathfrak{M}_{5}$ is a $S_{5}$-subgroup of $\boldsymbol{C}_{\mathscr{\Theta}}(Z)$ for all $Z$ in $Z^{\ddagger}$. Hence $\mathfrak{M}_{5}=\mathfrak{M}_{5} \cap C(Z)$ for all $Z$ in $3^{*}$. This implies that $\mathfrak{M}_{5}=\widetilde{\mathfrak{M}}_{5}$.

Since 3 centralizes $\mathfrak{M}_{5}=\widetilde{\mathfrak{M}}_{5}$, it follows that $N(3)$ covers $N\left(\mathfrak{M}_{5}\right)$ / $C\left(\mathfrak{M}_{5}\right)$. By (11.12), $5 \| N\left(\mathfrak{M}_{5}\right): N\left(\mathfrak{M}_{5}\right)^{\prime} \mid$. If $5 \in \pi_{1} \cup \pi_{2}$, then 0.3 .4 and a standard transfer argument show that $\mathfrak{M}_{5} \subseteq N\left(\mathfrak{M}_{5}\right)^{\prime}$. We conclude that $5 \in \pi_{3} \cup \pi_{4}$.

Suppose $5 \in \pi_{4}$. By Theorem 10.2, $\mathscr{A}(5) \subseteq \mathscr{C}^{*}(\mathbb{S})$. Hence $\mathfrak{M} \subseteq$ 
$\boldsymbol{M}\left(\mathfrak{M}_{5}\right)$. This violates (11.12).

Suppose $5 \in \pi_{3}$. Choose a prime $q$ such that a maximal element

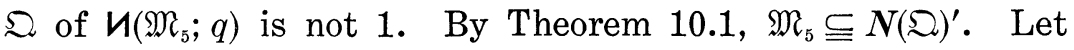

$$
\hat{\mathfrak{M}}_{5}=\mathfrak{M}_{5} \cap \boldsymbol{O}_{5^{\prime}}(\boldsymbol{N}(\mathfrak{Q})) \text {, }
$$

so that $\mathfrak{M}_{5} \subseteq N\left(\hat{\mathfrak{M}}_{5}\right)^{\prime}$. Since 3 centralizes $\hat{\mathfrak{M}}_{5}, 3$ is characteristic in some $S_{2}$-subgroup of $C\left(\hat{\mathfrak{M}}_{5}\right)$. Hence $\mathfrak{M}_{5} \subseteq N(3)^{\prime}$, against (11.12).

Case 2. 3 does not centralize $\mathfrak{M}_{5}$.

Since $3=\mathfrak{I}^{\prime}$ and since $\mathfrak{I} \mid\langle Z\rangle$ is extra special for all $Z$ in $Z^{\#}$, it follows that $\boldsymbol{O}_{5}(\mathfrak{M})$ contains an elementary subgroup of order $5^{3}$. Hence $5 \in \pi_{3} \cup \pi_{4}$ and $\mathfrak{M}$ contains an element of $\mathscr{A}(5)$. If $5 \in \pi_{4}$, then $\mathfrak{M} \subseteq$ $\boldsymbol{M}\left(\mathfrak{M}_{5}\right)$, by Theorem 10.2. This conflicts with (11.12), so $5 \in \pi_{3}$.

By Theorem 1 of [39],

$$
\mathfrak{M}=C_{\mathfrak{N}}\left(Z\left(\mathfrak{M}_{5}\right)\right) \cdot N_{\mathfrak{M}}\left(J\left(\mathfrak{M}_{5}\right)\right) \text {. }
$$

Since $\mathfrak{I} \boldsymbol{O}_{5}(\mathfrak{M}) / \mathfrak{T}^{\prime} \boldsymbol{O}_{5}(\mathfrak{M})$ is a chief factor of $\mathfrak{M}$, it follows that $\mathfrak{M}=$

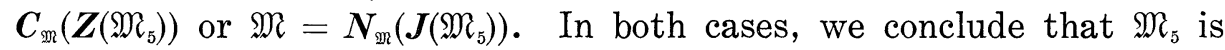
a $S_{5}$-subgroup of $\mathfrak{G}$.

Let $\Omega$ be a maximal element of $И\left(\mathfrak{M}_{5} ; q\right)$ with $\mathfrak{Q} \neq 1, q$ a prime. By Theorem 10.1, $\mathfrak{M}_{5} \subseteq N(\mathfrak{\Omega})^{\prime}$. By the transitivity theorem, $N(\mathfrak{Q})$ contains a $S_{2}$-subgroup of $(5$. This is not the case, by (11.12). We conclude that $5 \nmid\left|\boldsymbol{A}_{\mathscr{G}}(\mathfrak{T})\right|$.

Let $Z_{1}, Z_{2}, Z_{3}$ be the involutions of $\mathfrak{I}$. Since $i(\mathbb{S})=1$, these involutions are fused in $N(\mathfrak{T})$.

We will show that $S_{3}$-subgroups of $\boldsymbol{A}_{\circlearrowleft}(\mathfrak{I})$ are of order 3 . Let $\mathfrak{X}$ be a $S_{3}$-subgroup of $\boldsymbol{A}_{\oplus}(\mathfrak{T})$. Since $\mathfrak{I} / \mathfrak{I}^{\prime}$ is elementary of order $2^{4}$, we may assume that $\mathfrak{A}$ is elementary of order $3^{2}$. Then $\mathfrak{A}=\mathfrak{A}_{1} \times \mathfrak{A}_{2}$, where $\left|\mathfrak{A}_{i}\right|=3$ and $\left|\boldsymbol{C}_{\mathfrak{z} / \mathbb{x}^{\prime}}\left(\mathfrak{H}_{i}\right)\right|=4, i=1,2$. This implies that $\mathfrak{A}_{1}$ and $\mathfrak{A}_{2}$ both centralize nonidentity elements of $3=\Omega_{1}(\mathfrak{T})$, so $\mathfrak{A}$ centralizes 3, against $i(\mathbb{S})=1$.

Let $\mathfrak{Q}=\boldsymbol{N}_{\mathfrak{\Theta}}(\mathfrak{T}) / \boldsymbol{O}_{2^{\prime}}\left(\boldsymbol{N}_{\mathfrak{\Theta}}(\mathfrak{T})\right)$. Thus $|\mathfrak{Q}|=2^{6} \cdot 3$, and $\mathfrak{Z}$ is a Frobenius group. Let $J$ be an involution of $\mathfrak{I}$. Then $\boldsymbol{C}_{\mathfrak{s}}(J)$ has a normal 2complement, since the 2-length of $\boldsymbol{C}_{\mathscr{\Theta}}(J)$ is one and $\mathbb{Z}$ is a Frobenius group. Suppose $T, T^{\prime} \in \mathfrak{I}$ and $T_{\Im} T^{\prime}$. If $T$ is an involution, we get $T_{\widetilde{N(\mathbb{X})}} T^{\prime}$. Suppose $T$ has order 4 . We may choose $N$ in $N(\mathfrak{I})$ such that $T^{2}=\left(T^{\prime N}\right)^{2}$. Let $T_{1}=T_{1}^{\prime N}$. Then $T$ and $T_{1}$ are conjugate in $\boldsymbol{C}_{\circledast}\left(T^{2}\right)$. As $\boldsymbol{C}_{G}\left(T^{2}\right)$ has a normal 2-complement, $T$ and $T_{1}$ are conjugate in $\mathfrak{T}$. We conclude that elements of $\mathfrak{I}$ are $\mathbb{B S}$-conjugate only if they are $N(\mathfrak{I})$-conjugate. By a result of Glauberman [18], (B) is not simple.

Suppose (d) holds.

Since $i(\mathbb{S})=1$, it follows that $\boldsymbol{C}_{\mathbb{\Theta}}(3) / \boldsymbol{O}_{2^{\prime}}\left(\boldsymbol{C}_{\mathbb{\Theta}}(3)\right)$ is isomorphic to 
the centralizer of a involution of $U_{3}(3)$. By a result of Fong [14], (S) $\cong U_{3}(3)$.

12. The case $2 \in \pi_{3}$. All results in this section are proved on the hypothesis that $2 \in \pi_{3}$. Let $\mathfrak{I}$ be a $S_{2}$-subgroup of $\mathbb{E}$.

LEMma 12.1. Suppose $q \in \pi_{3} \cup \pi_{4}-\{2\}$ and $\mathfrak{\Omega}_{1}$ is a maximal element of $И(\mathfrak{T} ; q)$. Then one of the following holds:

(a) $\left|\mathfrak{D}_{1}\right| \leqq q$.

(b) $\quad N\left(\bigcap_{1}\right)$ contains a $S_{q}$-subgroup of (\$).

(c) $q=3$ and $C_{\mathfrak{x}}\left(\mathfrak{Q}_{1}\right)=1$.

Proof. We may assume that $\left|\mathfrak{\Omega}_{1}\right|>q$ and $\left|N\left(\mathfrak{\Omega}_{1}\right)\right|_{q}<\left|\mathbb{S}_{q}\right|_{q}$. Let $\mathfrak{\Omega}^{*}$ be a $S_{q}$-subgroup of $N\left(\mathfrak{\Omega}_{1}\right)$ permutable with $\mathfrak{T}$ and let $\mathfrak{Q}$ be a $S_{2}$-subgroup of (S) which contains $\mathfrak{Q}^{*}$. Let $\mathfrak{I}_{1}=\boldsymbol{O}_{2}\left(\mathfrak{T} \mathfrak{D}^{*}\right)$.

First, assume that $\mathfrak{I}_{1} \neq 1$.

By Lemma 6.6(i), it follows that $\mathfrak{Z}^{*}$ is a $S_{q}$-subgroup of every solvable subgroup of $(5)$ which contains $\mathfrak{T}_{1} \mathfrak{\Omega}^{*}$.

Let $\mathfrak{A} \in \mathscr{S C C}_{\mathfrak{B}}(\mathfrak{\Omega}), \mathfrak{A}^{*}=\mathfrak{A} \cap \mathfrak{\Omega}^{*}, \mathfrak{A}_{1}=N_{\mathfrak{r}}\left(\mathfrak{Z}^{*}\right)$. By Theorem 6.1, $\mathfrak{A}^{*} \subset \mathfrak{A}$, so $\mathfrak{A}^{*} \subset \mathfrak{A}_{1}$. By Lemma 6.6(i), we conclude that $\mathfrak{H}^{*} \cap \mathfrak{Q}_{1}=1$ and that $C_{\mathfrak{Q}_{1}}(B)=1$ for all $B$ in $\mathfrak{A}_{1}-\mathfrak{A}$. This implies that $\mathfrak{Q}_{1}$ is elementary.

Choose $A$ in $\mathfrak{R}_{1}-\mathfrak{X}^{*}$ with $A^{q} \in \mathfrak{A}^{*}$. Then $\mathfrak{D}_{1}$ and $\mathfrak{\Omega}_{1}^{A}$ are normal elementary subgroups of $\mathfrak{\Omega}^{*}$. If $X \in \mathfrak{Q}_{1} \cap \mathfrak{\Omega}_{1}^{A}$, then $X=A^{-1} Q_{1} A$ with $Q_{1}$ in $\mathfrak{\Omega}_{1}$, so that $\left[Q_{1}, A\right] \in \mathfrak{Q} * \cap \mathfrak{Q}_{1}=1$ so that $Q_{1}=1$, since $C_{\Omega_{1}}(A)=1$. Hence, $\mathfrak{B}=\left\langle\mathfrak{Q}_{1}, \mathfrak{\Omega}_{1}^{A}\right\rangle=\mathfrak{\Omega}_{1} \times \mathfrak{\Omega}_{1}^{A}$ is a normal elementary subgroup of $\mathfrak{\Omega}^{*}$ normalized by $A$. Enlarge $\mathfrak{B}$ to an element $\mathbb{\xi}$ of $\mathscr{S} \mathscr{C} \mathscr{N}\left(\Omega^{*}\right)$ which is normalized by $A$. Since $\left|\mathfrak{Q}_{1}\right|>q$, and since $\mathfrak{Q}^{*}$ is a $S_{q^{-}}$ subgroup of $\boldsymbol{C}\left(Q_{1}\right)$ for all $Q_{1}$ in $\boldsymbol{Z}\left(\Omega^{*}\right) \cap \mathfrak{\Omega}_{1}^{*}$, it follows that $(q, q$, (5) satisfies Hypothesis 6.1. Let $\mathfrak{I}_{2}=\mathfrak{T}_{1}^{A}$. Since $m(\mathfrak{F}) \geqq 3$, we may choose $E$ in $\xi^{-}$so that $C_{\mathfrak{x}_{i}}(E) \neq 1, i=1,2$. Hence, by Theorem 6.1, there is an element $C$ in $C(\mathfrak{F})$ such that $\mathfrak{I}_{2}=\mathfrak{I}_{1}^{C}$. Hence, $A C^{-1}$ normalizes $\mathfrak{I}_{1}$. But $\mathfrak{Z}^{*}$ is a $S_{q}$-subgroup of $N\left(\mathfrak{T}_{1}\right) \cap N(\mathfrak{F})$, since $\mathfrak{\Omega}^{*}$ is a $S_{q}$-subgroup of $N\left(\mathfrak{T}_{1}\right)$. Now every element of $N\left(\mathfrak{I}_{1}\right) \cap N(\mathfrak{F})$ normalizes $C_{\mathbb{E}}\left(\mathfrak{I}_{1}\right)$, so $\mathfrak{B}$ centralizes $\mathfrak{I}_{1}$. This is clearly impossible since $\mathfrak{B} \triangleleft \mathfrak{\Omega}$.

Suppose $\boldsymbol{O}_{2}\left(\mathfrak{I} \mathfrak{\Omega}^{*}\right)=1$. In this case, $\boldsymbol{C}_{\mathfrak{x}}\left(\mathfrak{\Omega}_{1}\right)=1$, so if $q=3$, (c) holds and we are done. Suppose $q \geqq 5$. By a result of Glauberman [17], $\boldsymbol{Z}\left(\boldsymbol{J}\left(\mathfrak{\Omega}^{*}\right)\right) \triangleleft \mathfrak{T} \mathfrak{\Omega}^{*}$. Since $\mathfrak{T} \mathfrak{\Omega}^{*}$ is a $S_{2, q^{-}}$subgroup of $\boldsymbol{N}\left(\boldsymbol{Z}\left(\boldsymbol{J}\left(\mathfrak{\Omega}^{*}\right)\right)\right)$, we get $\mathfrak{\Omega}=\mathfrak{Q}^{*}$ so that (b) holds.

Lemma 12.2. Suppose $q \in \pi_{2}-\{2\}$ and $\mathfrak{D}_{1}$ is a maximal element of $И(\mathfrak{T} ; q)$. Then one of the following holds:
(a) $\left|\mathfrak{Q}_{1}\right| \leqq q$.
(b) $N\left(\bigcap_{1}\right)$ contains a $S_{q}$-subgroup of (B).
(c) $q=3$ and $\mathfrak{\Omega}_{1}$ is cyclic while $\left|\mathbb{S}: N\left(\mathfrak{Q}_{1}\right)\right|_{3}=3$. 
Proof. We may assume that $\left|\mathfrak{\Omega}_{1}\right|>q$ and that $\left|\boldsymbol{N}\left(\mathfrak{\Omega}_{1}\right)\right|_{q}<|\mathbb{S}|_{q}$. Let $\mathfrak{\Omega}^{*}$ be a $S_{q}$-subgroup of $N\left(\mathfrak{\Omega}_{1}\right)$ permutable with $\mathfrak{I}$ and let $\mathfrak{\Omega}$ be a $S_{q}$-subgroup of $\mathbb{S}$ containing $\mathfrak{\Omega}^{*}$. Let $\mathfrak{T}_{1}=\boldsymbol{O}_{2}\left(\mathfrak{T} \mathfrak{Z}^{*}\right)$.

Since $2 \in \pi_{3}, \mathfrak{I}_{1} \neq 1$. By Lemma 6.6(i), $\Omega^{*}$ is a $S_{q}$-subgroup of every solvable subgroup of $\mathbb{S}$ which contains $\mathfrak{Q}^{*} \mathfrak{I}_{1}$. Hence, $\Omega_{1}(Z(\mathfrak{Q})$ ) is contained in $\mathfrak{Q}^{*}$ and is disjoint from $\mathfrak{Q}_{1}$. Hence, $\mathfrak{\Omega}_{1}$ is cyclic since $\Omega^{*}$ contains no elementary subgroup of order $q^{3}$. Since $\left|\mathfrak{l}_{1}\right|>q$, it follows that $\sigma^{1}\left(\bigcap_{1}\right) \neq 1$. Since $\sigma^{1}(\mathfrak{Q})$ centralizes every normal elementary subgroup of $\Omega$, it follows that $\Omega_{1}\left(\Omega_{1}\right) \times \Omega_{1}\left(\boldsymbol{Z}\left(\Omega_{)}\right)\right.$) is a normal elementary subgroup of $\mathfrak{Q}$ of order $q^{2}$. Since $\mathfrak{Q}$ is not abelian, 0.3.8 implies that $\mathfrak{Q}$ is not metacyclic. If $q \geqq 5$, then by 0.34 , we have $\widetilde{O}^{1}(\mathfrak{Q}) \subseteq Z(\mathfrak{Z})$, against $\mathfrak{Q}^{*} \subset \mathfrak{D}$. Hence, $q=3$ and $\mathfrak{Q}^{*}$ is of index 3 in $\supseteq$.

THEOREM 12.1. There are no solvable subgroups $\mathfrak{M}$ of (B) such that

(a) $\quad N(\mathfrak{T}) \subseteq \mathfrak{M}$.

(b) $\mathfrak{M}$ contains the centralizer of each of its involutions.

Proof. Suppose false, and $\mathfrak{M}$ satisfies (a) and (b). We will show that Hypothesis 10.1 is satisfied. Clearly, Hypothesis 10.1 (i), (ii), (iii) are satisfied.

Suppose $p$ is a prime, $P$ is an element of $\mathfrak{M}$ of order $p, P=I J$ is a product of the involutions $I, J$ of $B$, and $P$ is not a product of two involutions of $\mathfrak{M}$. Let $\mathfrak{P}$ be a $S_{p}$-subgroup of $\mathfrak{M}$ with $P \in \mathfrak{P}$. We assume without loss of generality that $\mathfrak{P}$ is permutable with $\mathfrak{T}$. We must show that $\mathfrak{P}$ is cyclic; assume by way of contradiction that $\mathfrak{P}$ is noncyclic. Since $I \in C^{*}(P)$, it follows that $C^{*}(P) \nsubseteq \mathfrak{M}$. If

$$
C_{\widetilde{\Sigma}}(P) \neq 1,
$$

then since $P=I J$, we also have $P=I^{\prime} J^{\prime}$, with $I^{\prime}, J^{\prime}$ being involutions of $\mathfrak{M}$. Hence, $C_{\mathfrak{x}}(P)=1$.

Case 1. $p \in \pi_{2}$.

Since $i(\mathfrak{M})=1, \mathscr{S} \mathscr{C} \mathscr{N}_{3}(\mathfrak{T}) \neq \varnothing$, and since by Lemma 5.40 , $\mathfrak{M}$ has 2-length 1 , it follows that $\mathfrak{I} \triangleleft \mathfrak{I} \mathfrak{P}$.

Case 1(a). $S_{p}$-subgroups of (5) are abelian.

Since $\mathfrak{P}$ is noncyclic, we can choose $P_{0}$ in $\mathfrak{P}^{*}$ with $C_{\mathfrak{x}}\left(P_{0}\right) \neq 1$. Since $C_{\mathfrak{x}}\left(\mathfrak{\beta}_{0}\right)\langle P\rangle$ is a Frobenius group, it follows that $C_{\mathfrak{x}}\left(P_{0}\right)$ contains a four-group. Hence, $C\left(P_{0}\right) \subseteq \mathfrak{M}$, so $\mathfrak{P}$ is a $S_{p}$-subgroup of $\mathbb{S}$. Since $P=I J$, there is an involution $T$ of $B$ which normalizes $\mathfrak{P}$ and inverts $P$. Since $N\left(\left\langle P_{0}\right\rangle\right) \subseteq M$, it follows that $T$ does not invert $\mathfrak{P}$. Hence, $\mathfrak{P}=\mathfrak{P}_{0} \times \mathfrak{P}_{1}$, where $T$ inverts $\mathfrak{P}_{0}$ and centralizes $\mathfrak{P}_{1}$. Choose $P_{1}$ in 
$\mathfrak{P}_{1}^{\sharp}$, and suppose $T \in \mathfrak{M}^{G}$. Then $C_{\mathfrak{m} G}\left(\mathfrak{P}_{1}\right)$ is even, so $C_{\mathfrak{m} G}\left(\mathfrak{P}_{1}\right)$ contains a four-group. Hence, $\boldsymbol{C}\left(\mathfrak{P}_{1}\right) \subseteq \mathfrak{M}^{G}$. Thus, $\mathfrak{M}^{G}$ contains an element of order $p$ which is inverted by $T$. This is not the case, since $\mathfrak{I} \triangleleft \mathfrak{I} \Re$.

Case 1(b). $\quad S_{p}$-subgroups of (S) are nonabelian.

Let $\mathfrak{P}^{*}=C_{\mathfrak{\beta}}(P)$. Since $\mathfrak{P}^{*}$ is noncyclic, we can choose $P^{*}$ in $\mathfrak{P}^{* *}$ such that $C_{\mathfrak{x}}\left(P^{*}\right)$ contains a four-group, so that $C^{*}\left(P^{*}\right) \subseteq \mathfrak{M}$. If $p \geqq 5$, then every $p$-solvable subgroup of $G$ has $p$-length at most 1 . Hence, in this case $\langle P, I\rangle$ can be enlarged to a subgroup $\tilde{\mathfrak{B}}\langle I\rangle$ with

$$
\tilde{\mathfrak{P}} \triangleleft \tilde{\mathfrak{R}}\langle I\rangle,
$$

where $\tilde{\mathfrak{R}}$ is a $S_{p}$-subgroup of $\mathbb{S}$. Then $C_{\widetilde{\mathfrak{B}}}(I) \neq 1$, since $\tilde{\mathfrak{B}}^{\prime} \neq 1$. If $\widetilde{P} \in \widetilde{P}^{*} \cap \boldsymbol{C}(I)$, then $\boldsymbol{C}(\widetilde{P})$ is contained is some conjugate of $\mathfrak{M}$ and so a $S_{2, p}$-subgroup of $C(\widetilde{P})$ is 2-closed. This is impossible, since $I$ does not centralize $C(\widetilde{P})$. We may assume that $p=3$ and that some 3solvable subgroup of $G$ has 3 -length at least 2 . Since $3 \in \pi_{2}$, every subgroup of $(5)$ of odd order is $3^{\prime}$-closed. Hence, there is a 2,3-subgroup $\subseteq$ of $(S)$ with $l_{3}(\subseteq) \geqq 2$. If $\subseteq$ contains a four-group, then $\subseteq \subseteq$ $\mathfrak{M}^{G}$ for some $G$ in $\mathbb{S}$, against $\mathfrak{I} \triangleleft \mathfrak{I} \mathfrak{R}$. Hence, a $S_{2}$-subgroup of $\mathfrak{S}$ is generalized quaternion group, and we have $\boldsymbol{O}_{2}(\mathfrak{S})=1$. Let $\mathfrak{S}_{0}=\boldsymbol{O}_{3}(\mathfrak{S})$. Since $\mathscr{S} \mathscr{C} \mathscr{N}_{3}\left(\mathfrak{S}_{0}\right)=\varnothing$, and since a $S_{2}$-subgroup of $\subseteq$ is faithful represented on $\mathfrak{S}_{0}$, if follows that a $S_{2}$-subgroup $\mathfrak{S}_{2}$ of $\subseteq$ is a quaternion group. We assume without loss of generality that $\mathfrak{S}_{2} \subseteq \mathfrak{M}$. Let $\widetilde{S}_{3}=C_{\Im_{0}}\left(\mathfrak{S}_{2}^{\prime}\right)$. If $\mathfrak{S}_{3} \neq 1$, then $\boldsymbol{C}\left(\mathfrak{S}_{3}\right) \subseteq \mathfrak{M}$, so that $\mathfrak{S}_{2}^{\prime}$ centralizes $\boldsymbol{C}_{\widetilde{\Im}_{0}}\left(\mathfrak{S}_{3}\right)$, since $\mathfrak{I} \triangleleft \mathfrak{I} \mathfrak{P}$. This forces $\mathfrak{S}_{3}=\mathfrak{S}_{0}$, against $\boldsymbol{O}_{2}(\mathfrak{S})=1$. Hence, $\mathfrak{S}_{3}=$ 1 , so that $\mathfrak{S}_{2}^{\prime}$ inverts $\mathfrak{S}_{0}$ and $\mathfrak{S}_{0}=\mathfrak{S}_{01} \times \mathfrak{S}_{02}$, where $\left|\mathfrak{S}_{01}\right|=\left|\mathfrak{S}_{02}\right|=3^{a}$, $\mathfrak{S}_{0 i}$ being cyclic for $i=1,2$. Since $\mathfrak{S}_{2}^{\prime}$ inverts $\mathfrak{S}_{0}$, it follows that $N_{\Im}\left(\mathfrak{S}_{2}\right)$ is a complement to $\mathfrak{S}_{0}$ in $\mathfrak{S}_{\text {. L }} \mathfrak{A}$ be a $S_{3}$-subgroup of $N_{\Im}\left(\mathfrak{S}_{2}\right)$. Since $\mathfrak{U}$ centralizes $\mathfrak{S}_{2}^{\prime}$, we have $\mathfrak{U} \subset \mathfrak{M}$, so $N(\mathfrak{U}) \subseteq \mathfrak{M}$. Hence,

$$
N_{\Im_{0}}(\mathfrak{H}) \subseteq \mathfrak{M}, \quad \text { so } \quad\left[N_{\Xi_{\jmath}}(\mathfrak{U}), \mathfrak{S}_{2}^{\prime}\right] \subseteq O_{3^{\prime}}(\mathfrak{S}) \cap \mathfrak{S}_{0}=1,
$$

against the fact that $\mathfrak{S}_{2}^{\prime}$ inverts $\mathfrak{S}_{0}$.

Case 2. $p \in \pi_{3} \cup \pi_{4}$.

Let $\mathfrak{P}^{*}$ be a $S_{p}$-subgroup of $\mathbb{B}$ which contains $\mathfrak{P}$.

Case 2(a). $\mathfrak{P} \subset \mathfrak{P}^{*}$.

Since $\mathfrak{P}$ is noncyclic, Theorem 10.9 implies that

$$
\mathfrak{P} \notin \mathscr{A}_{4}\left(\mathfrak{P}^{*}\right) \text {. }
$$

Hence, $\mathfrak{P}$ contains a cyclic subgroup $\mathfrak{P}_{0}$ of index $p$. Also, $\mathfrak{T} \triangleleft \mathfrak{T} \mathfrak{P}$. If $P \notin Z(\mathfrak{Z})$, then $\mathfrak{P}=\mathfrak{P}_{0}\langle P\rangle$ and $\Omega_{1}\left(\mathfrak{P}_{0}\right)$ centralizes $\mathfrak{T}$. In this case, $\Omega_{1}\left(\mathfrak{P}_{0}\right) \subseteq \boldsymbol{Z}\left(\mathfrak{S}^{*}\right)$, so $\mathfrak{P}=\mathfrak{S}^{*}$ against our hypothesis. Hence, $P \in \boldsymbol{Z}(\mathfrak{P})$. 
If $\mathfrak{P}$ is nonabelian, then the normalizer of every noncentral subgroup of $\mathfrak{P}$ of order $p$ is contained in $M$. This is clearly impossible, since $\mathfrak{P}^{\prime} \neq 1$ implies that $C_{\Re^{*}}(\mathfrak{U}) \nsubseteq \mathfrak{P}$ for all noncentral subgroups $\mathfrak{U}$ of $\mathfrak{P}$ of order $p$. Hence, $\mathfrak{P}=\mathfrak{P}_{0} \times \mathfrak{P}_{1}$ is abelian, and exactly one subgroup of $\mathfrak{B}$ of order $p$ has a non trivial fixed point on $\mathfrak{T}$. Hence, if $T$ is an involution of $\mathbb{B}$, then $|\boldsymbol{C}(T)|_{p}=p$. It follows that $P \in \boldsymbol{Z}\left(\mathfrak{P}^{*}\right)$ and $\mathfrak{P}^{*}$ is normalized by some involution $T$ of $\mathbb{B}$. Clearly, $\mathfrak{P}^{* \prime} \neq 1$, since $\mathfrak{P} \subset \mathfrak{P}^{*}$. If $P^{*} \in \boldsymbol{C}_{\mathfrak{F}^{*}}(T)^{\sharp}$, then $\boldsymbol{C}\left(P^{*}\right)$ contains $\left\langle T, Z\left(\mathfrak{P}^{*}\right)\right\rangle$ and $C\left(P^{*}\right)$ is contained in some conjugate of $M$. This is impossible, since $T$ inverts $\boldsymbol{Z}\left(\mathfrak{P}^{*}\right)$ and $\mathfrak{I} \triangleleft \mathfrak{I} \mathfrak{P}$.

Case $2(\mathrm{~b}) . \quad \mathfrak{P}^{3}=\mathfrak{P}^{*}$.

Since $C^{*}(P) \nsubseteq \mathfrak{M}$, it follows that a $S_{\mathfrak{\beta}}$-subgroup of $C_{\mathfrak{n}}(P)$ is of the shape $\langle P\rangle \times \sqrt{5}$, where $\sqrt{5}$ is cyclic. By Theorem $10.9,\langle P\rangle \times \sqrt{5}$ is a $S_{p}$-subgroup of $C(P)$, so there is an involution of $G$ which normalizes $\langle P\rangle \times \mathfrak{C}$ and inverts $P$. But $N(\langle P\rangle \times C) \subseteq \mathfrak{M}$, by Theorem 10.9, and so $P$ is a product of two involutions of $\mathfrak{M}$, against our hypothesis. We have verified Hypothesis 10.1(iv).

In verifying Hypothesis 10.1(v), we may assume that $P$ is of prime order $p$. Let $\mathfrak{P}$ be a $S_{p}$-subgroup of $\boldsymbol{O}_{2^{\prime}}(\mathfrak{M})$ which contains $P$. We assume without loss of generality that $\mathfrak{I}$ normalizes $\mathfrak{P}$. If $\boldsymbol{C}_{\mathfrak{P}}(P)$ contains no elementary subgroup of order $p^{3}$, then since $i(\mathfrak{M})=1$, it follows that $\mathfrak{I}$ centralizes $P$, so that $C^{*}(P) \subseteq \mathfrak{M}$ and (v) holds. If $\boldsymbol{C}_{\Re}(P)$ contains an elementary subgroup of order $p^{3}$, then once again $C^{*}(P) \subseteq \mathfrak{M}$, by Theorem 10.2. The proof is complete.

Theorem 10.2 and Theorem 12.1 imply that $\langle\mathfrak{F} \mid \mathfrak{S} \in \boldsymbol{U}(\mathfrak{T})\rangle$ is nonsolvable. We will frequently use this fact.

THEOREm 12.2. Assume the following:

(i) $p, q \in \pi_{3}-\{2,3\}, \pi=\{p, q\}, r \in \pi^{\prime}$.

(ii) $\mathfrak{S}$ is a $S_{p, q}$-subgroup of $\left(\mathbb{S} ; \mathfrak{P}\right.$ is a $S_{p}$-subgroup of $\mathfrak{S} ; \mathfrak{Q}$ is a $S_{q}$-subgroup of $\mathfrak{S}$.

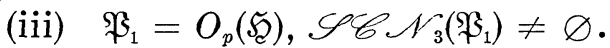

(iv) $\mathfrak{\Omega}_{1}=O_{q}(\mathfrak{S}), \mathscr{S P C}_{\mathfrak{C}} \mathscr{N}_{3}\left(\mathfrak{\Omega}_{1}\right) \neq \varnothing$.

(v) $\mathfrak{A} \in \mathscr{S} \mathscr{C} \mathscr{N}_{3}\left(\mathfrak{P}_{1}\right), \mathfrak{A} \triangleleft \mathfrak{P}, \mathfrak{A} * \in \mathscr{S} \mathscr{C} \mathscr{N}(\mathfrak{P}), \mathfrak{A} \leqq \mathfrak{A *}$.

(vi) $\mathfrak{B} \in \mathscr{S P C}_{\mathfrak{N}}\left(\mathfrak{D}_{1}\right), \mathfrak{B} \triangleleft \mathfrak{\Omega}, \mathfrak{B}^{*} \in \operatorname{SPC} \mathscr{N}(\mathfrak{\Omega}), \mathfrak{B} \subseteq \mathfrak{B}^{*}$.

Then the following hold.

(i) $C\left(\mathfrak{P}_{1} \mathfrak{B}\right)$ permutes transitively by conjugation the maximal elements of $И\left(\Re_{1} \mathfrak{B} ; r\right)$.

(ii) Suppose $\mathfrak{B}$ is faithfully represented on some element of $И(\mathfrak{B} ; r)$. Then there is an element $\mathfrak{R}$ in $И\left(\mathfrak{P}_{1} \mathfrak{\Omega}_{1} ; r\right)$ with the following properties:

(a) $\mathfrak{S}$ normalizes $\Re$.

(b) $\mathfrak{B}$ is faithfully represented on $\mathfrak{R}$. 
Proof. (i) Hypothesis 6.5 is satisfied with $\mathfrak{P}_{1}$ in the role of $\AA$, $\mathfrak{\Omega}_{1}$ in the role of $\mathfrak{\Omega}$. By Lemma 6.5, we conclude that $\mathfrak{P}_{1}$ centralizes every element of $\boldsymbol{U}\left(\mathfrak{P}_{1} ; q\right)$. We must show that $\mathfrak{B}$ centralizes every element of $\boldsymbol{U}(\mathfrak{B} ; p)$. Choose $\mathfrak{B}_{1} \in \mathscr{U}^{*}(\mathfrak{D}), \mathfrak{B}_{1} \subseteq \mathfrak{B}$. By Lemma 6.4 (with $p$ and $q$ interchanged), $\mathfrak{B}_{1}$ centralizes every element of $U\left(\mathfrak{B}_{1} ; p\right)$. Suppose $\mathfrak{F} \in \boldsymbol{U}(\mathfrak{B} ; p)$. Choose $\boldsymbol{Z}$ in $\mathfrak{B}_{1}^{*} \cap \boldsymbol{Z}(\mathfrak{\Omega})$. Then $\left\langle\mathfrak{P}_{1}, \mathfrak{F}, \mathfrak{l}\right\rangle \subseteq \boldsymbol{C}(\boldsymbol{Z})$. Since $\mathfrak{P}_{1}$ is a maximal element of $U(\mathfrak{Q} ; p)$, it follows that $\mathfrak{P}_{1}$ is a $S_{p}$-subgroup of $\boldsymbol{O}_{q^{\prime}}(\boldsymbol{C}(Z))$. Since $q \geqq 5, \mathfrak{B} \subseteq \boldsymbol{O}_{q^{\prime}}{ }_{q}(\boldsymbol{C}(3))$. Hence,

$$
[\mathfrak{F}, \mathfrak{B}] \subseteq \boldsymbol{O}_{q^{\prime}}(C(Z)) \text {. }
$$

Since $\mathfrak{B}$ centralizes $\mathfrak{B}_{1}, \mathfrak{B}$ centralizes every p-subgroup of $\boldsymbol{O}_{q^{\prime}}(\boldsymbol{C}(Z)$ ) which $\mathfrak{B}$ normalizes. Hence, $[\mathfrak{F}, \mathfrak{B}, \mathfrak{B}]=1$, so $\mathfrak{B}$ centralizes $\mathfrak{F}$.

We next show that $\boldsymbol{C}\left(\mathfrak{P}_{1} \mathfrak{B}\right)=\boldsymbol{Z}\left(\mathfrak{P}_{1} \mathfrak{B}\right) \times \mathfrak{\Im}$ where $\mathfrak{\Im}$ is a $\pi^{\prime}$-group. Since $C\left(\mathfrak{P}_{1} \mathfrak{B}\right) \subseteq C\left(\mathfrak{P}_{1}\right) \subseteq N\left(\mathfrak{P}_{1}\right)$, and since $\mathfrak{S} \subseteq N\left(\mathfrak{P}_{1}\right)$, the desired equality follows from Lemma 5.19.

Let $\mathscr{F}=\left\{\mathfrak{F}_{1}, \mathfrak{B}\right\}$. We will show that Hypothesis 6.2 is satisfied with $\mathfrak{P}_{1} \mathfrak{B}$ in the role of $\mathfrak{A}, r$ in the role of $q$. (a), (b), (c) have been verified, and (d)(ii) holds by construction. Since $\mathfrak{S}_{\mathfrak{C}} \subseteq N\left(\mathfrak{P}_{1}\right)$, (d)(i) holds

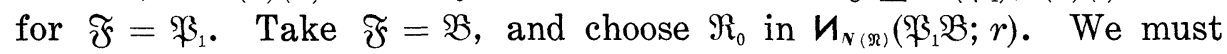
show that $\Re_{0} \subseteq \boldsymbol{O}_{\pi^{\prime}}(\boldsymbol{N}(\mathfrak{B}))$. Let $\mathfrak{S}^{*}$ be a $S_{p, q}$-subgroup of $\boldsymbol{N}(\mathfrak{B})$ which contains $\mathfrak{Q}_{1}$. Since, $\mathfrak{P}_{1}$ is a maximal element of $U(\Omega ; p)$, it follows that $\mathfrak{P}_{1}=\boldsymbol{O}_{p}\left(\mathfrak{S}^{*}\right)$. Hence, Theorem 2 of [41] applies to yield (d)(i). By construction, (e)(i) holds.

Since $\mathbb{B S}^{3}$ is an $N$-group with $2 \in \pi^{\prime}$, it follows that $\left(\pi, r, \mathfrak{P}_{1} \mathfrak{B}\right)$ satisfies conditions (b), (d), (e) of Hypothesis 6.1. By Theorem 6.2, $\left(\pi, r, \mathfrak{P}_{1} \mathfrak{B}\right)$ satisfies Hypothesis 6.1 . Since $m(\mathfrak{B}) \geqq 3$, Theorem 6.1 yields (i).

We remark that by symmetry we get also

(i $)^{\prime} \quad C\left(\mathfrak{2} \mathfrak{\Omega}_{1}\right)$ permutes transitively by conjugation the maximal elements of $И\left(\mathfrak{2} \Omega_{1} ; r\right)$.

It remains to verify (ii). Choose $B$ in $\mathfrak{B}^{*}$ and let $\Re_{0}$ be an element of $U\left(\mathfrak{B}^{*} ; r\right)$ which is not centralized by $B$. Choose $\mathfrak{B}_{1} \in \mathscr{C}^{*}(\mathfrak{Q})$ with $\mathfrak{B}_{1} \subset \mathfrak{B}$. Then choose $B_{1}$ in $\mathfrak{B}_{1}^{\sharp}$ so that $B$ does not centralize

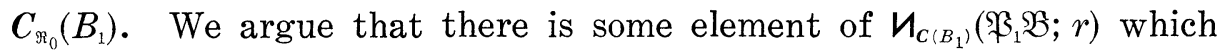
is not centralized by $B$. In any case, $\left[C_{\Re_{0}}\left(B_{1}\right), B\right]$ lies in $\boldsymbol{O}_{q^{\prime}}\left(C\left(B_{1}\right)\right)$,

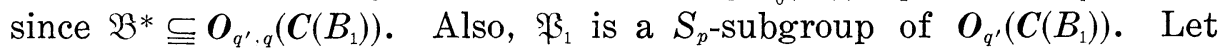
$\Re_{1}$ be a $S_{r}$-subgroup of $\boldsymbol{O}_{q^{\prime}}\left(\boldsymbol{C}\left(B_{1}\right)\right)$ which is normalized by $\mathfrak{B}^{*}$ and is permutable with $\mathfrak{P}_{1}$. Then $B$ does not centralize $\Re_{1}$. Thus, $B$ does not centralize the Fitting subgroup of $\mathfrak{P}_{1} \Re_{1}$. Since $B$ centralizes $\mathfrak{P}_{1}$, $B$ does not centralize the $S_{r}$-subgroup of the Fitting subgroup of $\mathfrak{P}_{1} \Re_{1}$, and our assertion is established.

Let $\mathfrak{R}$ be a maximal element of $И\left(\mathfrak{P}_{1} \mathfrak{B} ; r\right)$ which is not centralized by $\mathfrak{B}$. We will show that $N(\Re)$ contains a $S_{q}$-subgroup of $N\left(\mathfrak{P}_{1} \mathfrak{B}\right)$. 
Choose $N$ in $N\left(\mathfrak{P}_{1} \mathfrak{B}\right)$. Then $\Re^{N}$ is a maximal element of $U\left(\mathfrak{P}_{1} \mathfrak{B} ; r\right)$, so $\mathfrak{R}^{N}=\mathfrak{R}^{C}$ for some $C$ in $C\left(\mathfrak{P}_{1} \mathfrak{B}\right)$. Hence,

$$
N\left(\mathfrak{P}_{1} \mathfrak{B}\right)=\left(N\left(\mathfrak{P}_{1} \mathfrak{B}\right) \cap N(\mathfrak{R})\right) C\left(\mathfrak{P}_{1} \mathfrak{R}\right) .
$$

Since $\boldsymbol{C}\left(\mathfrak{P}_{1} \mathfrak{B}\right)=\boldsymbol{Z}\left(\mathfrak{B}_{1} \mathfrak{B}\right) \times \mathfrak{F}$, where $\mathfrak{\Im}$ is a $\pi^{\prime}$-group, our assertion follows. Let $\mathfrak{S}^{*}$ be a $S_{p q}$-subgroup of $N\left(\mathfrak{P}_{1} \mathfrak{B}\right) \cap N(\mathfrak{R})$.

We have shown that if $B \in \mathfrak{B}^{\sharp}$, then there is a maximal element $\mathfrak{R}=\mathfrak{R}(B)$ of $И\left(\mathfrak{P}_{1} \mathfrak{B} ; r\right)$ which is not centralized by $B$. By (i), we conclude that $\mathfrak{B}$ is faithfully represented on every maximal element of $И\left(\mathfrak{P}_{1} \mathfrak{B} ; r\right)$.

Since $\mathfrak{Q} \subseteq N\left(\mathfrak{P}_{1} \mathfrak{B}\right)$, we can choose $N$ in $N\left(\mathfrak{P}_{1} \mathfrak{B}\right)$ so that $\mathfrak{K}^{* N} \supset \mathfrak{D}$. Replacing $\Re$ by $\Re^{N}$ we may assume that $\Re$ is a maximal element of $\mathcal{U}\left(\mathfrak{P}_{1} \mathfrak{B} ; r\right)$ which is normalized by $\mathfrak{P}_{1} \mathfrak{Q}$ and on which $\mathfrak{B}$ is faithfully represented. Since $\Re$ is a maximal element of $U\left(\mathfrak{B}_{1} \mathfrak{B} ; r\right)$ normalized by $\mathfrak{P}_{1} \mathfrak{Q}_{1}, \mathfrak{R}$ is a maximal element of $И\left(\mathfrak{P}_{1} \mathfrak{D}_{1} ; r\right)$. However, we argue that $\Re$ is also a maximal element of $U\left(\mathfrak{X} \Omega_{1} ; r\right)$. Let $\Re^{*}=N(\Re)$. By (i) applied to $\mathfrak{Y \Omega}_{1}$, we see that $\left(\pi, r, \mathfrak{M} \mathfrak{\Omega}_{1}\right)$ satisfies Hypothesis 6.1. Hence, every element of $\boldsymbol{U}_{\Re^{*}}\left(\mathfrak{L}_{1} ; r\right)$ lies in $\boldsymbol{O}_{\pi^{\prime}}\left(\mathfrak{R}^{*}\right)$. As $\Re$ is a maximal

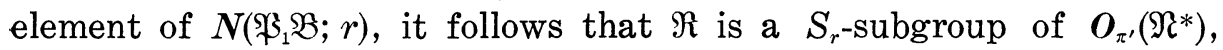
so is a maximal element of $\boldsymbol{U}\left(\mathfrak{H O}_{1} ; r\right)$.

By (i) applied to $\mathfrak{X}_{1}$, it follows that $N(\Re)$ contains a $S_{p}$-subgroup of $N\left(\mathfrak{R}_{1}\right)$. Let $\mathfrak{S}^{*}$ be a $S_{p}$-subgroup of $N(\Re)$ permutable with $\cong$, such that $\mathfrak{P}_{1} \subseteq \mathfrak{P}^{*}$. Then $\mathfrak{P}_{1}=\boldsymbol{O}_{p}\left(\mathfrak{P}^{*} \mathfrak{Q}\right)$, so $\left\langle\mathfrak{P}, \mathfrak{P}^{*}, \mathfrak{Q}\right\rangle \subseteq N\left(\mathfrak{P}_{1}\right)$. Now we have the exploitable equality $\mathfrak{\Omega}_{1}=\boldsymbol{O}_{p^{\prime}}\left(N\left(\mathfrak{P}_{1}\right)\right) \cap \mathfrak{D}$. We choose $N_{1}$ in $N\left(\mathfrak{P}_{1}\right)$ so that $\mathfrak{P}^{*^{N_{1}}}=\mathfrak{P}, \mathfrak{Q}^{N_{1}}=\mathfrak{\Omega}$. The expression for $\mathfrak{Q}_{1}$ guarantees that $N_{1} \in N\left(\mathfrak{\Omega}_{1}\right)$. Thus, $\mathfrak{R} \mathfrak{Q}$ normalizes $\Re^{N_{1}}$ and since $\Re^{N_{1}}$ is a maximal element of $U\left(\mathfrak{B}_{1} \mathfrak{B} ; r\right), \mathfrak{B}$ is faithfully represented on $\mathfrak{R}^{N_{1}}$; the proof is complete.

HYPoTHeSIS 12.1. (i ) $p \in \pi_{4}, p \geqq 5$.

(ii) (5) satisfies $E_{2 p}$.

We will show that Hypothesis 12.1 is not satisfied. Suppose false. Let $\mathfrak{B}$ be a $S_{p}$-subgroup of $(S)$ permutable with $\mathfrak{I}$, and let $\mathfrak{M}=M(\mathfrak{P})$, $\mathfrak{M}$ being available by Theorem 10.2

Let $\mathfrak{P}_{0}=\boldsymbol{O}_{p}(\mathfrak{P} \mathfrak{T})$ and let $\mathfrak{Q}$ be a maximal element of $И(\mathfrak{T} ; q), q$ being an odd prime. We will show that $\mathfrak{M} \mathfrak{M}$. First, suppose that $\mathfrak{I}$ contains an elementary subgroup of order 16 . In this case, $\mathfrak{I}$ contains a four-subgroup $\mathbb{F}$ such that $\boldsymbol{C}_{\mathfrak{P}_{0}}(\mathfrak{F}) \in \mathscr{A}_{4}(p)$. Hence, $\boldsymbol{C}(E) \subseteq \mathfrak{M}$ for all $E$ in $\mathfrak{F}^{\sharp}$, by Theorem 10.2 , so $\mathfrak{Q} \subseteq \mathfrak{M}$. We may therefore assume that $\mathfrak{I}$ contains no elementary subgroup of order 16 . Suppose that $\mathfrak{M} \mathbb{M}$.

Let $\mathbb{5}$ be a normal elementary subgroup of $\mathfrak{I}$ of order 8 . Let 
$\mathfrak{Q}_{0}=\mathfrak{Q} \cap \mathfrak{M}, \mathfrak{\Omega}_{1} \subseteq \mathfrak{\Omega},\left|\mathfrak{Q}_{1}: \mathfrak{\Omega}_{0}\right|=q$, with $\left(5 \subseteq N\left(\mathfrak{\Omega}_{1}\right) . \quad\right.$ Let

$$
\mathfrak{F}_{0}=C_{\varpi}\left(\mathfrak{\Omega}_{1} / \mathfrak{\Omega}_{0}\right) \text {. }
$$

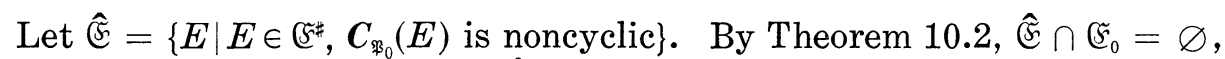
so $E E^{\prime} \in \mathfrak{F}_{0}$ for all $E, E^{\prime}$ in $\hat{\mathfrak{F}}$. By Lemma 5.39 with $\mathfrak{P}_{0}$ in the role of $\mathfrak{P}$, it follows that $\mathfrak{I}$ is isomorphic to a subgroup of $G L(3, p)$. Since $\mathfrak{M}$ dominates $\mathfrak{I}$, it follows that $\mathfrak{I}^{\prime} \neq 1$. However, this violates Lemma $5.43(\mathrm{~b})$.

Since $\mathfrak{Q} \subseteq \mathfrak{M}, \mathfrak{M}$ contains every element of $И(\mathfrak{T})$. Hence,

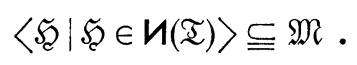

This is not the case since $\mathfrak{M}$ is solvable.

HYPOTHESIS 12.2. ( i ) $p \in \pi_{3}, p \geqq 5$.

(ii) Maximal elements of $И(\mathfrak{T} ; p)$ contain elementary subgroups of order $p^{3}$.

We will show that Hypothesis 12.2 is not satisfied. Suppose false.

Let $\mathfrak{P}$ be a $S_{p}$-subgroup of $\mathbb{S}$ permutable with $\mathfrak{I}$. $\mathfrak{B}$ is available by Lemma 12.1. Let $\sigma$ be the equivalence class of $\pi_{3}-\{2\}$ under $\sim$ which contains $p$, and let $\pi=\sigma \cup \tau(\sigma)$. Let $\Re$ be a $S_{\pi}$-subgroup of (S) which contains $\mathfrak{P}$. Let $\mathfrak{P}_{0}=\boldsymbol{O}_{p}(\mathfrak{P} \mathfrak{I}), \mathfrak{I}_{0}=\boldsymbol{O}_{2}(\mathfrak{P} \mathfrak{I})$. Thus, by Lemma

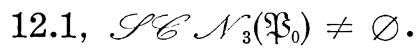

Suppose $q \in \pi, q \neq p$ and $\Omega$ is a $S_{q}$-subgroup of $\mathfrak{R}$ permutable with $\mathfrak{P}$. Let $\mathfrak{\Omega}_{1}=\boldsymbol{O}_{q}(\mathfrak{P} \mathfrak{Q}), \mathfrak{P}_{1}=\boldsymbol{O}_{p}(\mathfrak{P} \mathfrak{Q})$. We will show that

(*) $\quad\left[\mathfrak{P}_{0}, \mathfrak{\Omega}_{1}\right]$ centralizes every element of $\boldsymbol{U}\left(\Omega_{1} ; 2\right)$.

Suppose $(*)$ does not hold.

Since $(*)$ does not hold, it follows readily that (8) does not satisfy $E_{2, p q}^{s}$. We will exploit this fact.

If $\mathfrak{I}_{0}=1$, then by Theorem $6.1, N\left(\mathfrak{\Omega}_{1}\right)$ contains a $\mathfrak{S}_{2 p}$-subgroup $^{\text {-subgen }}$ of (S). We may assume therefore that

$$
\mathfrak{T}_{0} \neq 1 \text {. }
$$

Suppose $\mathfrak{P}_{0} \cap \mathfrak{P}_{1}=\mathfrak{\Im} \neq 1$. Let $\&$ be a $S_{2, p}$-subgroup of $N(\mathfrak{\Im})$ with $\mathfrak{P} \subset \mathbb{R}$. We assume without loss of generality that $\mathfrak{I}_{0} \mathfrak{Q}_{1}=\boldsymbol{O}_{p}(\mathfrak{R})$.

Let $\tilde{\mathfrak{P}}=\mathfrak{P} \cap \boldsymbol{O}_{2}(\mathfrak{P} \mathfrak{T}), \quad x \in \boldsymbol{N}(\mathfrak{P}) . \quad$ By $\quad$ Theorem 6.1, $\quad\left(\mathfrak{I}_{0} \mathfrak{Q}_{1}\right)^{x} \subseteq$ $\left\langle\mathfrak{I}_{0} \mathfrak{Q}_{1}, C(\tilde{\mathfrak{P}})\right\rangle \subseteq C(\mathfrak{I}) \subseteq N(\mathfrak{I})$, and so $\left(\mathfrak{I}_{0} \mathfrak{I}_{1}\right)^{x}=\left(\mathfrak{I}_{0} \mathfrak{I}_{1}\right)^{c}$ for some $c \in C(\underset{\mathfrak{T}}{\tilde{\mathfrak{S}}})$. This implies that $N\left(\mathfrak{T}_{0} \mathfrak{Q}_{1}\right)$ contains a $S_{2}$-subgroup of $\mathbb{\widetilde { S }}$. Let $\quad \widetilde{\mathfrak{P}}=\mathfrak{P} \cap \boldsymbol{O}_{q, p}(\mathfrak{P} \mathfrak{Q}), \quad y \in N(\widetilde{\widetilde{\mathfrak{P}}})$. Then $\left(\mathfrak{I}_{0} \mathfrak{\Omega}_{1}\right)^{y} \cong\left\langle\mathfrak{I}_{0} \mathfrak{\Omega}_{1}, \boldsymbol{C}(\widetilde{\widetilde{\mathfrak{F}}})\right\rangle \leqq$ $C(\mathfrak{\Im}) \subseteq N(\mathfrak{\Im})$, so $\left(\mathfrak{I}_{0} \mathfrak{Q}_{1}\right)^{y}=\left(\mathfrak{I}_{0} \mathfrak{\Omega}_{1}\right)^{d}$ for some $d \in C(\widetilde{\mathfrak{F}})$. Hence, $N\left(\mathfrak{I}_{0} \mathfrak{\Omega}_{1}\right)$ contains a $S_{q}$-subgroup of $\mathbb{S}$, and so $N\left(\mathfrak{I}_{0} \mathfrak{Q}_{1}\right)$ contains a $S_{2 p q}$-subgroup of (8). This is false, and so 


$$
\mathfrak{P}_{0} \cap \mathfrak{P}_{1}=1 \text {. }
$$

Since $\mathscr{S} \mathscr{C} \mathscr{N}_{3}\left(\mathfrak{P}_{0}\right) \neq \varnothing,(12.2)$ implies that

$$
\mathscr{S} \mathscr{C} \mathscr{N}_{3}\left(\mathfrak{\Omega}_{1}\right) \neq \varnothing \text {. }
$$

Let $\left[\mathfrak{P}_{0}, \mathfrak{\Omega}_{1}\right]=\mathfrak{Q}_{2}$. Since $(*)$ is false, some element $\mathfrak{D}$ of $N\left(\mathfrak{\Omega}_{1} ; 2\right)$ is not centralized by $\mathfrak{\Omega}_{2}$. Suppose $\mathfrak{\Omega}_{2}$ centralizes every element of $И(\mathfrak{Q} ; 2)$. Note that $\mathfrak{P}$ normalizes $\mathfrak{\Omega}_{2}$. Let $\widetilde{\mathfrak{Q}}_{2}=\mathfrak{\Omega}_{2}^{\mathfrak{Q}}=\mathfrak{\Omega}_{2}^{\mathfrak{R a}}$, so that $\tilde{\mathfrak{Q}}_{2}$ centralizes every element of $U(\mathfrak{\Omega} ; 2)$. Since $\mathfrak{B} \subseteq N\left(\mathfrak{\Omega}_{1}\right)^{\prime}$, it follows that $\mathfrak{\Omega}_{2}$ is not cyclic. Thus, $\tilde{\mathfrak{D}}_{2}$ contains a subgroup $\mathfrak{B}$ of type $(q, q)$ with $\mathfrak{B} \triangleleft \mathfrak{Q}$, and it follows that $\mathfrak{B}$ centralizes every element of $И(\mathfrak{\Omega} ; 2)$, whence $\mathfrak{B}$ centralizes every element of $\boldsymbol{U}(\mathfrak{B} ; 2)$. Let $\widetilde{\mathfrak{B}}=\mathfrak{B}^{\mathfrak{P}}=\mathfrak{B}^{\mathfrak{R}}$, so that $\widetilde{\mathfrak{B}} \cong \widetilde{\mathfrak{D}}_{2}$, and $\widetilde{\mathfrak{B}}$ centralizes every element of $И(\widetilde{\mathfrak{B}} ; 2)$. Since $\widetilde{\mathfrak{D}}_{2} \subseteq \mathfrak{Q}_{1}$, we have $\widetilde{\mathfrak{B}} \subseteq \mathfrak{D}_{1}$, and $\mathfrak{D} \in \mathcal{H}(\mathfrak{\mathfrak { B }} ; 2)$. Thus, $\langle\mathfrak{D}, \mathfrak{P}, \mathfrak{D}\rangle \subseteq$ $\boldsymbol{N}(\widetilde{\mathfrak{B}})$, and $\mathfrak{D}_{1}$ is a $S_{q}$-subgroup of $\boldsymbol{O}_{p^{\prime}}(\boldsymbol{N}(\mathfrak{B}))$. Now $\mathfrak{D}_{1}=\left[\mathfrak{D}, \mathfrak{Q}_{1}\right] \supseteqq$ $\left[\mathfrak{D}, \mathfrak{\Omega}_{2}\right] \neq 1$ and $\mathfrak{D}_{1} \subseteq \boldsymbol{O}_{q^{\prime}}\left(\boldsymbol{O}_{p^{\prime}}(\boldsymbol{N}(\mathfrak{B}))\right)$. This implies that $\mathfrak{\Omega}_{2}$ does not centralize any $S_{2}$-subgroup of $\boldsymbol{O}_{q^{\prime}}\left(\boldsymbol{O}_{p^{\prime}}(\boldsymbol{N}(\mathfrak{B}))\right)$, against our assumption that $\mathfrak{\Omega}_{2}$ centralizes every element of $U(\mathfrak{D} ; 2)$. We conclude that $\left[\mathfrak{P}_{0}, \mathfrak{Z}_{1}\right]$ does not centralize every elememt of $\boldsymbol{U}(\mathfrak{\Omega} ; 2)$. By Theorem $6.1,\left[\mathfrak{P}_{0}, \mathfrak{D}_{1}\right]$ does not centralize any element of $\boldsymbol{U}^{*}(\mathfrak{\Omega} ; 2)$.

Suppose $\mathfrak{P}_{1}=1$. In this case, there is a maximal element $\mathfrak{R}$ of $\Lambda(\cong ; 2)$ which is normalized by $\mathfrak{P}$, so $\left(^{*}\right)$ holds since $\left[\mathfrak{P}_{0}, \Re\right]=1$.

Suppose $\mathfrak{\beta}_{1}$ is a nonidentity cyclic group. Let $\&$ be a normal elementary subgroup of $\mathfrak{P}$ of order $p^{3}$ with $\mathfrak{F} \subseteq \mathfrak{P}_{0}$. Choose a maximal element $\Re$ of $U(\mathfrak{D} ; 2)$ such that $N(\Re) \cap \Re$ is a $S_{p}$-subgroup of $N(\Re)$. Let $\mathfrak{F}^{*}=N(\Re) \cap \mathfrak{P}$, so that $\mathfrak{F}^{*} \mathfrak{Z}$ is a $S_{p}$-subgroup of $N(\Re)$ and $\mathfrak{P}^{*} \mathfrak{P}_{1}=\mathfrak{B}$. Let $\mathfrak{F}^{*}=\mathfrak{F} \cap \mathfrak{P}^{*}$. Since $\mathfrak{F}_{1}$ is cyclic, $\mathfrak{F}^{*}$ is noncyclic. Since $\mathfrak{P}_{1} \subseteq Z(\mathfrak{B})$, 丞 $\triangleleft \mathfrak{P}$. Let $\mathfrak{F}_{1}^{*} \triangleleft \mathfrak{P}, \mathfrak{F}_{1}^{*} \subseteq \mathfrak{F},\left|\mathfrak{F}_{1}^{*}\right|=p^{2}$. Then

$$
\left[\mathfrak{G}_{1}^{*}, \mathfrak{Q}_{1}\right]=\mathfrak{Q}_{1}^{*}
$$

centralizes $\Re$ and $\mathfrak{\Omega}_{1}^{*}$ is normalized by $\mathfrak{P}$. Hence, $N\left(\mathfrak{\Omega}_{1}^{*}\right)$ contains $\mathfrak{\Omega}_{1}, \mathfrak{R}$ and $\mathfrak{P}$. Let $\tilde{\Re}$ be a $S_{p}$-subgroup of $N\left(\mathfrak{\Omega}_{1}^{*}\right)$ which contains $\mathfrak{\Omega}_{1} \mathfrak{P}$. Since $\mathfrak{\Omega}_{1}=\boldsymbol{O}_{q}(\tilde{\Re}),\left(^{*}\right)$ follows immediately.

Suppose $\mathscr{S} \mathscr{C} \mathscr{N}_{3}\left(\mathfrak{P}_{1}\right)=\varnothing$. Proceeding as before, choose a maximal element $\mathfrak{R}$ of $\boldsymbol{U}\left(\mathfrak{\Omega}_{1} ; 2\right)$ such that $N(\mathfrak{R}) \cap \mathfrak{P}=\mathfrak{P}^{*}$ is a $S_{p}$-subgroup of $N(\Re)$. Since $\mathscr{S}_{\mathfrak{C}} \mathscr{N}_{3}\left(\mathfrak{P}_{1}\right)=\varnothing$, it follows that $\mathfrak{P}^{*} / \boldsymbol{C}_{\mathfrak{F}^{*}}\left(\mathfrak{P}_{1}\right)$ is elementary of order $1, p$ or $p^{2}$. If $\mathfrak{P}^{*} \cap \mathfrak{P}_{0}$ is noncyclic, then since $\mathfrak{P}^{*} \cap$ $\mathfrak{P}_{0}$ centralizes $\mathfrak{P}_{1}$, it follows that $\mathfrak{P}^{*} \cap \mathfrak{P}_{0}$ contains an elementary subgroup $\mathfrak{F}$ of order $p^{2}$ which is normal in $\mathfrak{P}$. Since $\mathfrak{F}$ centralizes $\mathfrak{R}$, so does $\left[\mathfrak{l}_{1}, \mathfrak{F}\right]$, so $\left(^{*}\right)$ is seen to hold. We may assume that $\mathfrak{P}^{*} \cap \mathfrak{P}_{0}$ is cyclic. Since $\mathscr{S}^{*} \mathscr{N}_{3}\left(\mathfrak{P}_{1}\right)=\varnothing$, it follows that $\mathfrak{P}^{*} \cap \mathfrak{P}_{0} \neq 1$. Suppose $\mathfrak{P}^{*} \cap \boldsymbol{C}\left(\mathfrak{P}_{1}\right)$ is noncyclic. Since $\Omega_{1}\left(\mathfrak{P}^{*} \cap \mathfrak{P}_{0}\right) \subseteq \boldsymbol{Z}(\mathfrak{P})$, there is an elementary subgroup $\mathfrak{F}$ of $\mathfrak{P}^{*}$ of order $p^{2}$ which is normal in $\mathfrak{P}$ and 
contains $\Omega_{1}\left(\mathfrak{P}^{*} \cap \mathfrak{P}_{0}\right)$. By Lemma 6.4, it follows that $\Omega_{1}\left(\mathfrak{P}^{*} \cap \mathfrak{P}_{0}\right)$ centralizes $\Re$, so again (*) holds. Suppose that $\mathfrak{P}^{*} \cap \boldsymbol{C}\left(\mathfrak{P}_{1}\right)$ is cyclic. Since $\mathfrak{P}^{*} \mathfrak{P}_{1}=\mathfrak{P}$ and $\mathfrak{P}_{0} \cap \mathfrak{P}_{1}=1$, it follows that $\mathscr{S} \mathscr{C} \mathscr{N}_{3}\left(\mathfrak{P}^{*}\right) \neq \varnothing$. Now since $\mathscr{S} \mathscr{C} \mathscr{N}_{2}\left(\mathfrak{B}_{1}\right) \neq \varnothing$, and since $\mathscr{S} \mathscr{C} \mathscr{N}_{3}\left(\mathfrak{P}_{0}\right) \neq \varnothing$, it follows that $\mathfrak{P}$ contains a normal elementary subgroup $\mathfrak{A}$ of order $p^{5}$. Let

$$
\mathfrak{A}^{*}=\mathfrak{P}^{*} \cap \mathfrak{A} \text {. }
$$

Thus, $\mathfrak{2}^{*}$ is of order precisely $p^{3}$, since $\mathfrak{P}^{*}$ contains a normal cyclic subgroup $\mathfrak{S}^{*} \cap \boldsymbol{C}\left(\mathfrak{P}_{1}\right)$ with factor group elementary of order $1, p$ or $p^{2}$. Since $\mathfrak{P} \subseteq N\left(\mathfrak{P}_{1}\right)^{\prime}$, it follows easily from 0.3.4 that $C\left(\mathfrak{P}_{1}\right) \cap \mathfrak{U}^{*}$ is noncyclic. This is impossible, so we conclude that

$$
\mathscr{S} \mathscr{C} \mathscr{N}_{3}\left(\mathfrak{P}_{1}\right) \neq \varnothing \text {. }
$$

Choose an element $\mathfrak{B}$ of $\mathscr{S} \mathscr{C} \mathscr{N}_{3}\left(\mathfrak{Q}_{1}\right)$ with $\mathfrak{B} \triangleleft \mathfrak{l}$. Suppose $\mathfrak{B}$ is faithfully represented on some element of $И\left(\mathfrak{B}^{*} ; 2\right)$, where

$$
\mathfrak{B}^{*} \in \mathscr{S} \mathscr{C} \mathfrak{N}(\mathfrak{\Omega})
$$

$\mathfrak{B} \subseteq \mathfrak{B}^{*}$. Let $\mathfrak{R}$ be the element of $И\left(\mathfrak{F}_{1} \mathfrak{Q}_{1} ; 2\right)$ given by Theorem 12.2(ii), with $\mathfrak{B} \mathfrak{\subseteq} N(\mathfrak{R})$. In this case, $\left[\mathfrak{P}_{0}, \mathfrak{D}_{1}\right]$ centralizes $\mathfrak{R}$, so

$$
\left[\mathfrak{F}_{0}, \mathfrak{Q}_{1}\right] \cap \mathfrak{B}=1 \text {. }
$$

This is impossible since $\mathfrak{B} \supseteq \boldsymbol{Z}\left(\mathfrak{\beth}_{1}\right)$. Thus, $\mathfrak{B}$ is not represented faithfully on any element of $И\left(\mathfrak{B}^{*} ; 2\right)$. We can thus choose $Z$ in $\boldsymbol{Z}(\mathfrak{\Omega}) \cap \mathfrak{\Omega}_{1}^{*}$ such that $Z$ centralizes a maximal element $\Re_{0}$ of $И(\Omega ; 2)$. Hence, $C(Z) \supseteqq\left\langle\mathfrak{P}_{1}, \Re_{0}\right\rangle$ and we assume without loss of generality that $\mathfrak{P}_{1} \Re_{0}$ is a group. It follows that $N\left(\mathfrak{P}_{1} \Re_{0}\right)$ contains a $S_{p q}$-subgroup of (S). By $C_{p, q}$, we can choose a maximal element $\Re_{1}=\Re_{0}^{G}$ of $И(\mathfrak{\Omega} ; 2)$ such that $\mathfrak{B \Omega}$ is permutable with $\Re_{1}$. Thus, $\left(^{*}\right)$ holds in this case, too.

We will next show that there are a prime $q$ and an elementary subgroup $\left[5\right.$ of $\Omega$ of order $q^{2}$ such that

(i) $\left(5\right.$ is normal in a $S_{q}$-subgroup of $\Re$.

(ii) 5 centralizes every element of $И(\Im ; 2)$.

(iii) $\quad[5 \subseteq F(\Re)$.

If $\mathfrak{P}_{0} \cap \boldsymbol{F}(\Re)$ is noncyclic, we may take $q=p$, so suppose that $\mathfrak{P}_{0} \cap \boldsymbol{F}(\Re)$ is cyclic. We can then choose $q$ in $\pi(\boldsymbol{F}(\Re)), q \neq p$ such that $\mathfrak{\beta}_{0}$ does not centralize the $S_{q}$-subgroup $\widetilde{F}_{q}$ of $\boldsymbol{F}(\Re)$. Let $\cong$ be a $S_{q}$-subgroup

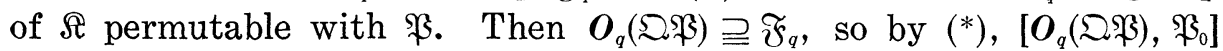
centralizes every element of $\boldsymbol{U}\left(\boldsymbol{O}_{q}(\mathfrak{\Im}) ; 2\right)$. Let $\mathfrak{\Omega}^{*}$ be the largest

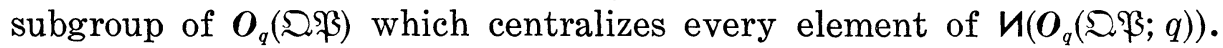

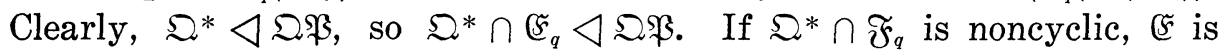


clearly available. Suppose $\mathfrak{Z}^{*} \cap \mathfrak{F}_{q}$ is cyclic. Then in particular, $\mathfrak{F}_{q} \cap\left[O_{q}(\mathfrak{N P}), \mathfrak{P}_{0}\right]$ is cyclic. We will show that this is not the case.

Let $\mathfrak{Q}=\boldsymbol{N}\left(\boldsymbol{O}_{q}(\mathfrak{Q})\right)$ and let $\tilde{\mathfrak{Q}}$ be the largest subgroup of $\boldsymbol{O}_{q}(\mathfrak{\Omega})$ which centralizes every element of $U(\mathfrak{\Omega} ; \sigma-\{q\})$. Let $\mathbb{L}=N(Z(\tilde{\mathfrak{\Omega}}))$. Then $\langle\mathbb{R}, \Re\rangle \subseteq \mathbb{R}^{*}$. By Corollary $6.2, \mathfrak{P} \subseteq \mathbb{R}^{\prime}$, so $\mathfrak{P} \subseteq \mathbb{R}^{* \prime}$. Since $\mathfrak{P}_{0}$ does not centralize $\mathfrak{F}_{q}$ by hypothesis, $\left[\mathfrak{\mho}_{q}, \mathfrak{F}_{0}\right]$ is noncyclic.

Let $(5$ satisfy (12.5.i), (12.5.ii) and (12.5.iii). It follows easily from the definition of $\pi$ that $(5$ centralizes every element of $U(\xi ; r)$ for all primes $r \neq 3, q$. Thus, $\mathbb{L}=\left\langle\mathfrak{S} \mid \mathfrak{F} \in U\left(\mathfrak{F} ; q^{\prime}\right)\right\rangle$ is a solvable $q^{\prime}$ group, and $\Re \subseteq N(\Omega)$. It follows that every element of $И(\Re)$ is contained in $N(\mathbb{R})=\mathfrak{M}$, say. Hence, $\mathscr{A}(p) \subseteq \mathscr{C l}^{*}(\mathbb{S})$, by Theorem 10.6. It then follows that every element of $U(\mathfrak{T})$ is contained in $\mathfrak{M}$, so $\mathfrak{I} \subseteq \mathfrak{M}$ and by Theorem 10.7, $N(\mathfrak{I}) \subseteq \mathfrak{M}, C(I) \subseteq \mathfrak{M}$ for all involutions $I$ of $\mathfrak{T}$. This is not the case. We have shown that Hypothesis 12.2 is not satisfied.

Let $\sigma$ be the set of all primes $q \geqq 5$ such that $\mathfrak{I}$ normalizes some nonidentity $q$-subgroup of (S).

Hypothesis 12.3. There is a prime $p \geqq 5$ such that $p \| \boldsymbol{A}_{\Theta}\left(\mathfrak{S}_{\mathfrak{C}}\right) \mid$ for some 2-subgroup $\mathfrak{S}$ of $\mathbb{S}$.

By Hypothesis 12.3 and Lemma 5.51, there is a normal subgroup $\mathfrak{S C}$ of $\mathfrak{I}$ such that $p \| \boldsymbol{A}_{\circledast}(\mathfrak{S}) \mid$. Let $\Omega=N(\mathfrak{K})$ and let $\Omega_{0}$ be a $S_{2}$-subgroup of $\Re$ which contains $\mathfrak{I}$. Let $\mathfrak{P}$ be a $S_{p}$-subgroup of $\mathfrak{R}_{0}$. Let $\mathfrak{S}_{0}=\boldsymbol{O}_{p_{2}}\left(\Re_{0}\right) \cap \mathfrak{I}, \mathfrak{P}_{0}=\mathfrak{P} \cap \boldsymbol{O}_{p_{2} p}\left(\mathfrak{R}_{0}\right)$. Thus, $\left[\mathfrak{S}_{0}, \mathfrak{P}_{0}\right]=\mathfrak{S}_{1} \triangleleft \mathfrak{I}$, and $\mathfrak{P}_{0}$ does not centralize $\mathfrak{S}_{0}$. If $q \in \sigma$ and $\mathfrak{Q}$ is a maximal element of $И(\mathfrak{T} ; q)$, then since neither Hypothesis 12.1 nor 12.2 is satisfied, it

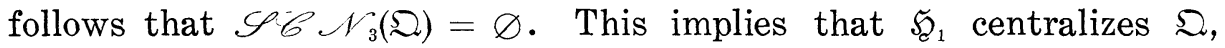
by Theorem 6.1. Since $p \geqq 5, \mathfrak{K}_{1}$ contains an element $\mathfrak{B}$ of $\mathscr{C}^{*}(\mathfrak{T})$. Thus, $\mathfrak{B}$ centralizes every element of $\mathcal{H}\left(\mathfrak{B} ;\{2,3\}^{\prime}\right)$. It follows that $\langle\mathfrak{X} \mid \mathfrak{U} \in \boldsymbol{U}(\mathfrak{T})\rangle$ is of odd order. This is not the case, so Hypothesis 12.3 is not satisfied.

Hypothesis 12.4. There is an element $\mathfrak{B}$ of $\mathscr{C}^{*}(\mathfrak{T})$ which centralizes every element of $И(\mathfrak{B} ; \sigma)$.

Suppose Hypothesis 12.4 is satisfied. Then $\langle\mathfrak{S} \mid \mathfrak{F} \in U(\mathfrak{T})\rangle$ is of odd order. Since this is not the case, Hypothesis 12.4 is not satisfied.

HYPOTHESIS 12.5. $\sigma \subseteq \pi_{1}$.

Suppose Hypothesis 12.5 is satisfied. Let $p$ be the largest prime in $\sigma$, and let $q \in \sigma$. Since $\mathscr{S} \mathscr{C} \mathscr{N}_{3}(\mathfrak{T}) \neq \varnothing$, we can choose an element $I$ of $\mathfrak{I}^{\sharp}$ which centralizes a $S_{q}$-subgroup of $\mathbb{B}$ and a $S_{p}$-subgroup 
of $\mathbb{B}$. Hence, if $\mathfrak{P}$ is a $S_{p}$-subgroup of $\mathbb{B}$ which is normalized by $\mathfrak{T}$, then $N(\mathfrak{P})$ contains a $S_{\sigma}$-subgroup $\mathbb{S}$ of $\mathbb{S}$ which is normalized by $\mathfrak{T}$. Also, $\mathfrak{T}^{\prime}$ centralizes $\mathfrak{S}$, so $\mathfrak{T}^{\prime}$ contains no element of $\mathscr{U}^{*}(\mathfrak{T})$. Hence, $\boldsymbol{Z}\left(\mathfrak{I}^{\prime}\right)$ is cyclic, so by a well known property of 2 -groups, $\mathfrak{I}^{\prime}$ is cyclic. Clearly, $\mathfrak{T}^{\prime} \neq 1$, since $\mathfrak{T}$ does not centralize $\mathfrak{S}$, while each Sylow subgroup of $\mathfrak{S}$ which is normalized by $\mathfrak{I}$ dominates $\mathfrak{T}$. If $\Omega_{1}\left(\mathfrak{I}^{\prime}\right)$ is weakly closed in $\mathfrak{I}$, then $\mathfrak{I} \subseteq N\left(\Omega_{1}\left(\mathfrak{I}^{\prime}\right)\right)^{\prime}$, so that $\mathfrak{I}$ centralizes $\mathfrak{S}$. Hence, $\Omega_{1}\left(\mathfrak{I}^{\prime}\right)$ is not weakly closed in $\mathfrak{I}$. Let $\Omega_{1}\left(\mathfrak{T}^{\prime}\right)=3=\langle Z\rangle$, and choose $G$ in $(S)$ so that $3^{G} \subset \mathfrak{I}, Z^{G}=Z_{1} \neq Z, 3^{G}=3_{1}$. We may assume that $3 \subseteq \mathfrak{T}^{G}$. Let $\xi$ be an elementary normal subgroup of $\mathfrak{T}^{G}$ of order 8 which contains $3_{1}$. Let $\mathfrak{\xi}_{0}=\boldsymbol{C}_{\mathbb{E}}(Z)$ so that $\left|\mathfrak{F}_{0}\right| \geqq 4$. We assume without loss of generality that $\mathfrak{F}_{0} \subseteq \mathfrak{I}$. Then $\mathfrak{F}_{0}$ normalizes $\mathfrak{S}$, but $3_{1}$ does not centralize $\mathfrak{S}$. We may choose $E$ in $\mathbb{F}_{0}^{*}$ so that $C_{\mathscr{\Im}}(E)$ is not centralized by $Z_{1}$. Since Hypothesis 12.3 is not satisfied, a $S_{\sigma, 2^{-}}$ subgroup of $\boldsymbol{C}(E)$ is $\sigma$-closed. Hence, there is an element $\widetilde{F}$ of $\mathscr{S} \mathscr{C} \mathscr{N}\left(\mathfrak{D}^{G}\right)$ which normalizes a $\sigma$-subgroup $\mathfrak{S}_{0}$ of (s) with $Z_{1} \notin \boldsymbol{C}\left(\mathfrak{S}_{0}\right)$. This is not possible, since $Z$ centralizes every element of $И(\mathfrak{T} ; \sigma)$. We have shown that Hypothesis 12.5 does not hold.

Let $\sigma_{i}=\sigma \cap \pi_{i}, i=1,2$. Since Hypotheses 12.1, 12.2, 12.3 fail,it follows that $\sigma=\sigma_{1} \cup \sigma_{2}$, and since Hypothesis 12.5 fails, $\sigma_{2} \neq \varnothing$. Let $p$ be the largest prime in $\sigma_{2}$, and let $\mathfrak{B}$ be a $S_{p}$-subgroup of (S). Let $₹$ be a normal elementary subgroup of $\mathfrak{P}$ of order $p^{2}$. Maximality of $p$ guarantees that for each $q$ in $\sigma_{2}-\{p\}$, 5 centralizes every element of $\boldsymbol{U}(\mathfrak{\xi} ; q)$. A routine argument shows that $(\xi 5$ centralizes every element of $\boldsymbol{U}\left(\mathfrak{s} ; \sigma_{1}\right)$. It follows that $\mathbb{5}$ centralizes every element of of $\boldsymbol{U}\left(\mathfrak{F} ;\{p, 3\}^{\prime}\right)$, so $\mathbb{L}=\langle\mathfrak{S} \mid \mathfrak{S} \in \boldsymbol{U}(\mathfrak{F})\rangle$ is a solvable $p^{\prime}$-group. Thus $\boldsymbol{N}(\mathbb{2}) \supseteqq \mathfrak{I}$, and it follows that $\langle\mathfrak{S} \mid \mathfrak{S C} \in \boldsymbol{U}(\mathfrak{T})\rangle$ is of odd order. Since this is not the case, we have derived the desired contradiction.

Received May 18, 1970. I am indebted to Professor J. H. Walter and Dr. Anne MacWilliams for pointing out several gaps. 


\title{
PACIFIC JOURNAL OF MATHEMATICS
}

\author{
EDITORS
}

\author{
H. SAMELSON \\ Stanford University \\ Stanford, California 94305

\section{R. HOBBY} \\ University of Washington \\ Seattle, Washington 98105
}

J. DUGUNDJI

Department of Mathematics

University of Southern California

Los Angeles, California 90007

RICHARD ARENS

University of California

Los Angeles, California 90024

\section{ASSOCIATE EDITORS}
E. F. BECKENBACH
B. H. NeUmanN
F. WoLF
K. YoshidA

\section{SUPPORTING INSTITUTIONS}

\author{
UNIVERSITY OF BRITISH COLUMBIA \\ CALIFORNIA INSTITUTE OF TECHNOLOGY \\ UNIVERSITY OF CALIFORNIA \\ MONTANA STATE UNIVERSITY \\ UNIVERSITY OF NEVADA \\ NEW MEXICO STATE UNIVERSITY \\ OREGON STATE UNIVERSITY \\ UNIVERSITY OF OREGON \\ OSAKA UNIVERSITY \\ UNIVERSITY OF SOUTHERN CALIFORNIA
}

\author{
STANFORD UNIVERSITY \\ UNIVERSITY OF TOKYO \\ UNIVERSITY OF UTAH \\ WASHINGTON STATE UNIVERSITY \\ UNIVERSITY OF WASHINGTON \\ $* * * *{ }^{*}$
AMERICAN MATHEMATICAL SOCIETY
CHEVRON RESEARCH CORPORATION
NAVAL WEAPONS CENTER
}

The Supporting Institutions listed above contribute to the cost of publication of this Journal, but they are not owners or publishers and have no responsibility for its content or policies.

Mathematical papers intended for publication in the Pacific Journal of Mathematics should be in typed form or offset-reproduced, (not dittoed), double spaced with large margins. Underline Greek letters in red, German in green, and script in blue. The first paragraph or two must be capable of being used separately as a synopsis of the entire paper. The editorial "we" must not be used in the synopsis, and items of the bibliography should not be cited there unless absolutely necessary, in which case they must be identified by author and Journal, rather than by item number. Manuscripts, in duplicate if possible, may be sent to any one of the four editors. Please classify according to the scheme of Math. Rev. Index to Vol. 39. All other communications to the editors should be addressed to the managing editor, Richard Arens, University of California, Los Angeles, California, 90024.

50 reprints are provided free for each article; additional copies may be obtained at cost in multiples of 50 .

The Pacific Journal of Mathematics is published monthly. Effective with Volume 16 the price per volume (3 numbers) is $\$ 8.00$; single issues, $\$ 3.00$. Special price for current issues to individual faculty members of supporting institutions and to individual members of the American Mathematical Society: $\$ 4.00$ per volume; single issues $\$ 1.50$. Back numbers are available.

Subscriptions, orders for back numbers, and changes of address should be sent to Pacific Journal of Mathematics, 103 Highland Boulevard, Berkeley, California, 94708.

PUBLISHED BY PACIFIC JOURNAL OF MATHEMATICS, A NON-PROFIT CORPORATION

Printed at Kokusai Bunken Insatsusha (International Academic Printing Co., Ltd.), 270, 3chome Totsuka-cho, Shinjuku-ku, Tokyo 160, Japan. 


\section{Pacific Journal of Mathematics}

Vol. 39, No. $2 \quad$ June, 1971

Edward Arthur Bertram, Permutations as products of conjugate infinite

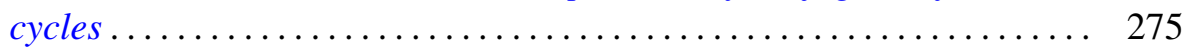

David Blair, Almost contact manifolds with Killing structure tensors ...... 285

Bruce Donald Calvert, Nonlinear equations of evolution ............. 293

Bohumil Cenkl and Giuliano Sorani, Cohomology groups associated with

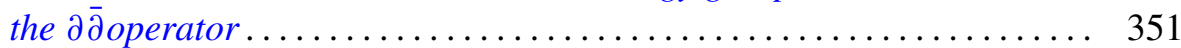

Martin Aaron Golubitsky and Bruce Lee Rothschild, Primitive subalgebras

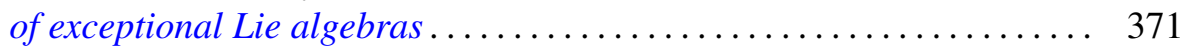

Thomas J. Jech, Two remarks on elementary embeddings of the universe ... 395

Harold H. Johnson, Conditions for isomorphism in partial differential equations........................................ 401

Solomon Leader, Measures on semilattices ..................... 407

Donald Steven Passman, Group rings satisfying a polynomial identity. II .......................................... 425

Ralph Tyrrell Rockafellar, Integrals which are convex functionals. II . . . . . 439

Stanisław Sławomir Świerczkowski, Cohomology of group germs and Lie

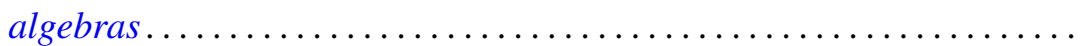

John Griggs Thompson, Nonsolvable finite groups all of whose local subgroups are solvable. III ............................ 483

Alan Curtiss Tucker, Matrix characterizations of circular-arc graphs .... 535 